\title{
Hadron potentials within the gauge/string correspondence
}

\author{
Frédéric Jugeau ${ }^{1,2}$ \\ 1 Institute of High Energy Physics, Chinese Academy of Sciences, \\ P. O. Box 918(4), Beijing 100049, China \\ 2 Theoretical Physics Center for Science Facilities, Chinese Academy of Sciences, \\ Beijing 100049, China \\ jugeau@ihep.ac.cn
}

\begin{abstract}
It is known, since the 70 's, that the large $N$ 't Hooft limit of gauge theories is related to string theories. In 1998, J. M. Maldacena identified precisely such a relation: the so-called $A d S / \mathrm{CFT}$ correspondence which speculates a duality between a large $N$ strongly-coupled supersymmetric and conformal Yang-Mills theory in four dimensions and a weakly-coupled string theory defined in a five-dimensional anti-de Sitter $A d S_{5}$ space-time. This review aims at introducing concepts and methods used to derive, in the framework of the gauge/string correspondence, the interaction potentials of mesons and baryons at zero and finite temperature. The dual string configurations associated with the different kinds of hadrons are described and their behaviours at short and large distances are understood. Although the application of Maldacena's AdS/CFT conjecture to QCD is not straightforward, QCD being neither supersymmetric nor conformal, the $A d S / \mathrm{QCD}$ correspondence approach attempts to identify the dual theory of QCD. Especially, the study of heavy quark-antiquark bound-states leads to establish general dual criteria for the confinement.
\end{abstract}

PACS numbers: 11.25.Tq, 11.25.-w, 12.38.Aw, 12.38.Lg, 12.40.Yx.

Keywords: AdS/CFT correspondence, holographic models of QCD, Wilson loop, hadron potentials, confinement. 


\section{Contents}

1 Introduction $\quad 3$

2 The static potential in QCD $\quad 9$

3 The heavy quark potential in the $A d S / C F T$ correspondence 14

4 The static potential at finite temperature $\quad 20$

4.1 The conformal behaviour of the Wilson loop at finite temperature . . . . . 20

4.2 The area law in three-dimensional Yang-Mills theory . . . . . . . . . . . . 24

4.3 The area law in four-dimensional Yang-Mills theory . . . . . . . . . . . . . 29

5 The heavy quark potential in holographic models of QCD 31

5.1 Andreev and Zakharov's model . . . . . . . . . . . . . . . . . . . 31

5.1.1 The heavy quark potential at large distances . . . . . . . . . . . . 33

5.1.2 The heavy quark potential at short distances . . . . . . . . . . . . 34

5.2 The heavy quark potential from general geometry in $A d S / \mathrm{QCD}$. . . . . . 36

$6 \quad$ The supergravity description of baryons 39

6.1 The baryon potential within the $A d S / C F T$ correspondence . . . . . . . . . 39

6.2 Existence of $A d S / C F T$ baryons made of $k<N$ quarks . . . . . . . . . . . 42

6.3 Baryons in three-dimensional Yang-Mills theory . . . . . . . . . . . . . . 44

$\begin{array}{lll}7 & \text { Conclusion } & 45\end{array}$

A Brief review of the Wilson loop in QCD 46

A.1 The gauge line. . . . . . . . . . . . . . . . . . . 47

A.2 Mandelstam's formula . . . . . . . . . . . . . . . . . . . 49

A.3 The equations of QCD . . . . . . . . . . . . . . . . 52

A.4 The Wilson loop: the colour trace of a closed gauge line . . . . . . . . . . . 53

A.5 The Migdal-Makeenko equation and the loop equation . . . . . . . . . . 53

A.6 The renormalization of the Wilson loop . . . . . . . . . . . . . . . 58

A.7 The Wilson loop in $\mathrm{QCD}_{2} \quad \ldots \ldots \ldots$. . . . . . . . . . 59 


\section{Introduction}

A crucial breakthrough in the attempt to deal with strongly-coupled Yang-Mills theories came with the $A d S / C F T$ correspondence, proposed by J. M. Maldacena in 1998 [1], that conjectures a duality between the supergravity approximation of a superstring/M-theory living in a $d$-dimensional anti-de Sitter space $\left(A d S_{d}\right)$ times a compact manifold and the 't Hooft limit of a maximally $\mathcal{N}=4$ superconformal $S U(N)$ gauge theory defined on the $(d-1)$-dimensional boundary space $\left(\partial A d S_{d}\right)$. Shortly afterwards, $[2,3]$ established the method for deriving conformal dimensions of operators and correlators in conformal field theory by means of dual superstring theory. In addition, J. M. Maldacena considered the problem of calculating expectation value of Wilson loop [4]. This issue is of particular importance since the Wilson loop, through the area law, consists of one of the most efficient tools for probing the large distance properties of confining QCD-like gauge theories. The purpose of the present paper is thus to review, within the AdS/CFT and the $A d S / \mathrm{QCD}$ correspondences, the short and large distance behaviours of the Wilson loop in the conformal and non-conformal cases, and its use for deriving the interaction potentials of bound-states of quarks.

First of all, let us motivate the gauge/string correspondence which arises from low energy arguments when one considers energies $E$ much smaller than the energy scale associated with the typical string length $\ell_{s}=\sqrt{\alpha^{\prime}}$ :

$$
E \ll \frac{1}{\sqrt{\alpha^{\prime}}} .
$$

As a matter of fact, we will be mostly interested, in the sequel, in a stack of $N$ coincident D3-branes (A D p-brane is an extended physical object with $p$ spatial dimensions. The capital letter D in D $p$-brane stands for Dirichlet as the coordinates of the open string endpoints normal to the brane must satisfy Dirichlet boundary conditions). Therefore, it is worthwhile to recall briefly some results of the open string spectroscopy in the presence of $\mathrm{D} p$-branes. In a $d$-dimensional flat space-time, the (square) mass spectrum of an open string of which the endpoints lie on a D $p$-brane reads

$$
M^{2}=\frac{1}{\alpha^{\prime}}\left(N^{(i)}+N^{(a)}-1\right)
$$

where $N^{(i)} \equiv \sum_{n=1}^{\infty} \sum_{i=2}^{p} n a_{n}^{i \dagger} a_{n}^{i}$ and $N^{(a)} \equiv \sum_{m=1}^{\infty} \sum_{a=p+1}^{d} m a_{m}^{a \dagger} a_{m}^{a}$, expressed in terms of creation and annihilation operators, count the number of modes along the directions, respectively tangential $(i=2, \ldots, p)$ and normal $(a=p+1, \ldots, d)$ to the $\mathrm{D} p$-brane. The low energy limit (1.1) can then be understood as follows. Whereas the energies are kept 
bounded (and the closed string coupling constant $g_{s}$ is kept fixed) the typical length scale of the string is put to zero [1]:

$$
\alpha^{\prime} \rightarrow 0 \quad\left(E \text { bounded and } g_{s} \text { fixed }\right) \text {. }
$$

Hence, the massive modes of the open string decouple and only remain the massless states. For this reason, the low energy limit is also called the decoupling limit. The massless modes are of two types. The first kind of massless states consists of the oscillators $a_{m}^{a}{ }^{\dagger}$ 's normal to the brane acting on the (tachyonic $M^{2}=-\frac{1}{\alpha^{\prime}}$ ) ground-states $|\Omega\rangle: a_{m}^{a}{ }^{\dagger}|\Omega\rangle$. Since the index $a$ lives in the space normal to the brane, it is not a Lorentz index for the brane and the $(d-p)$ corresponding states are massless scalar states (these states represent slight parallel displacements of the brane. In fact, a space-filling brane does not have massless scalar excitation because it has precisely nowhere to turn). More interesting for us are the oscillators $a_{n}^{i \dagger}$ 's tangent to the brane. Since the index $i$ lives on the brane, they give rise to $(p+1)-2$ massless vector states. When $p=3$, one recognizes the two polarization states of the photon field living in the four-dimensional world-volume of the D3-brane. What happens with a stack of $N$ coincident D3-branes is the following. We know that a gauge field lives on each of the branes. But now, the open strings are allowed to pass from a brane to another without requiring additional amount of energy (contrary to an open string stretched out between two parallel non-coincident D $p$-branes where the mass-squared gains then an extra positive contribution stemming from the classical stretching energy (3.80)). Since the branes are distinguishable, the orientations $[\alpha \beta]$ and $[\beta \alpha]$ of an open string from the brane $\alpha$ to the brane $\beta$ for instance are not equivalent (the indices $\alpha, \beta=1, \ldots, N$ are called Chan-Paton indices). Therefore, with the $N$ gauge fields associated with the $N$ D3-branes, we have altogether $N+N(N-1)=N^{2}$ selfinteracting gauge fields living in the $(\mathrm{p}+1=4)$-dimensional world-volume of the stack. In other words, an $U(N)$ Yang-Mills theory in the Minkowski space-time. As a matter of fact, the previous statement is not rigorously true: all the gauge fields do not interact with each other. Provided that we perform a change of basis in the space of states, a noninteracting $U(1)$ gauge field can be identified which carries indeed a zero charge as seen by the other eight self-interacting gauge fields which define, in turn, a $S U(N)$ Yang-Mills theory.

We are now ready to establish the $A d S / C F T$ correspondence [1, 5]. First of all, let us consider the $N$ coincident D3-branes described above plunged into a ten-dimensional flat space-time (the bulk with $d=10$ ). Although the branes carry charge and mass, their back-reaction on the bulk is neglected here and they are just considered as boundary conditions for the open strings. At low energies, the four-dimensional physics is described by a maximally $\mathcal{N}=4 S U(N)$ supersymmetric Yang-Mills theory. Such a theory is also invariant under the conformal $S O(2,4)$ group, i.e. does not contain any length scale. 
The number of generators in this conformal field theory (or CFT) is $\operatorname{dim}(S O(2, n))=$ $\operatorname{dim}(S O(n+2))=\frac{(n+2)(n+1)}{2} \underset{(n=4)}{=} 15$, which is also the dimension of the isometry group of the anti-de Sitter space-time $A d S_{5}$ : here is a first insight towards the establishment of the $A d S / C F T$ correspondence conjecture. As for the closed strings, of which the massless spectrum contains the graviton states ${ }^{1}$, they are allowed to propagate throughout the bulk. The strength of the gravitational interactions is governed by Newton's constant which, at ten dimensions, is given by $G_{10}=8 \pi^{6} g_{s}^{2} \alpha^{\prime 4}$ (we work in natural units where $\hbar=c=1$ ). In the decoupling limit where $\alpha^{\prime} \rightarrow 0$ with $g_{s}$ fixed, Newton's constant vanishes and the interactions in gravity become free in the infrared (IR): the closed strings do not interact with each other anymore but also with the $S U(N)$ gauge fields living on the branes since gravity couples universally to all forms of matter. Hence, we are left with a $S U(N)$ super-Yang-Mills theory defined in the four-dimensional Minkowski world-volume of the $N$ coincident D3-branes and a decoupled set of non-interacting closed strings in ten-dimensional flat space-time.

In the second viewpoint, the $N$ D3-branes are solution of the gravitational field equations of a ten-dimensional type IIB (oriented closed) superstring theory. Since the branes carry energy, they warp locally space-time geometry: a throat develops in the depths of which are the branes while the space-time infinitely far away the throat is asymptotically flat. This throat can be visualized as an infinitely deep cylinder, the radius $R$ of which becoming constant (this so-called radius of the horizon should not be confused with the horizon of a Schwarzschild black hole since the geometry of the latter is quite different from the throat geometry). Moreover, since the D3-branes define a four-dimensional space-time on their world-volume, they appear as a point source of energy with respect to the remaining six space-like dimensions. Precisely, six coordinates are required in order to define a compact 5-sphere $S^{5}$ and, thus, the point-like throat is surrounded, in the space transverse to the branes, by concentric $S^{5}$ 's. As we move near the throat, the volume of the 5 -spheres tends to a constant with also $R$ as the radius. Let us write down the geometry of the $N$ coincident (extremal) D3-branes [6]:

$$
d s^{2}=\left(1+\frac{R^{4}}{\alpha^{\prime 4} u^{4}}\right)^{-\frac{1}{2}} \eta_{\mu \nu} d x^{\mu} d x^{\nu}+\left(1+\frac{R^{4}}{\alpha^{\prime 4} u^{4}}\right)^{\frac{1}{2}}\left(\alpha^{\prime 2} d u^{2}+\alpha^{\prime 2} u^{2} d \Omega_{5}^{2}\right)
$$

where $\eta_{\mu \nu}=\operatorname{diag}(-1,+1,+1,+1)$ is the Minkowski metric tensor of the D3-brane world-

\footnotetext{
${ }^{1}$ The square masses of the closed strings are:

$$
M^{2}=\frac{2}{\alpha^{\prime}}(N+\bar{N}-2)
$$

with $N=\sum_{n=1}^{\infty} \sum_{I=2}^{d} n a_{n}^{I^{\dagger}} a_{n}^{I}$ and $\bar{N}=\sum_{n=1}^{\infty} \sum_{I=2}^{d} n \bar{a}_{n}^{I^{\dagger}} \bar{a}_{n}^{I}$ the number operators of respectively the left- and right-moving modes which satisfy the constraint $N=\bar{N}$.
} 
volume $(\mu, \nu=0, \ldots, 3)$ and $d \Omega_{5}^{2}$ is the line element of the unit sphere $S^{5}$. The D3-branes are located infinitely deep in the throat $\alpha^{\prime} u \rightarrow 0$. In the $A d S / C F T$ correspondence, the "holographic" radial coordinate $\alpha^{\prime} u$ is dual to the energy scale under which is observed the $S U(N)$ super-Yang-Mills theory ( $u$ has indeed the dimension of an energy). Being the $N$ D3-branes and the horizon deep down the throat, they can never be reached. Consequently, it appears two decoupled regions: the asymptotically flat space-time far away at infinity $\left(\alpha^{\prime} u \rightarrow+\infty\right)$ and the region near the horizon $\left(\alpha^{\prime} u \rightarrow 0\right)$. Near the horizon, the metric (1.5) becomes:

$$
d s^{2}=\alpha^{\prime}\left(\frac{\alpha^{\prime} u^{2}}{R^{2}} \eta_{\mu \nu} d x^{\mu} d x^{\nu}+\frac{R^{2}}{\alpha^{\prime}} \frac{d u^{2}}{u^{2}}\right)+R^{2} d \Omega_{5}^{2}
$$

which is nothing else than the $A d S_{5} \times S^{5}$ geometry. There is an overall $\alpha^{\prime}$ factor, so the metric remains constant in $\alpha^{\prime}$ units. The near-horizon approximation (1.6) could have also been obtained from the decoupling limit (1.3) as defined by [1]: whereas $\alpha^{\prime} \rightarrow 0$, we keep the energies bounded which is indeed consistent with the fact that the energy scale $u$ remains constant when passing from (1.5) to (1.6). As seen by an observer living in the asymptotically flat Minkowski space-time, two distinctive low energy physics arise associated with the two decoupled space-time regions. On the one hand, there is obviously the low energy physics which rules near the observer. Without brane where attached open string endpoints, it consists, according to the decoupling limit, of massless modes of noninteracting closed strings. On the other hand, near the horizon, any finite energy mode which attempts to reach the observer need to overcome the gravitational well generated by the throat. These modes are then perceived as massless by the observer and deeper they are in the throat, higher can be their energies. As a result, the whole tower of massive modes has to be taken into account by the observer at asymptotic infinity. So, in the second viewpoint, we have a type IIB closed superstring theory living, near the horizon, in a ten-dimensional $A d S_{5} \times S^{5}$ space-time and, once again, a decoupled set of non-interacting closed strings in ten-dimensional flat space-time. We see that the two descriptions share the same decoupled system, namely, the set of non-interacting closed strings. We are thus led to conjecture that, at low energies, a $S U(N)$ super-Yang-Mills theory living in a four-dimensional Minkowski space-time and a type IIB closed superstring theory on $A d S_{5} \times S^{5}$ describe the same physics. This is the $A d S / C F T$ correspondence [1].

As a matter of fact, although these two very different theoretical frameworks are believed (and checked, see for instance [7]) to be dual to each other, their tractability domains turn out to be opposite. To see that, let us focus on the relations involving the two sets of parameters $\left(R, g_{s}\right)$ and $\left(N, g_{Y M}\right)$ of the dual theories $\left(g_{Y M}\right.$ is the Yang-Mills coupling constant). The radius of the horizon $R$ (the so-called $A d S$ radius) is given in 
terms of the string length scale $\alpha^{\prime}$ by

$$
\frac{R^{2}}{\alpha^{\prime}} \propto \sqrt{g_{s} N}
$$

while

$$
g_{s} \propto g_{Y M}^{2} .
$$

According to (1.8), the ratio (1.7) implies the so-called 't Hooft coupling constant:

$$
\lambda \equiv g_{Y M}^{2} N
$$

which is the relevant coupling constant when considering gauge theory in the large $N$ limit. These two duality relations can be derived, for the former, from general considerations on $\mathrm{D} p$-branes involving their number, their tension and Newton's constant, and for the latter, by considering the weak field expansion of the Dirac-Born-Infeld action (6.208) which governs the dynamics of $\mathrm{D} p$-branes carrying electromagnetic fields on their world-volumes. Especially, the second relation tells us that to a weak string coupling constant $g_{s} \ll 1$ corresponds a weak Yang-Mills coupling constant $g_{Y M} \ll 1$, which seems convenient since it is easier to deal with any theory in the perturbative regime. Thus, the $A d S / C F T$ correspondence seems straightforward to check. Nevertheless, let us consider the case where $N$ is finite. The first relation implies then $R^{2} \ll \alpha^{\prime}$ i.e. the $A d S_{5}$ scalar curvature (proportional to $1 / R^{2}$ ) is much larger than $1 / \alpha^{\prime 2}$. As a result, all the stringy effects have to be considered but we do not know yet how to solve tree-level superstring theory on $A d S_{5} \times S^{5}$. So, let us see what happens in the large $N$ limit when the 't Hooft coupling $\lambda$ is also large. In this case $R^{2} \gg \alpha^{\prime}$ and the strings appear as point-like particles. The ten-dimensional free superstring theory can then be approximated by its low energy effective theory : a tractable ten-dimensional supergravity theory where the stringy effects are corrections of order $O\left(\alpha^{\prime 3}\right)$. On the other side of the duality, we have a $S U(N)$ super-Yang-Mills theory which is now strongly-coupled. Although QCD is neither supersymmetric nor conformal, one sees the great interest in establishing a gauge/string correspondence for such a confining gauge theory. We already know that we have to break properly the underlying supersymmetry nature of the $A d S / \mathrm{CFT}$ correspondence and to deform the ten-dimensional $A d S_{5} \times S^{5}$ holographic space-time since QCD has a mass gap and is asymptotically conformal only at high energy (namely at $\alpha^{\prime} u \rightarrow+\infty$ in (1.5)). The strong coupling regime could then be investigated from a higher-dimensional dual supergravity theory (in the large $N$ limit with $N$ identified to the number of colours $N_{c}$ ). In practice, two complementary approaches exist which aim at identifying the dual theory of QCD. In the so-called top-down approach, the $A d S$ geometry is deformed into a Schwarzschild black hole- $A d S$ geometry where the horizon plays the role of an IR brane, the location of which being given in terms of the Beckenstein-Hawking temperature (i.e. 
the thermal temperature of the gauge theory) [8]. On the other hand, according to the more phenomenological bottom-up approach (or, roughly speaking, in the $A d S / \mathrm{QCD}$ correspondence though such a duality has not been established yet), five-dimensional holographic models which attempt to reproduce the main properties of QCD have been proposed. In the IR Hard Wall Model [9, 10, 11], a confining gauge theory can be obtained considering a truncated $A d S_{5}$ holographic space-time, the typical size of which representing the IR cutoff associated to the QCD mass gap. The light hadron spectroscopy [12], the meson and nucleon form factors [13], the two-point correlation functions [14], the deep inelastic scattering structure functions [15], the chiral symmetry breaking mechanism and the axial $U(1)$ anomaly [16] have been investigated. In particular, it has been showed that the spectrum satisfies a Kaluza-Klein behaviour $m_{n}^{2} \sim n^{2}$ instead of the expected Regge behaviour $m_{n}^{2} \sim n$ ( $n$ is the radial excitation number). To remedy this shortcoming, the IR Soft Wall Model has been proposed which consists in inserting a dilaton field in the $A d S_{5}$ space-time [17]. The phenomenological outcomes of this model have also been largely studied $[18,19]$.

The review is organized as follows: the main steps leading to the confining linear static potential of a heavy quark-antiquark bound-state through the four-point Green function and the area law of the Wilson loop in QCD are described in the section 2. The section 3 is devoted to Maldacena's calculation of the heavy quark potential within the $A d S / \mathrm{CFT}$ correspondence. Especially, the underlying conformal invariance and the intrinsically nonperturbative nature of the result is pointed out. In the section 4 , we consider the finite temperature version of the gauge/string correspondence as described by E. Witten [8] and derive, in three and four dimensions, linear quark-antiquark potentials of which the string tensions depend on the temperature. The Lüscher correction term at large distance is also discussed. The section 5 focuses on holographic models of QCD for which general dual criteria for the confinement can be established considering the bulk dynamics of dual string world-sheet. In the section 6 , we review the string/brane configuration dual to the baryons and stress, in particular, the existence of $A d S / \mathrm{CFT}$ "reduced" baryons made of $k<N$ quarks. At the end of the review, an appendix is devoted to the properties of the Wilson loop and to the loop space formalism of QCD. 


\section{The static potential in QCD}

The static potential between an infinitely massive quark and antiquark has been computed for a long time. The two-loop perturbative calculation gives [20]:

$$
V(r)=-C_{F} \frac{\alpha(r)}{r}
$$

with

$$
\begin{aligned}
\alpha(r)= & \alpha_{s}\left\{1+\left(a_{1}+\beta_{0} L\right) \frac{\alpha_{s}}{4 \pi}\right. \\
& \left.+\left[a_{2}+\beta_{0}^{2}\left(L^{2}+\frac{\pi^{2}}{3}\right)+\left(\beta_{1}+2 \beta_{0} a_{1}\right) L\right]\left(\frac{\alpha_{s}}{4 \pi}\right)^{2}+\ldots\right\} .
\end{aligned}
$$

$r$ is the distance between the quark and the antiquark and the (renormalization scale $\mu$-dependent) strong coupling constant $\alpha_{s}$ is in the modified minimal subtraction scheme $\overline{M S}: \quad \alpha_{s} \equiv \alpha_{s}^{(\overline{M S})}\left(\mu^{2}\right)$. We have defined $L \equiv 2 \gamma_{E}+\ln \left(\mu^{2} r^{2}\right)$ with $\gamma_{E} \simeq 0.5772$ the Euler-Mascheroni constant. The one-loop and two-loop constants $a_{1}$ and $a_{2}$ write out respectively:

$$
\begin{aligned}
a_{1}= & \frac{31}{9} C_{A}-\frac{20}{9} T_{F} n_{F} \\
a_{2}= & \left(\frac{4343}{162}+4 \pi^{2}-\frac{\pi^{2}}{4}+\frac{22}{3} \zeta(3)\right) C_{A}^{2}-\left(\frac{1789}{81}+\frac{56}{3} \zeta(3)\right) C_{A} T_{F} n_{F} \\
& -\left(\frac{55}{3}-16 \zeta(3)\right) C_{F} T_{F} n_{F}+\left(\frac{20}{9} T_{F} n_{F}\right)^{2}
\end{aligned}
$$

where $\zeta(s)$ is the Riemann zeta function and $n_{F}$ the number of massless quarks. In (2.11), the two first regularization scheme-independent coefficients of the $\beta$-function are:

$$
\begin{aligned}
& \beta_{0}=\frac{11}{3} C_{A}-\frac{4}{3} T_{F} n_{F}, \\
& \beta_{1}=\frac{34}{3} C_{A}^{2}-4 C_{F} T_{F} n_{F}-\frac{20}{3} C_{A} T_{F} n_{F} .
\end{aligned}
$$

$C_{F}$ and $C_{A}$ are the values of the Casimir operators in the fundamental and the adjoint representations respectively $\left(T^{a} T^{a}=C_{F} \mathbb{I}_{N_{c}}\right.$ with $T^{a}=\frac{\lambda^{a}}{2}$ the Gell-Mann matrices and $f^{a i j} f^{b i j}=C_{A} \delta^{a b}$ with $T_{i j}^{a}=-i f^{a i j}$ the structure constants). The trace normalization is $\operatorname{Tr}\left(T^{a} T^{b}\right)=T_{F} \delta^{a b}$ and, in the case of the colour gauge group $S U(3)_{c}$ of QCD, the colour factors become:

$$
\begin{aligned}
T_{F} & =\frac{1}{2}, \\
C_{F} & =T_{F}\left(\frac{N_{c}^{2}-1}{N_{c}}\right)=\frac{4}{3}, \\
C_{A} & =N_{c}=3 .
\end{aligned}
$$


Especially, at the lowest perturbative order, one recovers the one-gluon exchange contribution which has a Coulomb-like form:

$$
V(r)=-\frac{4}{3} \frac{\alpha_{s}}{r}
$$

More phenomenologically, E. Eichten, K. Gottfried, T. Kinoshita, K. D. Lane and T.M. Yan postulated, in the late 70s, that "many of the gross features of the charmonium $c \bar{c}$ potential can be simulated by the potential" (several phenomenological forms of the static potential have been proposed. See, for instance, the report [22]):

$$
V(r)=-\frac{\kappa}{r}+\sigma r+C
$$

"This is chosen to give a simple interpolation between the known Coulomb-like force at short distance and a linear growth of the static potential" [21]. In the so-called Cornell potential (2.20), $\kappa$ represents the coulomb strength, $\sigma$ the string tension and the constant $C$ fixes the origin of the potential. They are regarded as free parameters to be fitted on the spectrum. On the other hand, when one attempts to derive the potential on the lattice [23], a non-physical term in $1 / r^{2}$ is also often added to (2.20) in order to enhance the fit to data by simulating, for instance, running coupling effects.

The confining linear potential can also be extracted, in the static approximation, from the four-point, or two-particle (meson), Green function. The formalism involves the Wilson loop, a gauge invariant functional, which provides a physical observable able to measure the heavy quark interaction potential (see Eq.(2.47) below). The gauge invariant state of a quarkonium, bound-state of a quark and an antiquark, is defined by means of the so-called gauge line $U(y, x, \mathcal{C})$ :

$$
U(y, x ; \mathcal{C}) \equiv P e^{-i g \int_{x}^{y} A_{\mu}(x) d x^{\mu}}
$$

which carries out the parallel displacement of the gauge transformation from the point $x$ to the point $y$ along the curve $\mathcal{C}$ :

$$
|\phi(y, x)\rangle=\phi(y, x)|0\rangle=\bar{q}(y) U(y, x, \mathcal{C}) q(x)|0\rangle .
$$

The corresponding state, hermitian conjugate of (2.22), is:

$$
\langle\phi(y, x)|=\langle 0| \phi^{\dagger}(y, x)=\langle 0| \bar{q}(x) U\left(x, y, \mathcal{C}^{\prime}\right) q(y)
$$

where $\mathcal{C}$ and $\mathcal{C}^{\prime}$ describe the same space-time curve but oriented in opposite directions. Let us then consider the evolution amplitude of this quark-antiquark state during the lapse of time $T$. It consists of a four-point gauge invariant and colour-singlet Green function:

$$
\begin{aligned}
G^{(4)}\left(x_{1}, x_{2}, x_{1}^{\prime}, x_{2}^{\prime}\right) & \equiv \frac{1}{N_{c}}\left\langle\phi\left(x_{1}, x_{2}\right) \mid \phi\left(x_{1}^{\prime}, x_{2}^{\prime}\right)\right\rangle \\
& \equiv \frac{1}{N_{c}}\left\langle\mathcal{T}\left[\bar{q}\left(x_{2}\right) U\left(x_{2}, x_{1}\right) q\left(x_{1}\right) \bar{q}\left(x_{1}^{\prime}\right) U\left(x_{1}^{\prime}, x_{2}^{\prime}\right) q\left(x_{2}^{\prime}\right)\right]\right\rangle_{A, q, \bar{q}},
\end{aligned}
$$


the averaging being defined in the path-integral formalism. The dependence of the two gauge lines $U\left(x_{2}, x_{1}\right)$ and $U\left(x_{1}^{\prime}, x_{2}^{\prime}\right)$ on the curves $\mathcal{C}_{\left[x_{2} x_{1}\right]}$ and $\mathcal{C}_{\left[x_{1}^{\prime} x_{2}^{\prime}\right]}$ is implicit and the ordering prescription in (2.24) is the usual one defined by the chronological operator $\mathcal{T}$ :

$$
\mathcal{T}[q(x) \bar{q}(y)]=\theta\left(x_{0}-y_{0}\right) q(x) \bar{q}(y)-\theta\left(y_{0}-x_{0}\right) \bar{q}(y) q(x)
$$

The four-point Green function is normalized, in the large $N_{c}$ limit, by a factor $1 / N_{c}$. Since the action of QCD is quadratic in the fermion fields, the path-integral over the quarks is gaussian and gives according to the Wick theorem:

$G^{(4)}\left(x_{1}, x_{2}, x_{1}^{\prime}, x_{2}^{\prime}\right)=-\frac{1}{N_{c}}\left\langle\operatorname{det}\left(i \gamma^{\mu} D_{\mu}-m_{q}\right) U\left(x_{2}, x_{1}\right) S_{1}\left(x_{1}, x_{1}^{\prime} ; A_{\mu}\right) U\left(x_{1}^{\prime}, x_{2}^{\prime}\right) S_{2}\left(x_{2}^{\prime}, x_{2} ; A_{\mu}\right)\right\rangle_{A}$

were $D_{\mu}$ is the covariant derivative and $m_{q}$ the quark mass. It is usual to neglect, in the quenched approximation, the fermionic determinant giving rise, in perturbation theory, to quark-antiquark loops. In the large $N_{c}$ limit, this approximation is exact and we have:

$$
\operatorname{det}\left(i \gamma^{\mu} D_{\mu}-m_{q}\right)=1
$$

Moreover, (2.26) does not account for the other contribution associated to the $q \bar{q}$ annihilation process, only possible if $q\left(x_{1}\right)$ and $\bar{q}\left(x_{2}\right)$ (respectively $q\left(x_{2}^{\prime}\right)$ and $\bar{q}\left(x_{1}^{\prime}\right)$ ) have the same flavor.

In order to derive the static potential, Brown and Weisberger [24] wrote (2.26) in terms of the static quark and antiquark propagators in the presence of the external gluon field $A_{0}$ (formally, the static approximation consists in neglecting, in the equations of motion of the propagators, the spatial components of the covariant derivatives with respect to the temporal ones):

$$
\begin{aligned}
& S_{1 \text { stat. }}\left(x_{1}, x_{1}^{\prime} ; A_{0}\right)=S_{1}^{(0)}\left(x_{1}-x_{1}^{\prime}\right) U\left(x_{1}, x_{1}^{\prime}\right) \\
& S_{2 \text { stat. }}\left(x_{2}^{\prime}, x_{2} ; A_{0}\right)=S_{2}^{(0)}\left(x_{2}^{\prime}-x_{2}\right) U\left(x_{2}^{\prime}, x_{2}\right)
\end{aligned}
$$

were $S_{1}^{(0)}\left(x_{1}-x_{1}^{\prime}\right)$ and $S_{2}^{(0)}\left(x_{2}^{\prime}-x_{2}\right)$ are the free static propagators (namely, in the absence of gluon) which verify the following equations of motion:

$$
\begin{aligned}
\left(i \gamma_{1}^{0} \partial_{10}-m_{1}\right) S_{1}^{(0)}\left(x_{1}-x_{1}^{\prime}\right) & =i \delta^{4}\left(x_{1}-x_{1}^{\prime}\right) \\
S_{2}^{(0)}\left(x_{2}^{\prime}-x_{2}\right)\left(-i \gamma_{2}^{0} \overleftarrow{\partial}_{20}-m_{2}\right) & =i \delta^{4}\left(x_{2}^{\prime}-x_{2}\right)
\end{aligned}
$$

The expressions (2.28) and (2.29) allow one to write the static evolution amplitude into the form:

$$
G_{\text {stat. }}^{(4)}\left(x_{1}, x_{2}, x_{1}^{\prime}, x_{2}^{\prime}\right)=-\left\langle S_{1}^{(0)}\left(x_{1}-x_{1}^{\prime}\right) S_{2}^{(0)}\left(x_{2}^{\prime}-x_{2}\right) \frac{1}{N_{c}} \operatorname{Tr}_{c} U\left(x_{2}, x_{1}\right) U\left(x_{1}, x_{1}^{\prime}\right) U\left(x_{1}^{\prime}, x_{2}^{\prime}\right) U\left(x_{2}^{\prime}, x_{2}\right)\right\rangle_{A} .
$$


Then, the path-ordered product of the four gauge lines generates the trace on the colour space of a closed gauge line which is nothing else than the Wilson loop:

$$
\phi(\mathcal{C}) \equiv \operatorname{Tr}_{c} U\left(x_{1}, x_{1}, \mathcal{C}\right)=\operatorname{Tr}_{c} P e^{-i g \oint_{\mathcal{C}} A_{\mu}(x) d x^{\mu}} .
$$

Let us pointing out that the manifestly gauge invariant Wilson loop does not depend in (2.33) on the point $x_{1}$ from which is parametrized the loop $\mathcal{C}$ because of the colour trace $\operatorname{Tr}_{c}$. We have thus:

$$
G_{\text {stat. }}^{(4)}\left(x_{1}, x_{2}, x_{1}^{\prime}, x_{2}^{\prime}\right)=-S_{1}^{(0)}\left(x_{1}-x_{1}^{\prime}\right) S_{2}^{(0)}\left(x_{2}^{\prime}-x_{2}\right) W[\mathcal{C}]
$$

where $W[\mathcal{C}]$ is the gauge invariant one-loop functional:

$$
W[\mathcal{C}] \equiv \frac{1}{N_{c}}\langle\phi(\mathcal{C})\rangle_{A}=\frac{1}{Z} \int\left[d A_{\mu}(x)\right] \frac{1}{N_{c}} \phi(\mathcal{C}) e^{i S_{Y M}\left[A_{\mu}\right]} .
$$

Without loss of generality, let us consider the specific case where $x_{1}^{0}=x_{2}^{0} \equiv X^{0}$ and $x_{1}^{\prime 0}=x_{2}^{\prime 0} \equiv X^{\prime 0}$. The loop is then reduced to a rectangular contour (symbolized by $\square$ ) with temporal extent $T$ and spatial extent $r$. In the limit where $T \equiv\left(X^{0}-X^{\prime 0}\right) \rightarrow$ $\infty$ (in the static approximation, the distance between the fixed quark and antiquark $r \equiv\left|\vec{x}_{1}-\vec{x}_{2}\right|=\left|\vec{x}_{1}^{\prime}-\vec{x}_{2}^{\prime}\right|$ remains constant over time), the loop becomes infinitely stretched out and one finds Wilson's confinement criterion or area law [25]:

$$
W[\square] \underset{\square \rightarrow \infty}{=} e^{-i \sigma_{t} A[\square]}
$$

where $\sigma_{t}$ is the string tension and $A[\square]$ is the area of the minimal surface with the rectangle as boundary. In the relativistic flux tube model [26] in which the confinement results from the formation of a chromo-electric field tube (the effective "QCD string") between the quark and the antiquark, the constant $\sigma_{t}=\frac{1}{2 \pi \alpha^{\prime}}$, of the order of $0.18 \mathrm{GeV}^{2}$, stands for the linear energy density of the tube and is related to the slope $\alpha^{\prime}$ of the Regge trajectories: $J\left(m^{2}\right)=\alpha_{0}+\alpha^{\prime} m^{2}\left(J\right.$ and $m^{2}$ are the total spin and the square mass of the hadrons while $\alpha_{0}$ is the intercept). As a final result, we obtain:

$$
G_{\text {stat. }}^{(4)}\left(x_{1}, x_{2}, x_{1}^{\prime}, x_{2}^{\prime}\right)=-\delta^{3}\left(\vec{x}_{1}-\vec{x}_{1}^{\prime}\right) \delta^{3}\left(\vec{x}_{2}^{\prime}-\vec{x}_{2}\right) e^{-i m_{1} T} e^{-i m_{2} T} e^{-i \sigma_{t} r T} .
$$

On the other hand, it is possible to extract explicitly the energy dependence of the evolution amplitude of the quarkonium, as defined in (2.24). In the Heisenberg representation, the operators depend on the time and their dynamics are governed by the Hamiltonian of the system:

$$
\phi\left(\vec{x}_{1}, \vec{x}_{2}, X^{0}\right)=e^{i H X^{0}} \phi\left(\vec{x}_{1}, \vec{x}_{2}, 0\right) e^{-i H X^{0}} .
$$

This relation can also be understood as a particular case of the space-time translation:

$$
\phi(x)=e^{i p \cdot x} \phi(0) e^{-i p \cdot x}
$$


where $p=(H, \overrightarrow{0})$ is the energy-momentum operator. Thus we have:

$$
\begin{aligned}
G_{\text {stat. }}^{(4)}\left(x_{1}, x_{2}, x_{1}^{\prime}, x_{2}^{\prime}\right) & =\frac{1}{N_{c}}\left\langle 0\left|e^{i H X^{0}} \phi^{\dagger}\left(x_{1}, x_{2}\right) e^{-i H X^{0}} e^{i H X^{\prime 0}} \phi\left(x_{1}^{\prime}, x_{2}^{\prime}\right) e^{-i H X^{\prime 0}}\right| 0\right\rangle \\
& =\frac{1}{N_{c}}\left\langle 0\left|\phi^{\dagger}\left(\vec{x}_{1}, \vec{x}_{2}, 0\right) e^{-i H\left(X^{0}-X^{\prime 0}\right)} \phi\left(\vec{x}_{1}^{\prime}, \vec{x}_{2}^{\prime}, 0\right)\right| 0\right\rangle \\
& =\frac{1}{N_{c}}\left\langle\phi\left(\vec{x}_{1}, \vec{x}_{2}, 0\right)\left|e^{-i H T}\right| \phi\left(\vec{x}_{1}^{\prime}, \vec{x}_{2}^{\prime}, 0\right)\right\rangle .
\end{aligned}
$$

Let $\left\{\left|P_{n}\right\rangle\right\}$ be a complete set of eigenvectors of $H$ associated to the eigenvalues $\left\{E_{n}\right\}$ :

$$
\begin{aligned}
& H\left|P_{n}\right\rangle=E_{n}\left|P_{n}\right\rangle, \\
& \sum_{n}\left|P_{n}\right\rangle\left\langle P_{n}\right|=1 .
\end{aligned}
$$

If one puts the closure relation (2.43) into (2.40), one obtains:

$$
G_{\text {stat. }}^{(4)}\left(x_{1}, x_{2}, x_{1}^{\prime}, x_{2}^{\prime}\right)=\sum_{n} \frac{1}{N_{c}}\left\langle\phi\left(\vec{x}_{1}, \vec{x}_{2}, 0\right) \mid P_{n}\right\rangle\left\langle P_{n} \mid \phi\left(\vec{x}_{1}^{\prime}, \vec{x}_{2}^{\prime}, 0\right)\right\rangle e^{-i E_{n} T}
$$

In the limit $T \rightarrow \infty$, the leading contribution corresponds to the quarkonium ground-state $\left|P_{0}\right\rangle$ with the energy $E_{0}$ in the complex exponential function:

$$
G_{\text {stat. }}^{(4)}\left(x_{1}, x_{2}, x_{1}^{\prime}, x_{2}^{\prime}\right)=\frac{1}{N_{c}}\left\langle\phi\left(\vec{x}_{1}, \vec{x}_{2}, 0\right) \mid P_{0}\right\rangle\left\langle P_{0} \mid \phi\left(\vec{x}_{1}^{\prime}, \vec{x}_{2}^{\prime}, 0\right)\right\rangle e^{-i E_{0} T}
$$

where $\frac{1}{\sqrt{N_{c}}}\left\langle\phi\left(\vec{x}_{1}, \vec{x}_{2}, 0\right) \mid P_{0}\right\rangle$ is the wave function of the bound-state. One can understand the ground state of the quarkonium as the one in which the number of quark-gluon and gluon-gluon interactions is the smallest. In the static limit where the kinetic energies of the quark and antiquark vanish, we find:

$$
E_{0}=m_{1}+m_{2}+V(r)
$$

In comparison with (2.37), we infer the confining linear static potential $V(r)$ of an infinitely massive quark-antiquark pair:

$$
V(r)=-\lim _{T \rightarrow \infty} \frac{1}{i T} \ln W[\square]=\sigma_{t} r
$$

The only condition for the validity of the Feynman-Kac formula (2.47) is that the states $|\phi\rangle$ 's have a non-vanishing component over the ground state. 


\section{The heavy quark potential in the $A d S /$ CFT corre- spondence}

The gauge/string duality aims at relating gauge theory observables to calculations in higher-dimensional dual space-times. For instance, the method for deriving conformal dimensions of operators and correlators in conformal field theory via dual string theory has been described in $[2,3]$. On the other hand, J. M. Maldacena considered the problem of calculating the (expectation value of the) Wilson loop $W[\mathcal{C}][4]$. He suggested the following $A d S / \mathrm{CFT}$ duality relation:

$$
W[\mathcal{C}] \sim Z_{\text {string }}[\mathcal{C}]
$$

where $Z_{\text {string }}[\mathcal{C}]$ is the full partition function of the dual string theory. As previously discussed in the Introduction, although the string theory is weakly coupled in the 't Hooft limit ( $g_{s}$ and $g_{Y M}$ are the closed string and the Yang-Mills coupling constants respectively):

$$
g_{s}=\frac{g_{Y M}^{2}}{2 \pi}=\frac{1}{2 \pi} \frac{\lambda}{N} \underset{\substack{N \rightarrow \infty \\ \lambda \text { fixed }}}{\rightarrow \rightarrow} 0,
$$

we do not known yet how to solve free string theory on $A d S_{5} \times S^{5}$. Nevertheless, in the case where the 't Hooft coupling is large $\lambda \equiv g_{Y M}^{2} N \gg 1$, the typical length scale of the string $\ell_{s}=\sqrt{\alpha^{\prime}}$ is small in comparison with the $A d S_{5}$ radius $R$ :

$$
\frac{R^{4}}{\alpha^{\prime 2}}=4 \pi g_{s} N=2 \lambda \gg 1
$$

This is the so-called supergravity limit where strings appear as point-like particles and the stringy effects can be neglected. The $A d S / C F T$ prescription consists then in calculating the Wilson loop $W[\mathcal{C}]$ in terms of the proper area of a string world-sheet which describes the closed loop $\mathcal{C}$ on the boundary:

$$
W[\mathcal{C}] \sim e^{-S[\mathcal{C}]}
$$

where $S[\mathcal{C}]$ is the classical (Euclidean) action of the string world-sheet. As a matter of fact, the world-sheet does not describe the Wilson loop (2.35) but its supersymmetric generalization since $A d S_{5} \times S^{5}$ is the actual holographic space of the $\mathcal{N}=4 S U(N)$ super-Yang-Mills theory. Especially, we do not expect to necessarily recover the area law (2.36) of the Wilson loop (which is indeed a four-dimensional space-time result).

Since we consider an infinitely heavy $Q \bar{Q}$ pair (non-dynamical external probes), the inter-quark distance $r$ is a constant of time and the string connecting the quark to the 
antiquark gives rise to a rectangular contour $\mathcal{C}$ on the boundary with temporal extent $T$ and spatial extent $r$ :

$$
\begin{gathered}
-\frac{T}{2} \leq t \leq \frac{T}{2}, \\
-\frac{r}{2} \leq x \leq \frac{r}{2} .
\end{gathered}
$$

The quark $Q$ and the antiquark $\bar{Q}$ are put down at $x=r / 2$ and $x=-r / 2$ respectively. Nevertheless, because the string is now allowed to move along the fifth holographic coordinate of $A d S_{5}$, the minimal area of the world-sheet, of which the boundary is the loop $\mathcal{C}$, is no longer the rectangle. Indeed, $A d S_{5}$ is a curved space-time (the gravity effects are non-zero) and, as a result, the string world-sheet will not span only the four-dimensional surface enclosed by the contour $\mathcal{C}$ at the boundary $\partial A d S_{5}$. Instead of that, the string will move inside the bulk and, held back by its tension, will reach an extremal value of the holographic coordinate.

If we choose the Poincaré coordinates $x^{M}=\left(x^{\mu}, z\right)(\mu=0,1,2,3)$, the $A d S_{5} \times S^{5}$ line element reads (throughout this section, we will work with the Euclidean signature):

$$
d s^{2}=\frac{R^{2}}{z^{2}}\left(\delta_{\mu \nu} d x^{\mu} d x^{\nu}+d z^{2}\right)+R^{2} d \Omega_{5}^{2} .
$$

$A d S_{5}$ is the domain $z>0$ and $\delta_{\mu \nu}=\operatorname{diag}(+1,+1,+1,+1)$ is the Euclidean metric tensor of the boundary space-time $\partial A d S_{5}$ which can be defined by multiplying (3.54) by $z^{2}$ and setting $z=0$. Let us then define the dimensionless $A d S$ radius $\tilde{R}$ as:

$$
\frac{R^{4}}{\alpha^{\prime 2}} \equiv \tilde{R}^{4}
$$

We also introduce a new holographic coordinate:

$$
u=\frac{R^{2}}{\alpha^{\prime} z}=\frac{\tilde{R}^{2}}{z}
$$

which has the dimension of an energy. Not surprisingly, the line element of the bulk (3.54) becomes (1.6):

$$
d s^{2}=g_{M N}(x) d x^{M} d x^{N}+R^{2} d \Omega_{5}^{2}=\alpha^{\prime}\left(\frac{u^{2}}{\tilde{R}^{2}} \delta_{\mu \nu} d x^{\mu} d x^{\nu}+\frac{\tilde{R}^{2}}{u^{2}} d u^{2}\right)+R^{2} d \Omega_{5}^{2} .
$$

To the high (low) energy region $z \rightarrow 0(z \rightarrow+\infty)$ corresponds $u \rightarrow+\infty(u \rightarrow 0)$.

Let us consider the simplest action which describes the dynamics of an open string, namely the so-called Nambu-Goto action:

$$
S_{N G}[\mathcal{C}]=T_{0} \int d^{2} \xi \sqrt{\operatorname{det}\left(\gamma_{a b}\right)}
$$


$T_{0}^{-1}=2 \pi \alpha^{\prime}$ is the (inverse of the) fundamental string tension and $\gamma_{a b}(\xi)(a, b=1,2)$ is the induced metric tensor on the two-dimensional world-sheet:

$$
\gamma_{a b}(\xi) \equiv G_{M N}(X) \frac{\partial X^{M}}{\partial \xi^{a}} \frac{\partial X^{N}}{\partial \xi^{b}}
$$

which requires two parameters $\xi^{1} \equiv \sigma$ and $\xi^{2} \equiv \tau$ (the measure is $d^{2} \xi=d \sigma d \tau$ ). The metric tensor $G_{M N}(X)$ of the bulk (3.57) in written in terms of the world-sheet coordinates $X^{M}\left(\xi^{a}\right)=\left(X^{0}, X^{1}, X^{2}, X^{3}, X^{5} \equiv U\right)\left(\xi^{a}\right)(M=\mu, 5$ and, following a standard convention in string theory, these coordinates (and the metric) are denoted with capital letters). In static configuration, an useful parametrization of the string world-sheet is the so-called static gauge where:

$$
X^{0}(\tau, \sigma) \equiv t(\tau, \sigma)=\tau
$$

The lines of constant $\tau$ are "static strings" in the chosen Lorentz frame. On the other hand, according to the reparametrization invariance of the Nambu-Goto action (3.58), we can choose:

$$
\sigma=x
$$

According to (2.47), the energy of a quark-antiquark pair is obtained in the limit $T \rightarrow \infty$. The string world-sheet is then invariant under a translation along the time coordinate and symmetric under the mirror transformation $x \leftrightarrow-x$. Consequently, the holographic coordinate of the string $U(x)$, a function of $x$ only, presents a minimum which, by symmetry, occurs at $x=0$ : in the sequel, we will denote $U_{0} \equiv U(0)$ this noteworthy value. Let us express explicitly the Nambu-Goto action (3.58) in terms of the string coordinates. For this, we have to evaluate the components of the induced metric tensor $(3.59)$ :

$$
\begin{aligned}
\gamma_{11} & =G_{11}(X) \frac{\partial X^{1}}{\partial \sigma} \frac{\partial X^{1}}{\partial \sigma}+G_{55}(X) \frac{\partial U}{\partial \sigma} \frac{\partial U}{\partial \sigma}=\alpha^{\prime} \frac{U^{2}}{\tilde{R}^{2}}+\alpha^{\prime} \frac{\tilde{R}^{2}}{U^{2}} U^{\prime 2} \\
\gamma_{12} & =\gamma_{21}=G_{M N}(X) \frac{\partial X^{M}}{\partial \sigma} \frac{\partial X^{N}}{\partial \tau}=0, \\
\gamma_{22} & =G_{00}(X) \frac{\partial X^{0}}{\partial \tau} \frac{\partial X^{0}}{\partial \tau}=\alpha^{\prime} \frac{U^{2}}{\tilde{R}^{2}}
\end{aligned}
$$

such that

$$
S_{N G}[\mathcal{C}]=T_{0} \int_{-T / 2}^{T / 2} d t \int_{-r / 2}^{r / 2} d x \mathcal{L}\left(U(x), U^{\prime}(x)\right)
$$

with the Lagrangian density

$$
\mathcal{L}=\alpha^{\prime} \sqrt{U^{\prime 2}+\frac{U^{4}}{\tilde{R}^{4}}}
$$


and where the prime denotes the derivative with respect to the spatial coordinate $d U=$ $U^{\prime}(x) d x$. The shape $U(x)$ of the string world-sheet stretching into the bulk is governed by the Euler-Lagrange equation:

$$
\delta_{U} S_{N G}=0 \Rightarrow \frac{\partial \mathcal{L}}{\partial U}-\frac{d}{d x} \frac{\partial \mathcal{L}}{\partial U^{\prime}}=0 \Rightarrow U U^{\prime \prime}-4 U^{\prime 2}-2 \frac{U^{4}}{\tilde{R}^{4}}=0 .
$$

Moreover, since the Lagrangian density (3.66) does not depend explicitly on $x$, we have the following first integral:

$$
\frac{d \mathcal{L}}{d x}=U^{\prime} \frac{\partial \mathcal{L}}{\partial U}+U^{\prime \prime} \frac{\partial \mathcal{L}}{\partial U^{\prime}}=\frac{d}{d x}\left(U^{\prime} \frac{\partial \mathcal{L}}{\partial U^{\prime}}\right)+U^{\prime}\left(\frac{\partial \mathcal{L}}{\partial U}-\frac{d}{d x} \frac{\partial \mathcal{L}}{\partial U^{\prime}}\right)=0
$$

The last term vanishes according to the equation of motion (3.67) and it remains:

$$
\frac{d}{d x}\left(U^{\prime} \frac{\partial \mathcal{L}}{\partial U^{\prime}}-\mathcal{L}\right)=0
$$

which finally gives:

$$
\frac{U^{4}}{\sqrt{U^{\prime 2}+\frac{U^{4}}{\tilde{R}^{4}}}}=\tilde{R}^{2} U_{0}^{2}=\text { const. }
$$

The constant can be evaluated, for instance, at $x=0$ where $U(0)=U_{0}$ and $U^{\prime}(0)=0$ by symmetry. One can also derived a useful relation for the derivative of the string coordinate (the positive (negative) sign corresponds to $0<x<r / 2(-r / 2<x<0)$ ):

$$
U^{\prime}(x)= \pm \frac{U^{2}}{\tilde{R}^{2} U_{0}^{2}} \sqrt{U^{4}-U_{0}^{4}}
$$

At this stage, it is suitable to determine the dependence of the quark-antiquark distance $r$ on the holographic string coordinate. To do so, we start from the following integral:

$$
x=\int_{0}^{x} d x^{\prime}=\int_{U_{0}}^{U(x)} \frac{d U}{U^{\prime}\left(x^{\prime}\right)}=\int_{U_{0}}^{U(x)} \frac{d U}{U^{2}} \frac{\tilde{R}^{2} U_{0}^{2}}{\sqrt{U^{4}-U_{0}^{4}}} \underset{\left(v \equiv \frac{U}{U_{0}}\right)}{=} \frac{\tilde{R}^{2}}{U_{0}} \int_{1}^{U(x) / U_{0}} \frac{d v}{v^{2} \sqrt{v^{4}-1}} .
$$

For $x=\frac{r}{2}$ (where one puts the heavy quark $Q$ ), $U\left(\frac{r}{2}\right) \rightarrow+\infty$ such that

$$
r\left(U_{0}\right)=\frac{2 \tilde{R}^{2}}{U_{0}} \int_{1}^{\infty} \frac{d v}{v^{2} \sqrt{v^{4}-1}}=\frac{\tilde{R}^{2}}{U_{0}} \frac{1}{\rho}
$$

where we have defined the numerical factor:

$$
\rho=\frac{\Gamma(1 / 4)^{2}}{(2 \pi)^{3 / 2}}
$$

This relation could have also been guessed from the underlying conformal nature of the theory since $r \rightarrow \lambda r$ under a dilatation $z \rightarrow \lambda z$, i.e. when $U_{0} \rightarrow \frac{U_{0}}{\lambda}$ (see Eq.(3.56)). For later convenience, it is also straightforward to express $U_{0}$ in terms of $r$ :

$$
U_{0}(r)=\frac{\tilde{R}^{2}}{\rho} \frac{1}{r} .
$$


We are now ready to derive, at least naively, the static $V_{Q \bar{Q}}(r)$ potential. The Euclidean version of the Feynman-Kac formula (2.47) allows us to write:

$$
V_{Q \bar{Q}}(r)=\lim _{T \rightarrow \infty} \frac{1}{T} S_{N G}[\mathcal{C}]=\frac{1}{2 \pi} \int_{-r / 2}^{r / 2} d x \sqrt{U^{\prime 2}+\frac{U^{4}}{\tilde{R}^{4}}}
$$

Using successively the properties of the holographic coordinate $(U(-x)=U(x)$ and $\left.U^{\prime}(-x)=-U^{\prime}(x)\right)$, the first integral (3.70) and the expression of the derivative $U^{\prime}(x)$ (3.71), we have:

$$
\begin{aligned}
& V_{Q \bar{Q}}(r)=\frac{1}{\pi} \int_{0}^{r / 2} d x \sqrt{U^{\prime 2}+\frac{U^{4}}{\tilde{R}^{4}}}=\frac{1}{\pi} \int_{U_{0}}^{\infty} \frac{d U}{U^{\prime}} \frac{U^{4}}{\tilde{R}^{2} U_{0}^{2}} \\
& =\frac{1}{\pi \tilde{R}^{2} U_{0}^{2}} \int_{U_{0}}^{\infty} d U U^{4} \frac{\tilde{R}^{2} U_{0}^{2}}{U^{2} \sqrt{U^{4}-U_{0}^{4}}} \underset{\left(v \equiv \frac{U}{U_{0}}\right)}{=} \frac{U_{0}}{\pi} \int_{1}^{\infty} d v \frac{v^{2}}{\sqrt{v^{4}-1}} .
\end{aligned}
$$

As a matter of fact, the last integral gives rise to an infinite result: the potential indeed needs to be regularized according to the prescription $U(x) \leq U_{\max }$ (the limit $U_{\max } \rightarrow+\infty$ being taken at the end of the calculations). Actually, the final recipe for computing the Wilson loop, as proposed by Maldacena [4], turns out to be:

$$
V_{Q \bar{Q}}^{(R)}(r)=\lim _{\substack{T \rightarrow \infty \\ M \rightarrow \infty}} \frac{1}{T}\left(S_{N G}-\ell M\right)
$$

where $\ell$ is the perimeter of the loop $\mathcal{C}$ on the boundary:

$$
\ell=2 T+2 r \underset{T \gg r}{=} 2 T
$$

which amounts, in the limit $T \rightarrow \infty$, to twice the temporal extent $T$ of the contour. $M$ is the mass of the so-called "W-boson string" associated to the quarks [4]. For infinitely massive quark and antiquark, this open string stretches all the way from $U=0$ (one brane which is far away from the boundary) to $U=\infty$ (where $N$ coincident D3-branes defines, at low energies, a $S U(N)$ Yang-Mills theory). In a flat space-time, the mass-squared $M^{2}$ of a stretched string reads as (the quantum fluctuations are neglected) ${ }^{2}$ :

$$
M^{2}=\left(\frac{x_{2}^{a}-x_{1}^{a}}{2 \pi \alpha^{\prime}}\right)^{2}
$$

\footnotetext{
${ }^{2}$ In a $d$-dimensional flat space-time, an open string between two parallel $\mathrm{D} p$ - and $\mathrm{D} q$-branes, located respectively at $x_{1}$ and $x_{2}$, has the following square mass spectrum $(p>q)$ :

$$
M^{2}=\left(\frac{x_{2}^{a}-x_{1}^{a}}{2 \pi \alpha^{\prime}}\right)^{2}+\frac{1}{\alpha^{\prime}}\left(N^{(i, r, a)}-1+\frac{1}{16}(p-q)\right)
$$

with the number operator $N^{(i, r, a)}=\sum_{n=1}^{\infty} \sum_{i=2}^{q} n a_{n}^{i \dagger} a_{n}^{i}+\sum_{k \in \mathbb{Z}_{\text {odd }}^{+}} \sum_{r=q+1}^{p} \frac{k}{2} a_{\frac{k}{2}}^{r \dagger} a_{\frac{k}{2}}^{r}+\sum_{m=1}^{\infty} \sum_{a=p+1}^{d} m a_{m}^{a \dagger} a_{m}^{a}$. The indices $i$ and $a$ refer respectively to the tangential and to the normal directions to the two branes while the index $r$ stands for the remaining tangential coordinates for the $\mathrm{D} p$-brane and, thus, additional normal coordinates to the $\mathrm{D} q$-brane.
} 
The locations of the two parallel, separated D-branes, on which lie each of the endpoints of the open string, are specified by the values $x_{1}^{a}$ and $x_{2}^{a}$ of the coordinates normal, respectively, to the first and to the second branes. In our case, the bulk is $A d S_{5}$ and the superscript $a$ corresponds to the only normal coordinate to the branes, i.e. the fifth holographic coordinate $(a=5)$. Since the string tension is $T_{0}=\frac{1}{2 \pi \alpha^{\prime}}$, one sees that (3.81) is simply the square of the energy of a classical static string stretched between two Dbranes. In short, the second term on the r.h.s. of (3.78) consists in subtracting in the Nambu-Goto action $S_{N G}$ the contribution of the (infinitely stretched) string associated to the (infinitely massive) quark and antiquark. In (3.81), the ratio $\frac{x}{\alpha^{\prime}}$ has the dimension of an energy and, therefore, can be identified to the holographic coordinate $U(x)$. The regularized mass of the "W-boson string" (in string theory, such a stretched open string corresponds to a massive vector field) is

$$
M=\frac{U_{\max }}{2 \pi}
$$

such that

$$
\begin{aligned}
V_{Q \bar{Q}}^{(R)}(r) & =\frac{1}{2 \pi} \int_{-r / 2}^{r / 2} d x \sqrt{U^{\prime 2}+\frac{U^{4}}{\tilde{R}^{4}}}-\frac{U_{\max }}{\pi} \\
& =\frac{U_{0}}{\pi} \int_{1}^{U_{\max } / U_{0}} d v \frac{v^{2}}{\sqrt{v^{4}-1}}-\frac{U_{\max }}{\pi} \\
& =\frac{U_{0}}{\pi} \int_{1}^{U_{\max } / U_{0}} d v\left(\frac{v^{2}}{\sqrt{v^{4}-1}}-1\right)+\frac{U_{0}}{\pi} \int_{1}^{U_{\max } / U_{0}} d v-\frac{U_{\max }}{\pi} \\
V_{Q \bar{Q}}^{(R)}(r) & =\frac{U_{0}}{\pi}\left[\int_{1}^{U_{\max } / U_{0}} d v\left(\frac{v^{2}}{\sqrt{v^{4}-1}}-1\right)-1\right] .
\end{aligned}
$$

The integral turns out to be finite in the limit $U_{\max } \rightarrow+\infty$ with the result:

$$
\begin{aligned}
V_{Q \bar{Q}}^{(R)}(r) & =-\frac{U_{0}}{\pi}(E(-1)-(2-i) K(-1)+K(2)) \\
& =-\frac{U_{0}}{\pi}(E(-1)-K(-1)),
\end{aligned}
$$

expressed in terms of the complete Elliptic Integrals $K(z)$ and $E(z)$ of the first and second kind respectively and where we have used the relation

$$
K(1 / z)=\sqrt{z}\left(K(z)-\sqrt{-\frac{1}{z}} \sqrt{\frac{1}{1-z}} \sqrt{z(1-z)} K(1-z)\right)
$$

with $z=-1$. According to (3.75) and to the numerical values of $K(-1)$ and $E(-1)$ :

$$
\begin{aligned}
K(-1) & =\frac{\Gamma(1 / 4)^{2}}{4 \sqrt{2 \pi}}=\frac{\pi}{2} \rho \\
E(-1) & =\frac{2 \Gamma(3 / 4)^{4}+\pi^{2}}{2 \sqrt{2 \pi} \Gamma(3 / 4)^{2}}=\frac{1}{2 \rho}+\frac{\pi}{2} \rho,
\end{aligned}
$$


it is possible to rewrite the static potential as:

$$
V_{Q \bar{Q}}^{(R)}(r)=-\frac{\tilde{R}^{2}}{2 \pi \rho^{2}} \frac{1}{r}
$$

or, in terms of the 't Hooft coupling $\lambda(3.50)$ :

$$
V_{Q \bar{Q}}^{(R)}(r)=-\frac{4 \pi^{2} \sqrt{2 \lambda}}{\Gamma(1 / 4)^{4}} \frac{1}{r}
$$

This result is valid for all distances $r$ when $\lambda=g_{Y M}^{2} N$ is large independently of the value of $g_{Y M}$. Especially, we do not recover the area law. Moreover, although the potential seems to have a short-distance Coulomb-like behaviour in $1 / r$ (a fact which is determined by conformal invariance: the factor $1 / \alpha^{\prime}$ in the string tension disappears in (3.65) and, consequently, in the expression of the potential (3.83)), it goes as $\sqrt{\lambda}$ instead of $\lambda$ which is the perturbative one-loop result: actually, the potential (3.89) turns out to be intrinsically non-perturbative.

\section{The static potential at finite temperature}

\subsection{The conformal behaviour of the Wilson loop at finite temper- ature}

Following Hawking and Page's work on the thermodynamics of black holes in the antide Sitter space-time $A d S_{4}$ [27], a gauge/string duality involving a gauge theory at finite temperature was proposed by Witten [8]. In this framework, on the supergravity side, the $A d S_{5}$ space-time (3.57) turns out to accommodate a Schwarzschild black hole (BH) with (the Euclidean version of) the metric:

$$
d s_{B H}^{2}=\alpha^{\prime}\left\{\frac{u^{2}}{\tilde{R}^{2}}\left(f(u) d t^{2}+\sum_{i=1}^{3} d x_{i}^{2}\right)+\frac{\tilde{R}^{2}}{u^{2}} \frac{d u^{2}}{f(u)}+\tilde{R}^{2} d \Omega_{5}^{2}\right\} .
$$

We have defined:

$$
f(u)=1-\frac{u_{T}^{4}}{u^{4}}
$$

and $\tilde{R}^{2}$ as in (3.55). $d \Omega_{5}^{2}$ is the line element of the unit radius compact 5 -sphere $S^{5}$. There is a curvature singularity in the IR at $u=0$ hidden behind an event horizon at $u=u_{T}$. In particular, at zero temperature, which corresponds to $u_{T}=0$ as we shall see below (4.95), we recover the metric of the $A d S_{5} \times S^{5}$ space-time (3.57). Such a solution (with an event horizon) is also called the near-extremal D3-brane solution of the equations of motion for the metric in type IIB superstring theory, the extremal case corresponding to the absence of horizon $u_{T}=0$ and $f(u)=1$, i.e. to the zero temperature case. 
The Euclidean time direction shrinks to a zero-size geometrical point at the horizon since $f\left(u_{T}\right)=0$ and is thus compactified on a circle with period $\beta: t \sim t+\beta$. This period $\beta=\frac{1}{T}$ is the inverse of the Beckenstein-Hawking temperature (which corresponds to the thermal temperature of the gauge theory) of the near-extremal solution (4.90) and gives the location $u_{T}$ of the horizon. We can reason as in [28]. The region of interest here is near $u_{T}$. So, let us define a new holographic coordinate $\rho$ as $u(\rho)=u_{T}\left(1+\frac{\rho^{2}}{\alpha^{\prime} \tilde{R}^{2}}\right)$. The relevant two-dimensional part of the metric (4.90) becomes:

$$
\begin{aligned}
d s_{B H}^{2}= & \alpha^{\prime} \frac{u^{2}}{\tilde{R}^{2}} f(u) d t^{2}+\frac{\tilde{R}^{2}}{u^{2}} \frac{d u^{2}}{f(u)}+\ldots \\
= & \frac{\alpha^{\prime}}{\tilde{R}^{2}} u_{T}^{2}\left(1+\frac{\rho^{2}}{\alpha^{\prime} \tilde{R}^{2}}\right)^{2}\left[1-\left(1+\frac{\rho^{2}}{\alpha^{\prime} \tilde{R}^{2}}\right)^{-4}\right] d t^{2} \\
& +\frac{\alpha^{\prime} \tilde{R}^{2}}{u_{T}^{2}}\left(1+\frac{\rho^{2}}{\alpha^{\prime} \tilde{R}^{2}}\right)^{-2}\left[1-\left(1+\frac{\rho^{2}}{\alpha^{\prime} \tilde{R}^{2}}\right)^{-4}\right]^{-1} \frac{4 u_{T}^{2}}{\alpha^{\prime 2} \tilde{R}^{4}} \rho^{2} d \rho^{2}+\ldots \\
d s_{B H}^{2}= & d \rho^{2}+\rho^{2} d\left(\frac{2 u_{T}}{\tilde{R}^{2}} t\right)^{2}+\ldots
\end{aligned}
$$

We then recognize on the r.h.s. of (4.92) the (dimensionless) angle $\theta \equiv \frac{2 u_{T}}{\widetilde{R}^{2}} t$ with period $2 \pi$ such that $\frac{2 u_{T}}{\tilde{R}^{2}} \beta=2 \pi$ or $u_{T}=\pi \tilde{R}^{2} T$. The Beckenstein-Hawking temperature can also be derived from the formula ( $\delta_{00}$ is the Euclidean metric component with time indices):

$$
\begin{aligned}
T & =\left.\frac{1}{4 \pi \alpha^{\prime}} \frac{\partial \delta_{00}}{\partial u}\right|_{u=u_{T}} \\
& =\left.\frac{1}{4 \pi \alpha^{\prime}} \frac{\partial}{\partial u}\left(\alpha^{\prime} \frac{u^{2}}{\tilde{R}^{2}} f(u)\right)\right|_{u=u_{T}} \\
T & =\frac{u_{T}}{\pi \tilde{R}^{2}} .
\end{aligned}
$$

We still work with a space-time Wilson loop (or ordinary Wilson loop, in contrast to the spatial Wilson loop that we shall consider in the non-conformal cases in the next sections) for which the boundary temporal and spatial extents are given by (3.52) and (3.53). In the static gauge $X^{0}(\tau, \sigma) \equiv t=\tau$ (3.60) and $\sigma=x$ (3.61), the Nambu-Goto action is [29]:

$$
S_{N G}[\mathcal{C}]=\frac{1}{2 \pi \alpha^{\prime}} \int d^{2} \xi \sqrt{\operatorname{det}\left(\gamma_{a b}\right)}=\frac{T}{2 \pi} \int_{-r / 2}^{r / 2} d x \sqrt{U^{\prime 2}+\frac{U^{4}-U_{T}^{4}}{\tilde{R}^{4}}}
$$

with

$$
\begin{aligned}
\gamma_{11} & =\alpha^{\prime} \frac{U^{2}}{\tilde{R}^{2}}+\alpha^{\prime} \frac{\tilde{R}^{2}}{U^{2}} \frac{U^{\prime 2}}{f(U)}, \\
\gamma_{22} & =\alpha^{\prime} \frac{U^{2}}{\tilde{R}^{2}} f(U)
\end{aligned}
$$


the non-vanishing components of the induced metric tensor on the two-dimensional worldsheet (with our conventions, $\xi^{1}=\sigma$ and $\xi^{2}=\tau$ ). $U^{\prime} \equiv U^{\prime}(x)$ is the derivative of the holographic coordinate with respect to the spatial boundary coordinate $x$. The Lagrangian density does not depend explicitly on this $x$. Thus, the Hamiltonian in the $x$ direction is a constant of motion and the first integral (3.69) can be evaluated at $x=0$ where $U(0)=U_{0}$ and $U^{\prime}(0)=0$ by symmetry. We obtain:

$$
\frac{U^{4}-U_{T}^{4}}{\sqrt{U^{\prime 2}+\frac{U^{4}-U_{T}^{4}}{\tilde{R}^{4}}}}=\tilde{R}^{2} \sqrt{U_{0}^{4}-U_{T}^{4}}=\text { const. }
$$

from which can be derived an expression for the derivative $U^{\prime}(x)$ :

$$
U^{\prime}(x)= \pm \frac{\sqrt{\left(U^{4}-U_{T}^{4}\right)\left(U^{4}-U_{0}^{4}\right)}}{\tilde{R}^{2} \sqrt{U_{0}^{4}-U_{T}^{4}}}
$$

where the positive (negative) square root corresponds to $0<x \leq \frac{r}{2}\left(-\frac{r}{2} \leq x<0\right)$.

The integral expression for the distance $r$ between the quark and the antiquark is derived as usual $(x \geq 0)$ :

$$
\frac{r}{2}-x=\int_{x}^{r / 2} d x^{\prime}=\int_{U(x)}^{\infty} \frac{d U}{U^{\prime}}=\tilde{R}^{2} \sqrt{U_{0}^{4}-U_{T}^{4}} \int_{U(x)}^{\infty} \frac{d U}{\sqrt{\left(U^{4}-U_{T}^{4}\right)\left(U^{4}-U_{0}^{4}\right)}}
$$

With respect to $v \equiv U / U_{0}$ and defining $\epsilon \equiv f\left(U_{0}\right)=1-\frac{U_{T}^{4}}{U_{0}^{4}} \geq 0$ (since $U_{0} \geq U_{T}$ ), we get:

$$
\frac{r}{2}-x=\frac{\tilde{R}^{2}}{U_{0}} \sqrt{\epsilon} \int_{U(x) / U_{0}}^{\infty} \frac{d v}{\sqrt{\left(v^{4}-1+\epsilon\right)\left(v^{4}-1\right)}}
$$

In particular, if $x=0$, then

$$
r\left(U_{0}, U_{T}\right)=\frac{2 \tilde{R}^{2}}{U_{0}} \sqrt{\epsilon} \int_{1}^{\infty} \frac{d v}{\sqrt{\left(v^{4}-1+\epsilon\right)\left(v^{4}-1\right)}} .
$$

In the supergravity approach, the static potential stemming from the space-time Wilson loop with the background (4.90) is obtained as follows. Thanks to the first integral (4.99), we have derived an expression for $U^{\prime}(4.100)$. It is then easy to rewrite the action (4.96) following the same steps as in (3.77):

$$
\begin{aligned}
S_{N G}[\mathcal{C}] & =\frac{T}{\pi} \int_{0}^{r / 2} d x \sqrt{U^{2}+\frac{U^{4}-U_{T}^{4}}{\tilde{R}^{2}}}=\frac{T}{\pi} \int_{0}^{r / 2} d x \frac{U^{4}-U_{T}^{4}}{\tilde{R}^{2} \sqrt{U_{0}^{4}-U_{T}^{4}}} \\
& =\frac{T}{\pi} \int_{U_{0}}^{\infty} \frac{d U}{U^{\prime}} \frac{U^{4}-U_{T}^{4}}{\tilde{R}^{2} \sqrt{U_{0}^{4}-U_{T}^{4}}} \underset{\left(v \equiv \frac{U}{U_{0}}\right)}{=} T \frac{U_{0}}{\pi} \int_{1}^{\infty} d v \sqrt{\frac{v^{4}-1+\epsilon}{v^{4}-1}} .
\end{aligned}
$$


The heavy quark potential is given by Maldacena's prescription (3.78):

$$
V_{Q \bar{Q}}^{(R)}(r)=\lim _{\substack{T \rightarrow \infty \\ M \rightarrow \infty}} \frac{1}{T}\left(S_{N G}-\ell M\right)
$$

where the regularization procedure introduces in the action $S_{N G}$ an ultraviolet (UV) cutoff $U \leq U_{\max }\left(v \leq U_{\max } / U_{0}\right)$. The second contribution on the r.h.s. of (4.105) is the required counter-term which subtracts the very massive quark and antiquark contributions to the regularized potential and which takes in this case the following form $(\ell=2 r+2 T \underset{T \gg r}{\widetilde{\widetilde{P}}} 2 T$ and $\left.M=\frac{U_{\max }-U_{T}}{2 \pi}\right)$ :

$$
V_{c . t .}=-\lim _{U_{\max } \rightarrow \infty} \frac{U_{\max }-U_{T}}{\pi} .
$$

Indeed, the "W-boson string" corresponds here to an open string stretched between the brane at $U_{\max }$ and the Schwarzschild horizon $U=U_{T}[29,30]$. Hence, the counter-term (4.106) and the renormalized (or, more properly, subtracted) static potential:

$$
\begin{aligned}
V_{Q \bar{Q}}^{(R)}\left(U_{0}, U_{T}\right) & =\frac{U_{0}}{\pi} \int_{1}^{U_{\max } / U_{0}} d v\left(\sqrt{\frac{v^{4}-1+\epsilon}{v^{4}-1}}-1\right)+\frac{U_{0}}{\pi} \int_{1}^{U_{\max } / U_{0}} d v-\frac{U_{\max }-U_{T}}{\pi} \\
& =\frac{U_{0}}{\pi} \int_{1}^{U_{\max } / U_{0}} d v\left(\sqrt{\frac{v^{4}-1+\epsilon}{v^{4}-1}}-1\right)+\frac{U_{T}-U_{0}}{\pi} .
\end{aligned}
$$

Let us focus on the limit case $U_{0} \gg U_{T}$ where the string world-sheet is close to the boundary such that it does not feel the presence of the horizon. In fact, this configuration corresponds to the low temperature limit $r \ll \beta$ or $r T \ll 1$ (we have $u_{T}=\pi \tilde{R}^{2} T$ ). Obviously, for small temperatures, the potential behaves approximately as in the zero temperature case (3.89) $V \sim-\frac{1}{r}$ since we recover for $\epsilon \simeq 1$ the expressions (3.73) and (3.83). Moreover, the leading non-zero temperature correction exhibits scaling consistent with the conformal invariance of the boundary theory. [29] obtained:

$$
V \propto-\frac{1}{r}\left(1+a(r T)^{4}\right)
$$

with $a$ a positive numerical constant which does not depend on $\tilde{R}$. Without length scale, it is indeed meaningless to speak, at low temperature, of a large or small compactification radius of the Euclidean temporal dimension.

The high temperature limit $r \gg \beta$ or $r T \gg 1$ when $U_{0} \simeq U_{T}$ is more subtle. As shown in $[29,30]$, there is a critical value of the inter-quark distance $r_{c}$ above which the potential starts to be positive. At this point, the bound-state equations (4.103) and (4.107) are no longer valid because the lowest energy configuration consists instead of two straight strings ending at the horizon. In other words, the quarks become free as screened by the effects of the temperature. Hence, the potential exhibits a behaviour expected for the deconfinement phase at high temperature when the meson decays into a configuration of quarks without interaction. 


\subsection{The area law in three-dimensional Yang-Mills theory}

Following $[8,31,32]$, we consider a spatial Wilson loop $W[\mathcal{C}]$ (along two space-like dimensions) at fixed value of the temperature and take the spatial extent $Y$ to be large with respect to the other spatial direction $Y \gg r$. We choose the following parametrization for the string world-sheet:

$$
\begin{gathered}
-\frac{r}{2} \leq x \leq \frac{r}{2}, \\
-\frac{Y}{2} \leq y \leq \frac{Y}{2} .
\end{gathered}
$$

In the limit $Y \rightarrow \infty$, the world-sheet configuration is invariant under translation in the $Y$ direction. It is then straightforward to write out the classical action (3.58) of the space-like Nambu-Goto string in the background (4.90). The relevant components of the induced metric tensor are this time $\left(\xi^{1}=x\right.$ and $\left.\xi^{2}=y\right)$ :

$$
\begin{aligned}
\gamma_{11} & =\alpha^{\prime} \frac{U^{2}}{\tilde{R}^{2}}+\alpha^{\prime} \frac{\tilde{R}^{2}}{U^{2}} \frac{U^{\prime 2}}{f(U)}, \\
\gamma_{22} & =\alpha^{\prime} \frac{U^{2}}{\tilde{R}^{2}}
\end{aligned}
$$

such that

$$
S_{N G}[\mathcal{C}]=\frac{1}{2 \pi \alpha^{\prime}} \int_{-Y / 2}^{Y / 2} d y \int_{-r / 2}^{r / 2} d x \sqrt{\operatorname{det}\left(\gamma_{a b}\right)}=\frac{Y}{2 \pi} \int_{-r / 2}^{r / 2} d x \sqrt{\frac{U^{4}}{\tilde{R}^{4}}+\frac{U^{4}}{U^{4}-U_{T}^{4}} U^{\prime 2}} .
$$

As always, since the Lagrangian density does not depend explicitly on $x$, we find a first integral which here takes the following form $\left(U(0) \equiv U_{0}\right.$ and $U^{\prime}(0)=0$ by symmetry):

$$
\frac{U^{4}}{\sqrt{\frac{U^{4}}{\tilde{R}^{4}}+\frac{U^{4}}{U^{4}-U_{T}^{4}} U^{\prime 2}}}=\tilde{R}^{2} U_{0}^{2}=\text { const. }
$$

or, in terms of the derivative $U^{\prime}(x)$ of the holographic coordinate:

$$
U^{\prime}(x)= \pm \frac{U^{2}}{\tilde{R}^{2}} \sqrt{\left(1-\frac{U_{T}^{4}}{U^{4}}\right)\left(\frac{U^{4}}{U_{0}^{4}}-1\right)} .
$$

The next step consists in deriving the expressions of the inter-quark distance and of the heavy quark potential as functions of $U_{0}$ and $U_{T}$. As for the distance $r\left(U_{0}, U_{T}\right)$, we have:

$$
\begin{aligned}
r\left(U_{0}, U_{T}\right) & =\int_{-r / 2}^{r / 2} d x=2 \int_{0}^{r / 2} d x=2 \int_{U_{0}}^{\infty} \frac{d U}{U^{\prime}}=2 \tilde{R}^{2} \int_{U_{0}}^{\infty} \frac{d U}{U^{2}} \frac{1}{\sqrt{\left(1-\frac{U_{T}^{4}}{U^{4}}\right)\left(\frac{U^{4}}{U_{0}^{4}}-1\right)}} \\
& =\frac{d v}{\left(v \equiv \tilde{R}^{2}\right.} \\
\frac{U_{0}}{U_{0}} & \int_{1}^{\infty} \frac{d .116}{\sqrt{\left(v^{4}-1+\epsilon\right)\left(v^{4}-1\right)}}
\end{aligned}
$$


with $\epsilon \equiv f\left(U_{0}\right)=1-\frac{U_{T}^{4}}{U_{0}^{4}}$. In the limit $U_{0} \simeq U_{T}(\epsilon \ll 1)$ where the string world-sheet reaches the horizon, the inter-quark distance diverges:

$$
r\left(U_{0}\right)=\frac{2 \tilde{R}^{2}}{U_{0}} \int_{a}^{\infty} d v \frac{1}{v^{4}-1} \underset{a \rightarrow 1}{\sim}-\frac{\tilde{R}^{2}}{2 U_{0}} \ln (a-1) .
$$

Thus, we see that the large distance limit (where the confinement is expected to appear) consists then in taking the limit $U_{0} \simeq U_{T}$. On the other hand, when $r \gg \beta=\frac{1}{T}$, the circle $S^{1}(\beta)$ around the compactified Euclidean time direction is small and, as a result, the number of dimensions of the gauge theory on the boundary reduces to three. By choosing appropriate boundary conditions along this circle (namely, by taking antiperiodic fermions around $S^{1}(\beta)$ in contrast to the periodic bosons), the supersymmetry can also be broken [8]. Moreover, as both fermions and scalars get masses related to the temperature (due to renormalization for the latter), they decouple at high enough temperature and the theory reduces to a pure non-conformal gauge theory. We are thus considering, at large distances, three-dimensional non-supersymmetric Yang-Mills theory at zero temperature (hence the title of this section). On the contrary, at small distances $r \ll \beta$, the compactification radius of the circle turns out to be sizeable. We deal therefore with four-dimensional supersymmetric Yang-Mills theory at zero temperature and, not surprisingly, we recover Maldacena's result $(3.73)\left(U_{0} \gg U_{T}\right.$ or $\left.\epsilon \simeq 1\right)$ :

$$
r\left(U_{0}\right)=\frac{2 \tilde{R}^{2}}{U_{0}} \int_{1}^{\infty} d v \frac{1}{v^{2} \sqrt{v^{4}-1}}=\frac{\tilde{R}^{2}}{U_{0}} \frac{1}{\rho}
$$

and the Coulomb-like behaviour of the potential (3.89). We are now ready to treat, in the supergravity approach, the static potential derived from a spatial Wilson loop with the background (4.90). Following the standard procedure, the action can be rewritten as

$$
\begin{aligned}
S_{N G}[\mathcal{C}] & =\frac{Y}{\pi} \int_{0}^{r / 2} d x \sqrt{\frac{U^{4}}{\tilde{R}^{2}}+\frac{U^{4}}{U^{4}-U_{T}^{4}} U^{\prime 2}}=\frac{Y}{\pi} \int_{0}^{r / 2} d x \frac{U^{4}}{\tilde{R}^{2} U_{0}^{2}} \\
& =\frac{Y}{\pi} \int_{U_{0}}^{\infty} \frac{d U}{U^{\prime}} \frac{U^{4}}{\tilde{R}^{2} U_{0}^{2}} \underset{\left(v \equiv \frac{U}{U_{0}}\right)}{=} Y \frac{U_{0}}{\pi} \int_{1}^{\infty} d v \frac{v^{4}}{\sqrt{\left(v^{4}-1+\epsilon\right)\left(v^{4}-1\right)}}
\end{aligned}
$$

The heavy quark potential is then given by Maldacena's prescription (3.78):

$$
V_{Q \bar{Q}}^{(R)}(r)=\lim _{\substack{Y \rightarrow \infty \\ M \rightarrow \infty}} \frac{1}{Y}\left(S_{N G}-\ell M\right)
$$

where $S_{N G}$ is the regularized action (with the UV cutoff $U \leq U_{\max }$ or $v \leq U_{\max } / U_{0}$ ) and the counter-term is similar to (4.106) $\left(\ell=2 r+2 Y \underset{Y \gg r}{\simeq} 2 Y\right.$ and $\left.M=\frac{U_{\max }-U_{T}}{2 \pi}\right)$ :

$$
V_{c . t .}=-\lim _{U_{\max } \rightarrow \infty} \frac{U_{\max }-U_{T}}{\pi}
$$


We have [31]:

$$
\begin{aligned}
V_{Q \bar{Q}}^{(R)}(r) & =\frac{U_{0}}{\pi} \int_{1}^{U_{\max } / U_{0}} d v\left(\frac{v^{4}}{\sqrt{\left(v^{4}-1+\epsilon\right)\left(v^{4}-1\right)}}-1+1\right)-\frac{U_{\max }-U_{T}}{\pi} \\
& =\frac{U_{0}}{\pi} \int_{1}^{U_{\max } / U_{0}} d v\left(\frac{\left(v^{4}-1\right)+1}{\sqrt{\left(v^{4}-1+\epsilon\right)\left(v^{4}-1\right)}}-1\right)+\frac{U_{0}}{\pi} \int_{1}^{U_{\max } / U_{0}} d v-\frac{U_{\max }-U_{T}}{\pi} \\
& =\frac{U_{0}}{\pi} \int_{1}^{\infty} \frac{U_{0}}{\pi} \int_{1}^{\infty} d v\left(\sqrt{\frac{v^{4}-1}{v^{4}-1+\epsilon}}-1\right)+\frac{U_{T}-U_{0}}{\pi} \\
V_{Q \bar{Q}}^{(R)}(r) & =\frac{U_{0}^{2}}{2 \pi \tilde{R}^{2}} r+\frac{U_{0}}{\pi} \int_{1}^{\infty} d v\left(\sqrt{\frac{v^{4}-1}{v^{4}-1+\epsilon}}-1\right)+\frac{U_{T}-U_{0}}{\pi} .
\end{aligned}
$$

We are interested in the leading and subleading terms in the static potential at large quark separation, i.e. when $U_{0} \simeq U_{T}(\epsilon \ll 1)$. If we remarks that ( $i$ is the imaginary unit):

$$
\begin{gathered}
(v-1)(v+1)(v-i)(v+i)=v^{4}-1, \\
\left(v-1+\frac{\epsilon}{4}\right)\left(v+1-\frac{\epsilon}{4}\right)\left(v-i+i \frac{\epsilon}{4}\right)\left(v+i-i \frac{\epsilon}{4}\right)=v^{4}-\left(1-\frac{\epsilon}{4}\right)^{4} \underset{\epsilon \ll 1}{\simeq} v^{4}-1+\epsilon+O\left(\epsilon^{2}\right),
\end{gathered}
$$

then the inter-quark distance $r$ (4.116) can be rewritten as

$$
r\left(U_{0}\right) \simeq \frac{2 \tilde{R}^{2}}{U_{0}} \int_{1}^{\infty} \frac{d v}{\sqrt{(v-1)\left(v-1+\frac{\epsilon}{4}\right)}} \frac{1}{\sqrt{F_{\epsilon}(v)}}
$$

where we have defined a new function:

$$
F_{\epsilon}(v)=(v+1)(v-i)(v+i)\left(v+1-\frac{\epsilon}{4}\right)\left(v-i+i \frac{\epsilon}{4}\right)\left(v+i-i \frac{\epsilon}{4}\right),
$$

regular in $y=1$ and/or $\epsilon=0$ :

$$
\begin{aligned}
& F_{\epsilon}(1)=(8-\epsilon)\left(2-\frac{\epsilon}{2}+\frac{\epsilon^{2}}{16}\right) \underset{\epsilon \ll 1}{=} 16-6 \epsilon+O\left(\epsilon^{2}\right), \\
& F_{0}(v)=(v+1)^{2}\left(v^{2}+1\right)^{2} \underset{v=1}{=} 16
\end{aligned}
$$

and which behaves asymptotically as $F_{\epsilon}(v) \underset{v \gg 1}{\sim} v^{6}$. In this way, we focus on the main contribution of the integral (4.125) which comes from the region $v=1$ (see Eq.(4.117)). A partial integration gives:

$$
\begin{aligned}
r\left(U_{0}\right) \simeq & \frac{4 \tilde{R}^{2}}{U_{0}}\left[\ln \left(\sqrt{v-1}+\sqrt{v-1+\frac{\epsilon}{4}}\right) \frac{1}{\sqrt{F_{\epsilon}(v)}}\right]_{1}^{\infty} \\
& +\frac{4 \tilde{R}^{2}}{U_{0}} \int_{1}^{\infty} d v \ln \left(\sqrt{v-1}+\sqrt{v-1+\frac{\epsilon}{4}}\right) \frac{F_{\epsilon}^{\prime}(v)}{F_{\epsilon}(v)^{3 / 2}} \\
r\left(U_{0}\right) \simeq & -\frac{\tilde{R}^{2}}{2 U_{0}} \ln \epsilon+O(\epsilon \ln \epsilon)
\end{aligned}
$$


or $\left(U_{0} \simeq U_{T}\right)$

$$
\epsilon \simeq e^{-\frac{2 U_{T}}{\tilde{R}^{2}} r} .
$$

As for the heavy quark potential (4.122), it is convenient to defined the function $J(\epsilon)$ :

$$
\begin{aligned}
J(\epsilon) & \equiv \int_{1}^{\infty} d v\left(\sqrt{\frac{v^{4}-1}{v^{4}-1+\epsilon}}-1\right) \text { with } J(0)=0, \\
\frac{\partial J(\epsilon)}{\partial \epsilon} & =-\frac{1}{2} \int_{1}^{\infty} d v \frac{\sqrt{v^{4}-1}}{\left(v^{4}-1+\epsilon\right)^{3 / 2}} .
\end{aligned}
$$

Because of (4.123) and (4.124), the integral (4.132) can be rewritten, in the limit $\epsilon \ll 1$, as

$$
\frac{\partial J(\epsilon)}{\partial \epsilon} \simeq-\frac{1}{2} \int_{1}^{\infty} d v \frac{\sqrt{v-1}}{\left(v-1+\frac{\epsilon}{4}\right)^{3 / 2}} \phi_{\epsilon}(v)
$$

where the function:

$$
\phi_{\epsilon}(v)=\frac{\sqrt{(v+1)(v-i)(v+i)}}{\left(v+1-\frac{\epsilon}{4}\right)^{3 / 2}\left(v-i+i \frac{\epsilon}{4}\right)^{3 / 2}\left(v+i-i \frac{\epsilon}{4}\right)^{3 / 2}}
$$

is regular in $y=1$ and/or $\epsilon=0$ :

$$
\begin{aligned}
& \phi_{\epsilon}(1)=\frac{2}{\left(2-\frac{\epsilon}{4}\right)^{3}} \underset{\epsilon \ll 1}{\bar{k}} \frac{1}{4}+\frac{3}{16} \epsilon+O\left(\epsilon^{2}\right), \\
& \phi_{0}(v)=\frac{1}{(v+1)\left(v^{2}+1\right)}=\frac{1}{v=1}
\end{aligned}
$$

with the asymptotic behaviour $\phi_{\epsilon}(v) \underset{v \gg 1}{\sim} \frac{1}{v^{3}}$. By partial integration, we obtain:

$$
\begin{aligned}
\frac{\partial J(\epsilon)}{\partial \epsilon} \simeq & \left\{\left[\sqrt{\frac{v-1}{v-1+\frac{\epsilon}{4}}}-\ln \left(\sqrt{v-1}+\sqrt{v-1+\frac{\epsilon}{4}}\right)\right] \phi_{\epsilon}(v)\right\}_{1}^{\infty} \\
& +\int_{1}^{\infty} d v\left[-\sqrt{\frac{v-1}{v-1+\frac{\epsilon}{4}}}+\ln \left(\sqrt{v-1}+\sqrt{v-1+\frac{\epsilon}{4}}\right)\right] \phi_{\epsilon}^{\prime}(v) \\
\frac{\partial J(\epsilon)}{\partial \epsilon} \simeq & \frac{1}{8} \ln \epsilon+I(\epsilon)+O\left(\epsilon^{0}\right) .
\end{aligned}
$$

The integral $I(\epsilon)$ can then be treated analogously:

$$
\begin{aligned}
I(\epsilon) \equiv & \int_{1}^{\infty} d v\left[-\sqrt{\frac{v-1}{v-1+\frac{\epsilon}{4}}}+\ln \left(\sqrt{v-1}+\sqrt{v-1+\frac{\epsilon}{4}}\right)\right] \phi_{\epsilon}^{\prime}(v) \\
= & \left\{\left[-\frac{3}{2} \sqrt{(v-1)\left(v-1+\frac{\epsilon}{4}\right)}+\left(v-1+\frac{3 \epsilon}{8}\right) \ln \left(\sqrt{v-1}+\sqrt{v-1+\frac{\epsilon}{4}}\right)\right] \phi_{\epsilon}^{\prime}(v)\right\}_{1}^{\infty} \\
& +\int_{1}^{\infty} d v\left[\frac{3}{2} \sqrt{(v-1)\left(v-1+\frac{\epsilon}{4}\right)}-\left(v-1+\frac{3 \epsilon}{8}\right) \ln \left(\sqrt{v-1}+\sqrt{v-1+\frac{\epsilon}{4}}\right)\right] \phi_{\epsilon}^{\prime \prime}(v)
\end{aligned}
$$


which shows, with $\phi_{\epsilon}^{\prime}(1) \underset{\epsilon \ll 1}{\overline{=}}-\frac{3}{8}-\frac{21}{64} \epsilon+O\left(\epsilon^{2}\right)$ and $\phi_{\epsilon}^{\prime}(v) \underset{v \gg 1}{\sim} \frac{1}{v^{5}}$, that $I(\epsilon)$ is of order $O(\epsilon \ln \epsilon)$ :

$$
\begin{aligned}
I(\epsilon)= & -\frac{3 \epsilon}{16} \ln \left(\frac{\epsilon}{4}\right) \phi_{\epsilon}^{\prime}(1) \\
& +\int_{1}^{\infty} d v\left[\frac{3}{2} \sqrt{(v-1)\left(v-1+\frac{\epsilon}{4}\right)}-\left(v-1+\frac{3 \epsilon}{8}\right) \ln \left(\sqrt{v-1}+\sqrt{v-1+\frac{\epsilon}{4}}\right)\right] \phi_{\epsilon}^{\prime \prime}(v) \\
I(\epsilon)= & \frac{9}{128} \epsilon \ln \epsilon+O\left(\epsilon^{2} \ln \epsilon\right) .
\end{aligned}
$$

At the end of the day, the main contribution of (4.132) in the limit $\epsilon \ll 1$ turns out to be

$$
\frac{\partial J(\epsilon)}{\partial \epsilon}=\frac{1}{8} \ln \epsilon+O(\epsilon \ln \epsilon)
$$

that is

$$
J(\epsilon)=\int_{0}^{\epsilon} \partial \epsilon^{\prime} \frac{\partial J\left(\epsilon^{\prime}\right)}{d \epsilon^{\prime}}=\frac{1}{8} \epsilon \ln (\epsilon)+O\left(\epsilon^{2} \ln \epsilon\right) .
$$

Thus, the heavy quark potential $\left(U_{0} \simeq U_{T}\right)$ :

$$
V_{Q \bar{Q}}^{(R)}\left(r, U_{T}\right)=\frac{U_{T}^{2}}{2 \pi \tilde{R}^{2}} r+\frac{U_{T}}{\pi} \frac{1}{8} \epsilon \ln (\epsilon)+O\left(\epsilon^{2} \ln \epsilon\right) .
$$

In terms of the distance between quarks (4.130), we find a leading correction to the linear potential exponentially small for $r T \gg 1$ [31]:

$$
V_{Q \bar{Q}}^{(R)}\left(r, U_{T}\right) \simeq \frac{U_{T}^{2}}{2 \pi \tilde{R}^{2}} r\left(1-\frac{1}{2} e^{-\frac{2 U_{T}}{\tilde{R}^{2}} r}\right) .
$$

On the other hand, as expected, the string tension is proportional to (the square of) the temperature since it is our only dimensionful parameter at hand:

$$
\sigma=\frac{U_{T}^{2}}{2 \pi \tilde{R}^{2}}=\frac{1}{2} \pi \tilde{R}^{2} T^{2}=\sqrt{\pi^{3} g_{s} N} T^{2} .
$$

The subleading term in the static potential at large quark separation is not in $1 / r$ which is, at first sight, in contradiction with predictions from effective string models and Lattice QCD [33]. Instead, these latter tend to confirm a subleading attractive Coulomblike contribution to the linear potential, the so-called Lüscher term $-c / r$ where $c$ is a universal numerical constant [34]. Nevertheless, this result is not so surprising since the limits at work in the supergravity approach are the large $N$ and the large 't Hooft coupling constant limits and it is known that there is no Lüscher term in the strong coupling regime on the lattice whereas it appears in the weak coupling phase. [31, 35] stressed the fact that such a phase transition could also occur in the supergravity approach as the 't Hooft coupling is reduced and argued that the Lüscher term could arise from quantum fluctuations of the classical world-sheet approximation [36]. 
Finally, it is worth pointing out that the linear behaviour of the static potential is not spoilt by the leading stringy corrections $O\left(\alpha^{\prime 3}\right)$ (which consists also of an expansion in $1 / \sqrt{N}$ according to (3.50)) of the Schwarzschild black hole- $A d S_{5}$ metric (4.90). The line element was found to be [28]:

$$
d s_{B H}^{2}=\alpha^{\prime}\left\{\left(1+\delta_{2}\right) \frac{u^{2}}{\tilde{R}^{2}} f(u) d t^{2}+\frac{u^{2}}{\tilde{R}^{2}} \sum_{i=1}^{3} d x_{i}^{2}+\left(1+\delta_{1}\right) \frac{\tilde{R}^{2}}{u^{2}} \frac{d u^{2}}{f(u)}+\tilde{R}^{2} d \Omega_{5}\right\}
$$

with the correction coefficients:

$$
\begin{aligned}
& \delta_{1}=-\frac{15}{8} \zeta(3) \alpha^{\prime 3}\left[5\left(\frac{u_{T}}{u}\right)^{4}+5\left(\frac{u_{T}}{u}\right)^{8}-3\left(\frac{u_{T}}{u}\right)^{12}\right], \\
& \delta_{2}=\frac{15}{8} \zeta(3) \alpha^{\prime 3}\left[5\left(\frac{u_{T}}{u}\right)^{4}+5\left(\frac{u_{T}}{u}\right)^{8}-19\left(\frac{u_{T}}{u}\right)^{12}\right] .
\end{aligned}
$$

The classical action of the space-like Nambu-Goto string takes then the following form:

$$
S_{N G}[\mathcal{C}]=\frac{Y}{2 \pi} \int_{-r / 2}^{r / 2} d x \sqrt{\frac{U^{4}}{\tilde{R}^{4}}+\left(1+\delta_{1}\right) \frac{U^{4}}{U^{4}-U_{T}^{4}} U^{\prime 2}}
$$

from which can be derived the inter-quark separation $r$ and the (renormalized) static potential $V_{Q \bar{Q}}^{(R)}(r)$ :

$$
\begin{aligned}
r\left(U_{0}, U_{T}\right) & =\frac{2 \tilde{R}^{2}}{U_{0}} \int_{0}^{\infty} d v \frac{\sqrt{1+\delta_{1}}}{\sqrt{\left(v^{4}-1+\epsilon\right)\left(v^{4}-1\right)}} \\
V_{Q \bar{Q}}^{(R)}(r) & =\frac{U_{0}}{2 \pi \tilde{R}^{2}} r+\frac{U_{0}}{\pi} \int_{1}^{\infty} d v\left(\sqrt{1+\delta_{1}} \sqrt{\frac{v^{4}-1}{v^{4}-1+\epsilon}}-1\right)+\frac{U_{T}-U_{0}}{\pi}
\end{aligned}
$$

The integrals are modified only by terms in $1 / v$ which do not rule out the logarithmic singularity in $v=1$ in the limit $U_{0} \simeq U_{T}$ (see Eq.(4.117)).

\subsection{The area law in four-dimensional Yang-Mills theory}

We have seen previously that the Schwarzschild black hole- $A d S_{5}$ geometry was required in order to deal with a three-dimensional gauge theory (after compactification of the Euclidean time direction). If we are interested in studying higher-dimensional gauge theories, it is then necessary to consider the general case of a stack of $N$ coincident (extremal i.e. without horizon) $D p$-branes in the decoupling limit. We are therefore led to the (Euclidean) metric [37]:

$$
d s^{2}=\alpha^{\prime}\left\{\frac{u^{\frac{(7-p)}{2}}}{g_{Y M}^{(p+1)} \sqrt{d_{p} N}}\left(d t^{2}+\sum_{i=1}^{p} d x_{i}^{2}\right)+\frac{g_{Y M}^{(p+1)} \sqrt{d_{p} N}}{u^{\frac{7-p}{2}}} d u^{2}+g_{Y M}^{(p+1)} \sqrt{d_{p} N} u^{\frac{(p-3)}{2}} d \Omega_{8-p}^{2}\right\}
$$


with $d_{p} \equiv 2^{7-2 p} \pi^{\frac{9-3 p}{2}} \Gamma\left(\frac{7-p}{2}\right)$. The coupling constant $g_{Y M}^{(p+1)}$ of the $(p+1)$-dimensional $S U(N)$ super-Yang-Mills theory defined on the world-volume of the $N D p$-branes is related to the closed string coupling constant $g_{s}$ as follows:

$$
g_{Y M}^{(p+1)^{2}}=(2 \pi)^{p-2} g_{s} \alpha^{\frac{(p-3)}{2}} .
$$

The case of interest here consists of $p=4$ for which $g_{Y M}^{(5)}{ }^{2}=4 \pi^{2} g_{s} \sqrt{\alpha^{\prime}}$ (hence, $g_{Y M}^{(5)}$ has the dimension of a (length) $)^{1 / 2}$ ) and the metric (4.151) becomes:

$$
d s^{2}=\alpha^{\prime}\left\{\frac{u^{3 / 2}}{R_{4}^{3 / 2}}\left(d t^{2}+\sum_{i=1}^{4} d x_{i}^{2}\right)+\frac{R_{4}^{3 / 2}}{u^{3 / 2}} d u^{2}+R_{4}^{3 / 2} \sqrt{u} d \Omega_{4}^{2}\right\} .
$$

We have defined $R_{4}^{3 / 2} \equiv g_{Y M}^{(5)} \sqrt{d_{4} N}=g_{Y M}^{(5)} \sqrt{\frac{N}{4 \pi}}$ such that $R_{4}$ has the dimension of a $(\text { length })^{1 / 3}$. When one turns on the temperature, the metric is solution of the equations of motion for a stack of $N$ coincident (non-extremal i.e in the presence of a horizon) $D p$-branes in the decoupling limit:

$$
d s_{B H}^{2}=\alpha^{\prime}\left\{\frac{u^{3 / 2}}{R_{4}^{3 / 2}}\left(g(u) d t^{2}+\sum_{i=1}^{4} d x_{i}^{2}\right)+\frac{R_{4}^{3 / 2}}{u^{3 / 2}} \frac{d u^{2}}{g(u)}+R_{4}^{3 / 2} \sqrt{u} d \Omega_{4}^{2}\right\}
$$

where

$$
g(u)=1-\frac{u_{T}^{3}}{u^{3}}
$$

The event horizon at $u_{T}$ is given in terms of the Beckenstein-Hawking temperature $T$ :

$$
T=\left.\frac{1}{4 \pi \alpha^{\prime}} \frac{\partial \delta_{00}}{\partial u}\right|_{u=u_{T}}=\frac{3}{4 \pi R_{4}^{3 / 2}} \sqrt{u_{T}}
$$

which gives:

$$
u_{T}=\frac{16}{9} \pi^{2} R_{4}^{3} T^{2}=\frac{4}{9} \pi g_{Y M}^{(5)}{ }^{2} N T^{2} .
$$

The Nambu-Goto action (3.58) of the space-like string world-sheet is this time:

$$
S_{N G}=\frac{Y}{2 \pi} \int_{-r / 2}^{r / 2} d x \sqrt{\frac{U^{3}}{R_{4}^{3}}+\frac{U^{3}}{U^{3}-U_{T}^{3}} U^{\prime 2}}
$$

and the integral expressions for the distance $r$ between the quarks and the static potential are $\left(\epsilon \equiv g\left(U_{0}\right)=1-\frac{U_{T}^{3}}{U_{0}^{3}}\right)$ :

$$
\begin{aligned}
r\left(U_{0}, U_{T}\right) & =\frac{2 R_{4}^{3 / 2}}{U_{0}^{1 / 2}} \int_{1}^{\infty} \frac{d v}{\sqrt{\left(v^{3}-1+\epsilon\right)\left(v^{3}-1\right)}}, \\
V_{Q \bar{Q}}^{(R)}(r) & =\frac{U_{0}^{3 / 2}}{2 \pi R_{4}^{3 / 2}} r+\frac{U_{0}}{\pi} \int_{1}^{\infty} d v\left(\sqrt{\frac{v^{3}-1}{v^{3}-1+\epsilon}}-1\right)+\frac{U_{T}-U_{0}}{\pi} .
\end{aligned}
$$


Also, we find that the potential presents an area law behaviour in the case of a fourdimensional non-supersymmetric gauge theory with a string tension $\left(U_{0} \simeq U_{T}\right)$ :

$$
\sigma=\frac{U_{T}^{3 / 2}}{2 \pi R_{4}^{3 / 2}}=\frac{8}{27} \pi g_{Y M}^{(4)}{ }^{2} N T^{2}
$$

expressed in terms of the dimensionless coupling constant $g_{Y M}^{(4)}$ of the four-dimensional gauge theory [32]. This latter is obtained from $g_{Y M}^{(5)}$ after compactification of the Euclidean time direction along a circle $S^{1}(\beta)$ of circumference $\beta$. We have indeed:

$$
\int d^{5} x \frac{1}{g_{Y M}^{(5)^{2}}}=\int d^{4} x \frac{\beta}{g_{Y M}^{(5)^{2}}}=\int d^{4} x \frac{1}{g_{Y M}^{(4)^{2}}}
$$

namely, $g_{Y M}^{(4)}{ }^{2}=g_{Y M}^{(5)}{ }^{2} T$.

To summarize, we observe an area law for spatial Wilson loops in four- and fivedimensional supersymmetric Yang-Mills theories at finite temperature. This can be interpreted as the area law of ordinary Wilson loops (after having identified one of the spatial coordinates of the higher-dimensional theory as the non-compactified Euclidean time) in three- and four-dimensional non-supersymmetric Yang-Mills theories at zero temperature which indicates confinement in these theories.

\section{The heavy quark potential in holographic models of QCD}

\subsection{Andreev and Zakharov's model}

The holographic models of QCD imply to introduce a dimensionful parameter related in some way to the QCD mass gap. This can be the cutoff $z_{m}$ where is located the IR brane in the Hard Wall Model [9, 10, 11] or the dilaton parameter in the Soft Wall Model [17]. In $[38,39]$, the authors chose to break the isometry group of the holographic space-time $A d S_{5}$ (i.e. the conformal invariance of the boundary field theory) by means of a warp factor $h(z)$ in the Euclidean metric:

$$
d s^{2}=g_{M N}(x) d x^{M} d x^{N}=\frac{R^{2}}{z^{2}} h(z) \delta_{M N} d x^{M} d x^{N} .
$$

The bulk coordinates are $x^{M}=\left(x^{\mu}, z\right)$ with $x^{\mu}(\mu=0, \ldots, 3)$ the boundary coordinates and $z>0$ the holographic coordinate. $\delta_{M N}=\operatorname{diag}(+1,+1,+1,+1,+1)$ is the Euclidean flat metric tensor. In this model, the warp factor $h(z) \equiv e^{\frac{1}{2} c z^{2}}$ introduces the conformal symmetry breaking parameter $c$ and we recover the $A d S_{5}$ metric (3.54) near the UV 
brane $z \rightarrow 0$ where $h(0)=1$. It is worth pointing out that this $c$ does not have to be identified with the (square of the) dilaton parameter $\Phi(z)=c_{\Phi}^{2} z^{2}$ in the IR Soft Wall approximation. For example, even if the equivalence holds for the vector meson case [17] (and then $c_{\Phi}^{2}=\frac{c}{4}$ ), it is not true in general (see, for instance, the effective action for the scalar mesons [19]).

As usual, we start from the Nambu-Goto action (3.58) in the static gauge (3.60)(3.61) $X^{0}(\tau, \sigma)=\tau$ with $\sigma=x$. The non-vanishing components of the induced metric $\gamma_{a b}$ $(a, b=1,2)$ on the world-sheet (3.59) are:

$$
\begin{aligned}
& \gamma_{11}=\frac{R^{2}}{z^{2}} h(z) \\
& \gamma_{22}=\frac{R^{2}}{z^{2}} h(z)\left(1+z^{\prime 2}\right)
\end{aligned}
$$

where the holographic coordinate of the string $z(x)$ is a function only of $x$ in the limit $T \rightarrow \infty$ (in order to not overweight the notation, we give up the convention of writing the string coordinates with capital letters). The Nambu-Goto action of the string is then (the notation $g$ instead of $\tilde{R}^{2}$ is used in [39]):

$$
S_{N G}[\mathcal{C}]=\frac{\tilde{R}^{2}}{2 \pi} T \int_{-\frac{r}{2}}^{\frac{r}{2}} d x \frac{h}{z^{2}} \sqrt{1+z^{\prime 2}}
$$

where $\mathcal{C}$ is the rectangular loop already considered in (3.52)-(3.53). The equation of motion for $z(x)$ and the first integral read respectively as

$$
\delta_{z} S_{N G}=0 \Rightarrow z z^{\prime \prime}+\left(2-c z^{2}\right)\left(1+z^{\prime 2}\right)=0
$$

and

$$
\frac{h}{z^{2} \sqrt{1+\left(z^{\prime}\right)^{2}}}=C \text {. }
$$

The integration constant $C$ is positive and can be evaluated for any value of $z(x)$. Especially, at $x=0$, we have $z(0) \equiv z_{0}$ and $z^{\prime}(0)=0$ by symmetry such that $C=\frac{e^{\frac{1}{2} \lambda}}{z_{0}^{2}}$ where we have defined:

$$
\lambda \equiv c z_{0}^{2}
$$

The two parametric expressions for the inter-quark distance $r\left(z_{0}, c\right)$ and the interaction potential $V\left(z_{0}, c\right)$ take the following forms:

$$
\begin{aligned}
& r\left(z_{0}, c\right)=2 \int_{0}^{\frac{r}{2}} d x=2 \int_{z_{0}}^{0} \frac{d z}{z^{\prime}}=2 \int_{0}^{z_{0}} d z \frac{C z^{2}}{h}\left(1-\frac{C^{2} z^{4}}{h^{2}}\right)^{-\frac{1}{2}} \\
&=2 C \int_{0}^{z_{0}} d z z^{2} e^{-\frac{1}{2} c z^{2}}\left(1-\frac{z^{4}}{z_{0}^{4}} e^{\lambda-c z^{2}}\right)^{-\frac{1}{2}} \\
& r(\lambda, c) \underset{\substack{\left(v=\frac{z}{z_{0}}\right) \\
=}}{2} \sqrt{\frac{\lambda}{c}} \int_{0}^{1} d v v^{2} e^{\frac{1}{2} \lambda\left(1-v^{2}\right)}\left(1-v^{4} e^{\lambda\left(1-v^{2}\right)}\right)^{-\frac{1}{2}}
\end{aligned}
$$


and

$$
\begin{aligned}
& V\left(z_{0}, c\right)=\lim _{T \rightarrow \infty} \frac{1}{T} S_{N G}[\mathcal{C}]=\frac{\tilde{R}^{2}}{2 \pi} \int_{-\frac{r}{2}}^{\frac{r}{2}} d x \frac{h}{z^{2}} \sqrt{1+z^{\prime 2}} \\
&=\frac{\tilde{R}^{2}}{\pi} \int_{z_{0}}^{0} \frac{d z}{z^{\prime}} \frac{h}{z^{2}} \sqrt{1+z^{\prime 2}}=\frac{\tilde{R}^{2}}{\pi} \int_{0}^{z_{0}} d z \frac{h}{z^{2}}\left(1-\frac{C^{2} z^{4}}{h^{2}}\right)^{-\frac{1}{2}} \\
& V(\lambda, c) \underset{\substack{\left(v=\frac{z}{z_{0}}\right) \\
=}}{ } \frac{\tilde{R}^{2}}{\pi} \sqrt{\frac{c}{\lambda}} \int_{0}^{1} d v \frac{e^{\frac{1}{2} \lambda v^{2}}}{v^{2}}\left(1-v^{4} e^{\lambda\left(1-v^{2}\right)}\right)^{-\frac{1}{2}}
\end{aligned}
$$

where we have made use of the expression of $z^{\prime}(x)$ derived from the first integral (5.167):

$$
z^{\prime}(x)= \pm \frac{h}{C z^{2}}\left(1-\frac{C^{2} z^{4}}{h^{2}}\right)^{\frac{1}{2}} .
$$

The plus (minus) sign corresponds to $-\frac{r}{2}<x<0\left(0<x<\frac{r}{2}\right)$. As expected from our previous studies (see, e.g., the Eqs.(3.77) and (4.119)), the integral (5.170) does not converge when $v \rightarrow 0$ and require an UV cutoff $z(x) \geq z_{\text {min }}$ :

$$
\begin{aligned}
V^{(\text {reg. })}\left(\lambda, c, z_{\text {min }}\right) & =\frac{\tilde{R}^{2}}{\pi} \sqrt{\frac{c}{\lambda}} \int_{0}^{1} \frac{d v}{v^{2}}\left[e^{\frac{1}{2} \lambda v^{2}}\left(1-v^{4} e^{\lambda\left(1-v^{2}\right)}\right)^{-\frac{1}{2}}-1\right]+\frac{\tilde{R}^{2}}{\pi} \sqrt{\frac{c}{\lambda}} \int_{z_{\min } / z_{0}}^{1} \frac{d v}{v^{2}} \\
& =\frac{\tilde{R}^{2}}{\pi} \sqrt{\frac{c}{\lambda}}\left\{-1+\int_{0}^{1} \frac{d v}{v^{2}}\left[e^{\frac{1}{2} \lambda v^{2}}\left(1-v^{4} e^{\lambda\left(1-v^{2}\right)}\right)^{-\frac{1}{2}}-1\right]\right\}+\frac{\tilde{R}^{2}}{\pi} \frac{1}{z_{\text {min }}} .
\end{aligned}
$$

If one remembers the relation $U=\frac{\tilde{R}^{2}}{z}(3.56)$ between the two holographic coordinates $z$ and $U$, then the last term on the r.h.s. of (5.172) is canceled out, according to Maldacena's prescription, by the same counter-term present in (3.83). At the end of the day, in a holographic space-time with the background metric (5.163), the renormalized (or subtracted) potential is:

$$
V^{(R)}(\lambda, c)=\frac{\tilde{R}^{2}}{\pi} \sqrt{\frac{c}{\lambda}}\left\{-1+\int_{0}^{1} \frac{d v}{v^{2}}\left[e^{\frac{1}{2} \lambda v^{2}}\left(1-v^{4} e^{\lambda\left(1-v^{2}\right)}\right)^{-\frac{1}{2}}-1\right]\right\} .
$$

\subsubsection{The heavy quark potential at large distances}

As a matter of fact, the expression (5.169) has a logarithmic singularity when $\lambda=c z_{0}^{2}=2$. This peculiar finite value of $z_{0}=\sqrt{\frac{2}{c}}$ corresponds to the maximal extent reached by the string world-sheet along the holographic coordinate. There, the inter-quark distance $r(\lambda, c)$ explodes, which mimics the confinement mechanism. On the contrary, if the conformal symmetry breaking parameter $c=0$, then $z_{0}$ is allowed to run over all the holographic dimension $\left(0<z_{0}<\infty\right)$ and we do not have confinement anymore. Let us identify this logarithmic singularity. Since $r(2, c)$ does not converge, it is not allowed to expand (5.169) 
in powers of $(2-\lambda)$. Nevertheless, as we are interested in the region $z \sim z_{0}$, i.e. $v \sim 1$, the integral in $r(\lambda, c)$ can be approximately replaced by its main contribution:

$$
r(\lambda, c) \simeq 2 \sqrt{\frac{\lambda}{c}} \int_{0}^{1} \frac{d v}{\sqrt{2(2-\lambda)(1-v)+\left(-2 \lambda^{2}+9 \lambda-6\right)(1-v)^{2}}} .
$$

The quark separation has clearly a logarithmic singularity at $\lambda=2$ :

$$
r(\lambda, c) \underset{\substack{\lambda=2 \\ v \rightarrow 1}}{\sim}-\sqrt{\frac{2}{c}} \ln (1-v)
$$

The static potential (5.173) develops the same singularity when $v \rightarrow 1$ at $\lambda=2$. Indeed, we can write:

$$
V^{(R)}(\lambda, c) \simeq \frac{\tilde{R}^{2}}{\pi} \sqrt{\frac{c}{\lambda}}\left\{-1+\int_{0}^{1} d v\left[\frac{e}{\sqrt{2(2-\lambda)(1-v)+\left(-2 \lambda^{2}+9 \lambda-6\right)(1-v)^{2}}}-1\right]\right\}
$$

where $e$ is the exponential function of the unit. At large distances, we have then:

$$
V^{(R)}(\lambda, c) \underset{\substack{\lambda=2 \\ v \rightarrow 1}}{\sim}-\frac{\tilde{R}^{2}}{2 \pi} \sqrt{\frac{c}{2}} e \ln (1-v)
$$

which gives, in terms of $r$, a linear confining potential:

$$
V^{(R)}(r, c)=\sigma r
$$

where we have defined the large-distance string tension:

$$
\sigma=\tilde{R}^{2} \frac{e}{4 \pi} c
$$

\subsubsection{The heavy quark potential at short distances}

The behaviours of $r(\lambda, c)$ and $V^{(R)}(\lambda, c)$ at short distances correspond to a string configuration with $z_{0} \sim 0$, namely to the limit $\lambda \rightarrow 0$ (since then the string world-sheet does not go far away along the fifth holographic coordinate, it mainly feels the UV geometry of the background metric. As a consequence, there is no IR correction to the potential [39]). The expansion of the inter-quark distance in power series up to the order $O\left(\lambda^{2}\right)$ yields:

$$
\begin{aligned}
r(\lambda, c) & =2 \sqrt{\frac{\lambda}{c}} \int_{0}^{1} d v \frac{v^{2}}{\sqrt{1-v^{4}}}\left(1+\frac{\lambda}{2} \frac{\left(1-v^{2}\right)}{\left(1-v^{4}\right)}+O\left(\lambda^{2}\right)\right) \\
& =2 \sqrt{\frac{\lambda}{c}} \int_{0}^{1} d v \frac{v^{2}}{\sqrt{1-v^{4}}}+\lambda \sqrt{\frac{\lambda}{c}} \int_{0}^{1} d v\left(\frac{v^{2}}{\left(1-v^{4}\right)^{3 / 2}}-\frac{v^{4}}{\left(1-v^{4}\right)^{3 / 2}}\right)+O\left(\lambda^{5 / 2}\right) .
\end{aligned}
$$


The first integral on the r.h.s. gives the well-known $A d S / \mathrm{CFT}$ result (3.73):

$$
r(\lambda, c)=\sqrt{\frac{\lambda}{c}} \frac{1}{\rho}+O\left(\lambda^{3 / 2}\right)
$$

with $\rho=\frac{\Gamma(1 / 4)^{2}}{(2 \pi)^{3 / 2}}$ the usual numerical factor and $\sqrt{\frac{\lambda}{c}}=z_{0}=\frac{\tilde{R}^{2}}{U_{0}}$. Although the second and the third integrals are singular when $v \rightarrow 1$, in fact their divergences $\sim 1 / \sqrt{1-v^{4}}$ cancel out each other. This can be easily seen as follows:

$$
\begin{aligned}
r(\lambda, c) & =\sqrt{\frac{\lambda}{c}}\left[\frac{1}{\rho}+\lambda \int_{0}^{1} d v\left(-\frac{\left(1-v^{2}\right)-1}{\left(1-v^{4}\right)^{3 / 2}}+\frac{\left(1-v^{4}\right)-1}{\left(1-v^{4}\right)^{3 / 2}}\right)+O\left(\lambda^{2}\right)\right] \\
& =\sqrt{\frac{\lambda}{c}}\left[\frac{1}{\rho}+\lambda \int_{0}^{1} d v\left(-\frac{1}{\sqrt{1-v^{2}}\left(1+v^{2}\right)^{3 / 2}}+\frac{1}{\sqrt{1-v^{4}}}\right)+O\left(\lambda^{2}\right)\right] \\
r(\lambda, c) & =\sqrt{\frac{\lambda}{c}}\left[\frac{1}{\rho}+\lambda\left(-\frac{1}{2} E(-1)+\frac{\sqrt{\pi} \Gamma(5 / 4)}{\Gamma(3 / 4)}\right)+O\left(\lambda^{2}\right)\right]
\end{aligned}
$$

where $E(-1)$ is the complete Elliptic integral of second kind (3.87). By standard handling of the Gamma functions (such that the formulae $x \Gamma(x)=\Gamma(x+1)$ and $\Gamma(x) \Gamma(1-x)=$ $\left.\frac{\pi}{\sin (\pi x)}\right)$, we finally obtain:

$$
r(\lambda, c)=\sqrt{\frac{\lambda}{c}} \frac{1}{\rho}\left(1-\frac{\lambda}{4}\left(1-\pi \rho^{2}\right)+O\left(\lambda^{2}\right)\right) .
$$

Furthermore, it will be worthwhile to express $\lambda$ in terms of $r$ when we will attempt to write the potential $V^{(R)}(r, c)$. Successive iterations give then:

$$
\sqrt{\frac{\lambda}{c}}=\rho r\left(1+\frac{\lambda}{4}\left(1-\pi \rho^{2}\right)+O\left(\lambda^{2}\right)\right)=\rho r\left(1+\frac{c}{4} \rho^{2} r^{2}\left(1-\pi \rho^{2}\right)+O\left(r^{4}\right)\right)
$$

since

$$
\lambda=c \rho^{2} r^{2}\left(1+\frac{\lambda}{2}\left(1-\pi \rho^{2}\right)+O\left(\lambda^{2}\right)\right)=c \rho^{2} r^{2}\left(1+O\left(r^{2}\right)\right) .
$$

The heavy quark potential (5.173) is treated in the same way as the inter-quark distance. We get:

$$
\begin{aligned}
V^{(R)}(\lambda, c) & =\frac{\tilde{R}^{2}}{\pi} \sqrt{\frac{c}{\lambda}}\left\{-1+\int_{0}^{1} \frac{d v}{v^{2}}\left[\frac{1}{\sqrt{1-v^{4}}}-1\right]+\frac{\lambda}{2} \int_{0}^{1} d v \frac{1+v^{2}-2 v^{4}}{\left(1-v^{4}\right)^{3 / 2}}+O\left(\lambda^{2}\right)\right\} \\
& =\frac{\tilde{R}^{2}}{\pi} \sqrt{\frac{c}{\lambda}}\left\{-\frac{1}{2 \rho}+\frac{\lambda}{16 \sqrt{2 \pi}}[12 \Gamma(1 / 4) \Gamma(5 / 4)+\Gamma(3 / 4) \Gamma(-1 / 4)]+O\left(\lambda^{2}\right)\right\} \\
V^{(R)}(\lambda, c) & =-\frac{\tilde{R}^{2}}{\pi} \sqrt{\frac{c}{\lambda}} \frac{1}{2 \rho}\left(1+\frac{\lambda}{4}\left(1-3 \pi \rho^{2}\right)+O\left(\lambda^{2}\right)\right)
\end{aligned}
$$

where the two first contributions in the first line consist of the renormalized expression (3.83) of the $A d S / C F T$ potential (after appropriate variable changes). We are now able 
to write the potential $V^{(R)}(r, c)$ as a function of the distance $r$ between the quarks:

$$
\begin{aligned}
V^{(R)}(r) & =-\frac{\tilde{R}^{2}}{2 \pi \rho^{2}} \frac{1}{r}\left(1-\frac{c}{4} \rho^{2} r^{2}\left(1-\pi \rho^{2}\right)+O\left(r^{4}\right)\right)-\frac{\tilde{R}^{2}}{8 \pi}\left(1-3 \pi \rho^{2}\right) c r\left(1+O\left(r^{2}\right)\right) \\
& =-\frac{\kappa_{0}}{r}+\sigma_{0} r+O\left(r^{3}\right)
\end{aligned}
$$

with

$$
\left\{\begin{array}{l}
\kappa_{0}=\frac{\tilde{R}^{2}}{2 \pi \rho^{2}}=\frac{\sqrt{4 \pi g_{s} N}}{2 \pi \rho^{2}} \\
\sigma_{0}=\tilde{R}^{2} \frac{c \rho^{2}}{4} .
\end{array}\right.
$$

Although the linear term in the Cornell potential (2.20) has only one string tension for any length scale, it appears, in the supergravity side, two tensions $\sigma(5.178)$ and $\sigma_{0}(5.188)$ corresponding respectively to the large and short distance regimes. Nevertheless, their ratio turns out to be rather closed to one:

$$
\frac{\sigma}{\sigma_{0}}=\frac{e}{\pi \rho^{2}}=\frac{8 \pi^{2} e}{\Gamma(1 / 4)^{4}} \simeq 1.24
$$

Without being obviously conclusive, this estimate is satisfactory at the accuracy level usually associated with holographic models of QCD. As for the Coulomb-like term in (5.187), it does not have to be identified with the perturbative part of the Cornell potential. This is reminiscent of what happens in the $A d S / C F T$ correspondence where the potential in $1 / r(3.89)$ behaves not as a power of the 't Hooft coupling but as the square root thereof. Nevertheless, it is hard, in the string picture used here, to disentangle the contributions, if any, of the large distance Lüscher term in $1 / r$ from the perturbative Coulomb term at short distances.

It is worth pointing out that Andreev and Zakharov's model has also been used to explore finite temperature features of a heavy quark-antiquark pair as, for instance, the spatial string tension [40] or the free energy [41]. The line element (5.163) has also been considered in [42] in order to study the baryon potential and the Y-ansatz of the baryonic area law.

\subsection{The heavy quark potential from general geometry in $A d S / Q C D$}

In the following, we will consider a general form of the metric which respects Poincaré symmetry on the boundary [43, 44]:

$$
d s^{2}=\alpha^{\prime} \tilde{R}^{2}\left(f(z) \delta_{\mu \nu} d x^{\mu} d x^{\nu}+\frac{d z^{2}}{z^{2}}\right)
$$

with $\delta_{\mu \nu}=\operatorname{diag}(+1,+1,+1,+1)$ the four-dimensional Euclidean flat metric tensor. The

warp factor $f(z)>0$ is assumed to be positive. In particular, $f(z)=\frac{1}{z^{2}}$ corresponds to the 
Euclidean $A d S_{5}$ line element. The Nambu-Goto action in the static gauge $X^{0}(\tau, \sigma)=\tau$ and $\sigma=x$ reads

$$
S_{N G}[\mathcal{C}]=\frac{\tilde{R}^{2}}{2 \pi} \int_{-T / 2}^{T / 2} d t \int_{-r / 2}^{r / 2} d x f(z) \sqrt{1+\frac{z^{\prime 2}}{f(z) z^{2}}}
$$

where $z(x)$ is the holographic coordinate of the string. The Lagrangian density does not depend explicitly on $x$ which gives us the first integral:

$$
\frac{f(z)}{\sqrt{1+\frac{z^{\prime 2}}{f(z) z^{2}}}}=f_{0}
$$

where $z_{0}$ is the value of $z(x)$ at $x=0$, that is, the maximal extent of the string world-sheet along the holographic dimension where $z^{\prime}(0)=0$ by symmetry and $f_{0} \equiv f\left(z_{0}\right)$. We derive the expression of the derivative $z^{\prime}(x)(-r / 2<x<0$ for the plus sign and $0<x<r / 2$ for the minus sign):

$$
z^{\prime}(x)= \pm z \sqrt{f(z)} \sqrt{\frac{f^{2}}{f_{0}^{2}}-1} .
$$

The equation of motion is:

$$
z z^{\prime \prime}-z^{\prime 2}-z^{3} f^{\prime}(z)-\frac{3}{2} \frac{f^{\prime}(z)}{f(z)} z z^{\prime 2}=0
$$

where $z^{\prime}(x) \equiv \frac{d z}{d x}$ and $f^{\prime}(z) \equiv \frac{d f}{d z}$ are the derivatives with respect to the arguments. In the anti-de Sitter case where $f(z)=\frac{1}{z^{2}}$, we recover the equation of motion (5.166) (with $c=0$ ) which describes the behaviour of the string world-sheet spreading into the $A d S_{5}$ holographic space-time.

The inter-quark distance takes the general form:

$$
\begin{aligned}
r\left(z_{0}\right) & =\int_{-r / 2}^{r / 2} d x=2 \int_{z_{0}}^{0} \frac{d z}{z^{\prime}}=2 \int_{0}^{z_{0}} \frac{d z}{z} \frac{1}{\sqrt{f}}\left(\frac{f^{2}}{f_{0}^{2}}-1\right)^{-\frac{1}{2}} \\
& =2 \int_{0}^{z_{0}} d z \frac{1}{\sqrt{\tilde{f}}}\left(\frac{z_{0}^{4} \tilde{f}^{2}}{z^{4} \tilde{f}_{0}^{2}}-1\right)^{-\frac{1}{2}} .
\end{aligned}
$$

Following [44], we have defined $\tilde{f}(z)=z^{2} f(z)$ such that $\tilde{f}(0)=1$. As for the interaction potential, we find successively:

$$
\begin{aligned}
V\left(z_{0}\right) & =\frac{\tilde{R}^{2}}{\pi} \int_{0}^{z_{0}} \frac{d z}{z^{2}} \sqrt{\tilde{f}}\left(1-\frac{z^{4} \tilde{f}_{0}^{2}}{z_{0}^{4} \tilde{f}^{2}}\right)^{-\frac{1}{2}} \\
V^{(\text {reg. })}\left(z_{0}, z_{\min }\right) & =\frac{\tilde{R}^{2}}{\pi}\left\{-\frac{1}{z_{0}}+\int_{0}^{z_{0}} \frac{d z}{z^{2}}\left[\sqrt{\tilde{f}}\left(1-\frac{z^{4} \tilde{f}_{0}^{2}}{z_{0}^{4} \tilde{f}^{2}}\right)^{-\frac{1}{2}}-1\right]\right\}+\frac{\tilde{R}^{2}}{\pi} \frac{1}{z_{\min }} \\
V^{(R)}\left(z_{0}\right) & =\frac{\tilde{R}^{2}}{\pi}\left\{-\frac{1}{z_{0}}+\int_{0}^{z_{0}} \frac{d z}{z^{2}}\left[\sqrt{\tilde{f}}\left(1-\frac{z^{4} \tilde{f}_{0}^{2}}{z_{0}^{4} \tilde{f}^{2}}\right)^{-\frac{1}{2}}-1\right]\right\}
\end{aligned}
$$


The last expression of the energy is obtained as usual, once the infinite contribution $\frac{\tilde{R}^{2}}{\pi} \frac{1}{z_{\min }}$ $\left(z_{\text {min }} \rightarrow 0\right)$ stemming from the "W-boson string" associated with the very massive quarks is subtracted.

On the one hand, at short distances i.e. when the string world-sheet is close enough to the boundary space-time, the bulk geometry felt by this latter is nearly $A d S_{5}$. Not surprisingly, the limit $z_{0} \rightarrow 0$ (and then $z \rightarrow 0$ and $\tilde{f}(z) \rightarrow 1$ in (5.195) and (5.196)) gives the famous $A d S / \mathrm{CFT}$ results (3.73) and (3.84):

$$
\begin{aligned}
r\left(z_{0}\right) & \underset{z_{0} \rightarrow 0}{\simeq} 2 \int_{0}^{z_{0}} d z \frac{z^{2}}{\sqrt{z_{0}^{4}-z^{4}}}=\frac{z_{0}}{\rho} \\
V^{(R)}\left(z_{0}\right) & \underset{z_{0} \rightarrow 0}{\simeq} \frac{\tilde{R}^{2}}{\pi}\left\{-\frac{1}{z_{0}}+\int_{0}^{z_{0}} \frac{d z}{z^{2}}\left[\frac{1}{\sqrt{1-\frac{z^{4}}{z_{0}^{4}}}}-1\right]\right\}=-\frac{\tilde{R}^{2}}{2 \pi \rho} \frac{1}{z_{0}}
\end{aligned}
$$

such that

$$
V^{(R)}(r)=-\frac{\tilde{R}^{2}}{2 \pi \rho^{2}} \frac{1}{r} .
$$

On the other hand, in the case of mesonic bound-states, the confinement criterion can be stated as follows: there exists a finite value $z_{0}^{*}$ of the maximal extent of the world-sheet along the holographic coordinate such that the distance $r\left(z_{0}^{*}\right)$ between quarks diverges. This peculiar value $z_{0}^{*}$ is related to the QCD mass gap. In particular, it enters the expression of the string tension in the confining linear potential. Moreover, this divergence is logarithmic. By expanding around $z_{0}^{*}$, we have indeed (where $\left.\tilde{f}\left(z_{0}^{*}\right) \equiv \tilde{f}_{0}^{*}\right)$ :

$$
\begin{aligned}
r\left(z_{0}^{*}\right)= & 2 \int_{0}^{z_{0}^{*}} d z \frac{1}{\sqrt{\tilde{f}(z)}}\left(\frac{z_{0}^{* 4} \tilde{f}^{2}(z)}{z^{4} \tilde{f}_{0}^{* 2}}-1\right)^{-\frac{1}{2}} \\
\simeq & \frac{2}{\sqrt{\tilde{f}\left(z_{0}^{*}\right)}} \int_{0}^{z_{0}^{*}} d z\left\{\left[\frac{4}{z_{0}^{*}}-\left.\frac{2}{\tilde{f}_{0}^{*}} \frac{d \tilde{f}}{d z}\right|_{z_{0}^{*}}\right]\left(z_{0}^{*}-z\right)\right. \\
& \left.+\left[\frac{10}{z_{0}^{* 2}}-\left.\frac{8}{z_{0}^{*} \tilde{f}_{0}^{*}} \frac{d \tilde{f}}{d z}\right|_{z_{0}^{*}}+\left.\frac{1}{\tilde{f}_{0}^{*}} \frac{d^{2} \tilde{f}}{d z^{2}}\right|_{z_{0}^{*}}+\frac{1}{\tilde{f}_{0}^{* 2}}\left(\left.\frac{d \tilde{f}}{d z}\right|_{z_{0}^{*}}\right)^{2}\right]\left(z_{0}^{*}-z\right)^{2}+\ldots\right\}^{-\frac{1}{2}} .
\end{aligned}
$$

With the background metric (5.190), the confinement criterion is then [44]:

$$
\left.z_{0}^{*} \frac{d \tilde{f}}{d z}\right|_{z_{0}^{*}}=2 \tilde{f}_{0}^{*}
$$

such that $r\left(z_{0}^{*}\right)$ diverges logarithmically:

$$
r\left(z_{0}^{*}\right) \underset{z \rightarrow z_{0}^{*}}{\sim}-\ln \left(1-\frac{z}{z_{0}^{*}}\right)
$$


We are also interested in the asymptotic behaviour of the potential. From our previous studies, we expect the same kind of singularity than for $r\left(z_{0}^{*}\right)$. We find indeed:

$$
\begin{aligned}
& V^{(R)}\left(z_{0}^{*}\right)=\frac{\tilde{R}^{2}}{\pi}\left\{-\frac{1}{z_{0}^{*}}+\int_{0}^{z_{0}^{*}} \frac{d z}{z^{2}}\left[\sqrt{\tilde{f}(z)}\left(1-\frac{z^{4} \tilde{f}_{0}^{* 2}}{z_{0}^{* 4} \tilde{f}^{2}(z)}\right)^{-\frac{1}{2}}-1\right]\right\} \\
& \underset{z \rightarrow z_{0}^{*}}{\simeq} \frac{\tilde{R}^{2}}{\pi} \frac{\sqrt{\tilde{f}_{0}^{*}}}{z_{0}^{* 2}} \int_{0}^{z_{0}^{*}} d z\left(\frac{z_{0}^{* 4} \tilde{f}^{2}(z)}{z^{4} \tilde{f}_{0}^{* 2}}-1\right)^{-\frac{1}{2}} .
\end{aligned}
$$

The integral is the same that enters the expression of the inter-quark distance such that

$$
V^{(R)}\left(r, z_{0}^{*}\right)=\sigma\left(z_{0}^{*}\right) r
$$

with the string tension:

$$
\sigma\left(z_{0}^{*}\right)=\frac{\tilde{R}^{2}}{2 \pi} \frac{\tilde{f}_{0}^{*}}{z_{0}^{* 2}} .
$$

To conclude this section, let us mention that the heavy quark potential has also been investigated in a realization of the hard wall approximation: the used framework is the Randall-Sundrum model [45] which consists of an $A d S_{5}$ slice between two D3-branes with the fields of the Standard Model living on the four-dimensional world-volume of one of these branes [46]. Thermal effects have been studied by means of the Schwarzschild black hole-AdS metric [47]. The issue of finding general criteria for the confinement has also been considered in [48].

\section{The supergravity description of baryons}

\subsection{The baryon potential within the $A d S /$ CFT correspondence}

In a $S U(N)$ Yang-Mills theory, a colour-singlet baryon must be made of $N$ quarks. As described in the supergravity dual, such a baryon consists of $N$ quarks living on the boundary of a holographic space-time. On each of these quarks ends a string with the other endpoint attached to a D5-brane wrapped around the 5-sphere $S^{5}$ : the so-called baryon vertex located at the holographic coordinate $u_{0}[35,49]$. The typical radius of the baryon is denoted $r$. Moreover, the configuration of the $N$ quarks on the boundary is symmetric with respect to the boundary dimensions such that the resulting force acting on the baryon vertex is zero along these directions. In the following, we will consider only the induced metric contribution of the Dirac-Born-Infeld action of the D5-brane:

$$
S_{\mathrm{D} 5}=T_{5} \int d^{6} x \sqrt{\operatorname{det} g_{\mathrm{D} 5}}
$$


with $T_{5}^{-1}=(2 \pi)^{5} \alpha^{\prime 3} g_{s}$ the (inverse of the) tension of the brane ${ }^{3}$. The line element $d s_{\mathrm{D} 5}^{2}$ which measures invariant distances between two events located on the brane at $u_{0}$ can be derived straightforwardly from the $A d S_{5} \times S^{5}$ line element (3.57):

$$
d s_{\mathrm{D} 5}^{2}=\alpha^{\prime} \frac{u_{0}^{2}}{\tilde{R}^{2}} d t^{2}+\alpha^{\prime} \tilde{R}^{2} d \Omega_{5}^{2}=\alpha^{\prime} \frac{u_{0}^{2}}{\tilde{R}^{2}} d t^{2}+\alpha^{\prime} \tilde{R}^{2} g_{i j} d \theta^{i} d \theta^{j}=\alpha^{\prime} \frac{u_{0}^{2}}{\tilde{R}^{2}} d t^{2}+g_{i j} d \omega^{i} d \omega^{j}
$$

where we have defined the dimensionful coordinate $w^{i} \equiv\left(\sqrt{\alpha^{\prime}} \tilde{R}\right) \theta^{i}(i, j=1, \ldots, 5)$. Being the D5-brane static, the square root of the determinant of the induced metric in (6.207) does not depend on the time. The integral over the time coordinate $-T / 2 \geq t \geq T / 2$ gives rise to an overall factor $T$ in the action. The remaining integrals involves five coordinates describing the $S^{5}$. We have [50]:

$$
\begin{aligned}
S_{\mathrm{D} 5} & =\frac{1}{(2 \pi)^{5} \alpha^{\prime 3} g_{s}} \int_{-T / 2}^{T / 2} d t \int_{S^{5}} d^{5} x \sqrt{\alpha^{\prime} \frac{U_{0}^{2}}{\tilde{R}^{2}}} \sqrt{\operatorname{det} g_{S^{5}}}=\frac{T U_{0}}{(2 \pi)^{5}\left(\alpha^{\prime}\right)^{\frac{5}{2}} g_{s} \tilde{R}} \int_{S^{5}} d^{5} x \sqrt{\operatorname{det} g_{S^{5}}} \\
& =\frac{T U_{0}}{(2 \pi)^{5}\left(\alpha^{\prime}\right)^{\frac{5}{2}} g_{s} \tilde{R}}\left(\sqrt{\alpha^{\prime}} \tilde{R}\right)^{5} V\left(S^{5}\right)=\frac{T N U_{0}}{8 \pi}
\end{aligned}
$$

where $V\left(S^{5}\right)=\pi^{3}$ is the volume of the unit 5 -sphere and $\tilde{R}^{4}=4 \pi g_{s} N$. The NambuGoto action of a string world-sheet in the $A d S_{5} \times S^{5}$ background has already been widely studied (3.65)-(3.66). However, the baryonic system involves the additional contribution of the D5-brane. The total action is thus (with $\left.U(x=0) \equiv U_{0}\right)$ :

$$
S_{\text {total }}=S_{\mathrm{D} 5}+\sum_{i=1}^{N} S_{\text {string }}^{(i)}=\frac{T N U_{0}}{8 \pi}+\frac{T N}{2 \pi} \int_{0}^{r} d x \sqrt{U^{\prime 2}+\frac{U^{4}}{\tilde{R}^{4}}}
$$

Let us remark that the integral above over the boundary spatial coordinate $x$ runs from 0 to the typical radius $r$ of the baryon. This latter should not be confused with the inter-quark distance, also denoted $r$, in $Q \bar{Q}$ bound-states. (Besides, the dual string configurations associated to baryons and mesons are quite dissimilar.)

First of all, let us derive the stability condition of the baryon vertex along the holographic coordinate. Since the endpoint $U_{0}$ of the $N$ strings is free to vary $\delta U_{0} \neq 0$ and

\footnotetext{
${ }^{3}$ In general, a D $p$-brane carries on its $(p+1)$-dimensional world-volume electromagnetic fields of which the dynamics is governed by the so-called Dirac-Born-Infeld action:

$$
S_{\mathrm{Dp}}=T_{p} \int d^{p+1} x \sqrt{-\operatorname{det}\left(\eta_{M N}+2 \pi \alpha^{\prime} F_{M N}\right)}
$$

with $T_{p}=\frac{2 \pi}{\left(2 \pi \ell_{s}\right)^{p+1} g_{s}}$ the brane tension and $M, N=0,1, \ldots, p$ the space-time indices of the (flat) worldvolume of the $\mathrm{D} p$-brane.
} 
the variational principle gives:

$$
\begin{aligned}
\delta S_{\text {total }} & =\frac{N T}{2 \pi}\left\{\frac{\delta U_{0}}{4}+\int_{0}^{r} d x \frac{1}{\sqrt{U^{\prime 2}+\frac{U^{4}}{\tilde{R}^{4}}}}\left(\frac{2 U^{3}}{\tilde{R}^{4}} \delta U+U^{\prime} \frac{d}{d x} \delta U\right)\right\} \\
& =\frac{N T}{2 \pi}\left[\frac{\delta U_{0}}{4}+\int_{0}^{r} d x \frac{d}{d x}\left(\frac{U^{\prime} \delta U}{\sqrt{U^{\prime 2}+\frac{U^{4}}{\tilde{R}^{4}}}}\right)\right]+\frac{N T}{2 \pi} \int_{0}^{r} d x \delta U\left[\frac{2 U^{3}}{\tilde{R}^{4} \sqrt{U^{\prime 2}+\frac{U^{4}}{\tilde{R}^{4}}}}-\frac{d}{d x}\left(\frac{U^{\prime}}{\sqrt{U^{\prime 2}+\frac{U^{4}}{\tilde{R}^{4}}}}\right)\right]
\end{aligned}
$$

The first contribution in square brackets stands for the surface term at $U_{0}$ ( since $\delta U(r)=0$ on the boundary space) and must vanishes for any $\delta U_{0}$ which gives therefore the stability (or no-force) condition of the D5-brane along the holographic coordinate:

$$
\frac{U_{0}^{\prime}}{\sqrt{U_{0}^{\prime 2}+\frac{U_{0}^{4}}{\tilde{R}^{4}}}}=\frac{1}{4}
$$

where $\left.U_{0}^{\prime} \equiv \frac{d U}{d x}\right|_{x=0}$ is the slope of the $N$ strings at the baryon vertex. In particular, we have $U_{0}^{\prime 2}=\frac{1}{15} \frac{U_{0}^{4}}{\tilde{R}^{4}}$ which allow us to determine the value of the first integral (3.70) at $x=0$ in the baryon case:

$$
\frac{U^{4}}{\sqrt{U^{\prime 2}+\frac{U^{4}}{\tilde{R}^{4}}}}=\sqrt{\frac{15}{16}} \tilde{R}^{2} U_{0}^{2}=\text { const. }
$$

or, if we are interested in an expression of the derivative (always positive since $0 \leq x \leq r$ ):

$$
U^{\prime}(x)=\frac{U^{2}}{\tilde{R}^{2}} \sqrt{\beta^{2} \frac{U^{4}}{U_{0}^{4}}-1} .
$$

We have defined $\beta=\sqrt{\frac{16}{15}}$. The second contribution in square brackets must also vanish for any interior $\delta U$ and, thus, gives the equation of motion for $U(x)$.

The typical radius of the baryon has the expression:

$$
r\left(U_{0}\right)=\int_{0}^{r} d x=\int_{U_{0}}^{\infty} \frac{d U}{U^{\prime}} \underset{\left(v \equiv \frac{U}{U_{0}}\right)}{=} \frac{\tilde{R}^{2}}{U_{0}} \int_{1}^{\infty} \frac{d v}{v^{2} \sqrt{\beta^{2} v^{4}-1}}
$$

or

$$
U_{0}(r)=\frac{\tilde{R}^{2}}{r} \int_{1}^{\infty} \frac{d v}{v^{2} \sqrt{\beta^{2} v^{4}-1}} .
$$

The contribution of one string $(i)$ to the energy of the baryon is obtained in a way similar 
to $(3.83)$ :

$$
\begin{aligned}
V_{\text {string }}^{(i)}\left(U_{0}\right) & =\lim _{T \rightarrow \infty} \frac{1}{T} S_{\text {string }}^{(i)}=\frac{1}{2 \pi} \int_{0}^{r} d x \sqrt{U^{2}+\frac{U^{4}}{\tilde{R}^{4}}} \\
& =\frac{1}{2 \pi} \int_{U_{0}}^{\infty} d U \beta \frac{U^{2}}{U_{0}^{2}}\left(\beta^{2} \frac{U^{4}}{U_{0}^{4}}-1\right)^{-\frac{1}{2}} \\
V_{\text {string }}^{(\text {reg. })(i)}\left(U_{0}, U_{\max }\right) \underset{\left(v \equiv \frac{U}{U_{0}}\right)}{=} & \frac{U_{0}}{2 \pi} \int_{1}^{U_{\max } / U_{0}} d v\left[\frac{\beta v^{2}}{\sqrt{\beta^{2} v^{4}-1}}-1\right]+\frac{U_{0}}{2 \pi} \int_{1}^{U_{\max } / U_{0}} d v \\
V_{\text {string }}^{(R)(i)}\left(U_{0}\right) & =\frac{U_{0}}{2 \pi}\left\{\int_{1}^{\infty} d v\left[\frac{\beta v^{2}}{\sqrt{\beta^{2} v^{4}-1}}-1\right]-1\right\} .
\end{aligned}
$$

So, the energy of the baryon is:

$$
V_{B}^{(R)}\left(U_{0}\right)=\frac{N U_{0}}{8 \pi}+\frac{N U_{0}}{2 \pi}\left\{\int_{1}^{\infty} d v\left[\frac{\beta v^{2}}{\sqrt{\beta^{2} v^{4}-1}}-1\right]-1\right\} .
$$

In terms of the typical radius $r$, we obtain a potential which is proportional to $N$ times the potential of a quark-antiquark bound-state (3.89) ( $\lambda$ is the 't Hooft coupling constant):

$$
V_{B}(r)=-N \alpha_{B} \frac{\sqrt{2 \lambda}}{r}
$$

with

$$
\alpha_{B}=\frac{1}{2 \pi} \int_{1}^{\infty} \frac{d u}{u^{2} \sqrt{\beta^{2} u^{4}-1}}\left\{\frac{3}{4}-\int_{1}^{\infty} d v\left[\frac{\beta v^{2}}{\sqrt{\beta^{2} v^{4}-1}}-1\right]\right\} \simeq 0.036 .
$$

The behaviour in $1 / r$ of the baryon potential is obviously dictated by the conformal invariance of the field theory at the boundary.

\subsection{Existence of $A d S / C F T$ baryons made of $k<N$ quarks}

Remarkably, another string configuration has been identified which allows, on the supergravity side, to account for baryons made of a smaller number of quark constituents $k<N$ [50]. In that case, to the baryon vertex at $u_{0}$ are attached $k$ strings, quite analogous to those studied above, which end, at the boundary $(u \rightarrow+\infty)$, on the $k$ quarks. However, there are $N-k$ remaining strings which stretch out from the baryon vertex to the brane at $u=0$. These strings are radial straight strings and are described by the action $(j=k+1, \ldots, N-k)$ :

$$
S_{\text {string }}^{(j)}=\frac{1}{2 \pi \alpha^{\prime}} \int d^{2} \xi \sqrt{\operatorname{det}\left(\gamma_{a b}\right)}=\frac{1}{2 \pi \alpha^{\prime}} \int_{-T / 2}^{T / 2} d t \int_{0}^{U_{0}} d U \sqrt{\alpha^{\prime 2}}=\frac{T U_{0}}{2 \pi}
$$


since the non-vanishing components of the induced metric tensor on the world-sheet are here $\left(\xi^{1}=U\right.$ and $\left.\xi^{2}=t\right)$ :

$$
\begin{aligned}
& \gamma_{11}=\alpha^{\prime} \frac{\tilde{R}^{2}}{U^{2}}, \\
& \gamma_{22}=\alpha^{\prime} \frac{U^{2}}{\tilde{R}^{2}} .
\end{aligned}
$$

Hence, the total action governing the dynamics of the baryon:

$$
\begin{aligned}
S_{\text {total }} & =S_{\mathrm{D} 5}+\sum_{i=1}^{k} S_{\text {string }}^{(i)}+\sum_{j=1}^{N-k} S_{\text {string }}^{(j)} \\
& =\frac{T N U_{0}}{8 \pi}+\frac{k T}{2 \pi} \int_{0}^{r} d x \sqrt{U^{\prime 2}+\frac{U^{4}}{\tilde{R}^{4}}}+\frac{T(N-k) U_{0}}{2 \pi} .
\end{aligned}
$$

The variational principle gives then the following stability condition for the baryon vertex along the holographic coordinate:

$$
\left.\delta S_{\text {total }}\right|_{\substack{\text { surface } \\ \text { term at } U_{0}}}=0 \Rightarrow \frac{U_{0}^{\prime}}{\sqrt{\frac{U_{0}^{4}}{\tilde{R}^{4}}+U_{0}^{\prime 2}}}=\frac{5 N-4 k}{4 k} \equiv A \Rightarrow U_{0}^{\prime 2}=\frac{A^{2}}{1-A^{2}} \frac{U_{0}^{4}}{\tilde{R}^{4}} .
$$

If $k=N$, then $A=\frac{1}{4}$ and we recover (6.213). If the baryon has less quarks $k \leq N$ then $A \geq \frac{1}{4}$. On the other hand, the upper bound for $A$ (which corresponds to the lower bound for $k$ ) is obtained for radial straight $k$-type strings ending on the baryon vertex such that $U_{0}^{\prime} \rightarrow \infty$. Then, $A=1$ and $k=\frac{5 N}{8}$. To summarize, the condition for having a stable string/brane system into the bulk demands $\frac{5 N}{8} \leq k \leq N$.

The Lagrangian density in (6.225) depends on $x$ only through $U(x)$. It results the first integral:

$$
\frac{U^{4}}{\sqrt{U^{\prime 2}+\frac{U^{4}}{\tilde{R}^{4}}}}=\sqrt{1-A^{2}} \tilde{R}^{2} U_{0}^{2}
$$

which can be put into the form:

$$
U^{\prime}(x)=\frac{U^{2}}{\tilde{R}^{2} \sqrt{1-A^{2}}} \sqrt{\frac{U^{4}}{U_{0}^{4}}-\left(1-A^{2}\right)} .
$$

The radius and the potential of these "reduced" baryons are then:

$$
r\left(U_{0}\right)=\frac{\tilde{R}^{2}}{U_{0}} \sqrt{1-A^{2}} \int_{1}^{\infty} \frac{d v}{v^{2} \sqrt{v^{4}-\left(1-A^{2}\right)}}
$$

and

$$
\begin{aligned}
V_{B}\left(U_{0}\right) & =\frac{N U_{0}}{8 \pi}+\frac{(N-k) U_{0}}{2 \pi}+\frac{k}{2 \pi} \int_{0}^{r} d x \sqrt{U^{2}+\frac{U^{4}}{\tilde{R}^{4}}} \\
& =\frac{N U_{0}}{8 \pi}+\frac{(N-k) U_{0}}{2 \pi}+\frac{k U_{0}}{2 \pi} \int_{1}^{\infty} d v \frac{v^{2}}{\sqrt{v^{4}-\left(1-A^{2}\right)}}
\end{aligned}
$$




$$
\begin{gathered}
V_{B}^{(\text {reg. })}\left(U_{0}, U_{\text {max }}\right)=\frac{N U_{0}}{8 \pi}+\frac{(N-k) U_{0}}{2 \pi}+\frac{k U_{0}}{2 \pi} \int_{1}^{U_{\max } / U_{0}} d v\left[\frac{v^{2}}{\sqrt{v^{4}-\left(1-A^{2}\right)}}-1\right]+\frac{k}{2 \pi}\left(U_{\max }-U_{0}\right) \\
V_{B}^{(R)}\left(U_{0}\right)=\frac{N U_{0}}{8 \pi}+\frac{(N-k) U_{0}}{2 \pi}+\frac{k U_{0}}{2 \pi}\left\{\int_{1}^{\infty} d v\left[\frac{v^{2}}{\sqrt{v^{4}-\left(1-A^{2}\right)}}-1\right]-1\right\}
\end{gathered}
$$

where the counter-term required in order to absorb the UV singularity consists here of $k$ radial straight strings stretched out from the boundary at $u_{\max }$ to the brane at $u=0$ (the contribution of the baryon vertex vanishes according to $(6.210)$ with $U_{0}=0$ ):

$$
V_{c . t .}=-\lim _{U_{\max } \rightarrow \infty} k \frac{U_{\max }}{2 \pi}
$$

When $k=N$, (6.227)-(6.230) reduces to (6.214)-(6.216) and (6.219) respectively. If $k=\frac{5 N}{8}$ then $A=1$ which implies $r\left(U_{0}\right)=0$ (the baryon size vanishes) and $V_{B}^{(R)}\left(U_{0}\right)=0$ independently of the location $U_{0}$ of the D5-brane along the holographic coordinate. If $\frac{5 N}{8}<k \leq N$ then $A<1$ and the baryon energy $V_{B}^{(R)}(r)=-\alpha U_{0}(r)$ can be written as the product of a negative constant $-\alpha(\alpha>0)$ with $U_{0}$ (expressed in terms of $r$ ) [50].

\subsection{Baryons in three-dimensional Yang-Mills theory}

As largely discussed in preceding sections, we consider a spatial string/brane configuration in the Schwarzschild black hole- $A d S_{5}$ background (4.90). The Nambu-Goto action of one space-like string world-sheet reads $(i=1, \ldots, N)$

$$
S_{\text {string }}^{(i)}=\frac{Y}{2 \pi} \int_{0}^{r} d x \sqrt{\frac{U^{4}}{\tilde{R}^{4}}+\frac{U^{4}}{U^{4}-U_{T}^{4}} U^{\prime 2}}
$$

which is obviously reminiscent of (4.113), and the total action is:

$$
S_{\text {total }}=S_{\mathrm{D} 5}+\sum_{i=1}^{N} S_{\text {string }}^{(i)}=\frac{Y N U_{0}}{8 \pi}+\frac{N Y}{2 \pi} \int_{0}^{r} d x \sqrt{\frac{U^{4}}{\tilde{R}^{4}}+\frac{U^{4}}{U^{4}-U_{T}^{4}} U^{2}} .
$$

Apart from the equation of motion for the string coordinate $U(x)$, the variational principle gives the following surface term at $U_{0}$ where the baryon vertex wraps the 5 -sphere $S^{5}$ $\left(\delta U_{0} \neq 0\right.$ while $\left.\delta U(r)=0\right)$ :

$$
\left.\delta S_{\text {total }}\right|_{\text {term at } U_{0}} ^{\text {surface }}=0 \Rightarrow \frac{U_{0}^{\prime}}{\left(1-\frac{U_{T}^{4}}{U_{0}^{4}}\right) \sqrt{\frac{U_{0}^{4}}{\tilde{R}^{4}}+\frac{U_{0}^{\prime 2}}{1-\frac{U_{T}^{4}}{U_{0}^{4}}}}}=\frac{1}{4} .
$$

At zero temperature where $u_{T}=\pi \tilde{R}^{2} T=0$, we recover the stability condition (6.213).

We are interested in the large distance regime where the typical radius of the YangMills baryons is large. That corresponds to the situation where the D5-brane reaches the 
horizon $\left(U_{0} \rightarrow U_{T}\right)$. Then, according to (6.234), the slope of the strings must vanish at the horizon $\left(U_{0}^{\prime} \rightarrow 0\right)$ : the $N$ strings, attached to the quarks on the boundary, become radial straight strings but, contrary to the $A d S / C F T$ baryons made of $k=\frac{5 N}{8}$ quarks considered previously, the radius of the Yang-Mills baryons remains sizeable. Indeed, once they hit the event horizon at $u_{T}$, the strings spread along the transverse directions to the holographic dimension up to the baryon vertex. In this limit case, the first integral derived from (6.233) reduced to (4.114):

$$
\frac{U^{4}}{\sqrt{\frac{U^{4}}{\tilde{R}^{4}}+\frac{U^{4}}{U^{4}-U_{T}^{4}} U^{\prime 2}}}=\frac{U_{0}^{4}}{\sqrt{\frac{U_{0}^{4}}{\tilde{R}^{4}}+\frac{U_{0}^{4}}{U_{0}^{4}-U_{T}^{4}} U_{0}^{\prime 2}}} \underset{\left(U_{0}^{\prime} \rightarrow 0\right)}{\simeq} \tilde{R}^{2} U_{0}^{2}=\text { const. }
$$

It sheds light to express the radius (denoted $r_{B}$ here in order to distinguish it from the meson inter-quark distance) and the energy of the baryons in terms of the quark separation (4.116) and of the potential (4.122) of the $Q \bar{Q}$ bound-states in three-dimensional YangMills theory. We have at large distances $\left(U_{0} \simeq U_{T}\right)$ :

$$
\begin{aligned}
r_{B}\left(U_{0}, U_{T}\right) & \simeq \frac{1}{2} r\left(U_{0}, U_{T}\right) \\
V_{B}^{(R)}\left(U_{0}, U_{T}\right) & \simeq \frac{N U_{T}}{8 \pi}+\frac{N}{2} V_{Q \bar{Q}}^{(R)}\left(U_{0}, U_{T}\right) .
\end{aligned}
$$

The integrals in $r_{B}\left(U_{0}, U_{T}\right)$ and $V_{B}^{(R)}\left(U_{0}, U_{T}\right)$ diverge which gives rise, by identifying their singular contributions, to a confining linear potential with a string tension equals to $N$ times the mesonic string tension (4.146):

$$
V_{B}^{(R)}(r)=N\left(\frac{1}{2} \pi \tilde{R}^{2} T^{2}\right) r
$$

\section{Conclusion}

The expectation value of the Wilson loop $W[\mathcal{C}]$ provides, through the area law at large distances, a criterion for the confinement. According to the $A d S / C F T$ prescription [4], it can also be evaluated, on the supergravity side, from the classical Nambu-Goto action of a string world-sheet lying on the closed loop $\mathcal{C}$ at the boundary. Since the world-sheet is no longer forced to span only the four-dimensional boundary space-time but can spread out along the fifth holographic coordinate, we do not expect to necessarily recover the area law of the Wilson loop (which is indeed a four-dimensional space-time result). In the absence of any length scale, the interaction potentials of hadrons exhibit a (non-perturbative) Coulomb-like behaviour $V(r) \propto-1 / r$ in agreement with the underlying conformal invariance of the boundary theory. On the contrary, provided that a dimensionful parameter is introduced in the formalism (which can be the Beckenstein-Hawking temperature or 
by means of a warp factor in the $A d S_{5}$ metric), the linear confinement $V(r) \propto r$ arises corresponding to the situation where string world-sheet reaches a stationary point along the holographic coordinate for which the inter-quark distance $r$ explodes.

\section{Acknowledgement}

Most of the material exposed herehas been compiled during my INFN fellowship in Bari, Italy while I was working on the phenomenology of the IR Soft Wall Model. Especially, I am grateful to P. Colangelo for having made my stay there so fruitful and exciting. This work was partially supported by the Theoretical Physics Center for Science Facilities (TPCSF), Institute of High Energy Physics (IHEP), Chinese Academy of Sciences (CAS).

\section{A Brief review of the Wilson loop in QCD}

When one attempts to formulate QCD in a discretized space-time, one is naturally led to introduce the so-called Wilson loop $W[\mathcal{C}]$ (especially, when one tries to build a gauge invariant action for the gluon fields) of which the large distance behaviour provides a confinement criterion [25]. The (Euclidean version of the) area law:

$$
W[\mathcal{C}]=e^{-\sigma_{t} r T}
$$

where the contour $\mathcal{C}$ is taken as a rectangle with time-like and space-like sides of length $T$ and $r$ respectively, is equivalent to a confining interaction potential ( $r$ is the inter-quark distance):

$$
V(r)=\sigma_{t} r
$$

The Wilson loop is consequently a key ingredient of lattice QCD and plays a fundamental role for the study of non-perturbative properties of QCD.

We might wonder whether the Wilson loop plays such an important role in the continuous theory. As a matter of fact, it turns out to be at the basis of a formulation of QCD where all the references to the gauge invariance of the theory (gauge transformations, gauge-fixing terms, etc...) are discarded. Within this framework, QCD equations become functional equations of the Wilson loop (or rather, of its multi-loop generalizations): their resolving would then bring us valuable information on the behaviour of QCD. 


\section{A.1 The gauge line}

QCD is a non-Abelian gauge theory: the Lagrangian density $\mathcal{L}_{Q C D}$ is invariant under local transformations of the quark field phases, which requires the presence of self-interacting gluon fields in the theory. Being local, the transformations involve space-time dependent parameters. That explains why the Dirac mass term $-m \bar{q}(x) q(x)$ is allowed in $\mathcal{L}_{Q C D}$ whereas the non-gauge invariant bilocal term $\bar{q}(y) q(x)$ is not for instance. So, which sense to give to the partial derivative of the quark field $\partial_{\mu} q(x)$ in a direction $\hat{\mu}$ :

$$
\partial_{\mu} q(x) \equiv \lim _{\epsilon \rightarrow 0} \frac{q(x+\epsilon \hat{\mu})-q(x)}{\epsilon}
$$

which indeed involves two different space-time events. The gauge transformation of $\partial_{\mu} q(x)$ seems intricate since to each point $x$ and $x+\epsilon \hat{\mu}$ corresponds a different transformation law. To resolve this issue, one introduces a non-local object, namely a phase factor or gauge line $U$ such that $U(x+\epsilon \hat{\mu}) q(x)$ and $q(x+\epsilon \hat{\mu})$ satisfy the same transformation law. In other words, $U(x+\epsilon \hat{\mu})$ brings the gauge transformation from the point $x$ to the point $x+\epsilon \hat{\mu}$. The new derivative is then defined as:

$$
D_{\mu} q(x) \equiv \lim _{\epsilon \rightarrow 0} \frac{q(x+\epsilon \hat{\mu})-U(x+\epsilon \hat{\mu}) q(x)}{\epsilon}
$$

which is nothing else than the standard covariant derivative:

$$
D_{\mu} q(x)=\left(\partial_{\mu}+i g A_{\mu}(x)\right) q(x)
$$

such that

$$
\begin{aligned}
q(x) & \rightarrow q^{\prime}(x)=\Omega(x) q(x), \\
D_{\mu} q(x) & \rightarrow D^{\prime}{ }_{\mu} q^{\prime}(x)=\Omega(x) D_{\mu} q(x) .
\end{aligned}
$$

$A_{\mu}(x)$ are the gauge fields, $\Omega(x)=e^{i g \omega(x)}$ is an element of the gauge group $S U\left(N_{c}\right)$ and $g$ is the strong coupling constant. In this appendix, the number of colours $N_{c}$ is regarded as a free parameter allowed to take all the possible positive integers. As a result, we have $N_{c}^{2}-1$ (the dimension of the group) real gauge parameters $\omega^{i}(x)$ : the hermitian parameter matrix reads $\omega(x) \equiv \omega^{i}(x) \frac{\lambda^{i}}{2}$ and the $\frac{\lambda^{i}}{2}$ 's are the infinitesimal generators of the algebra $s u\left(N_{c}\right)\left(i=1,2, \ldots, N_{c}^{2}-1\right)$.

The definition of the gauge line is the following:

$$
U(y, x ; \mathcal{C}) \equiv P e^{-i g \int_{x}^{y} A_{\mu}(x) d x^{\mu}}
$$

which consists of a line integral of the gauge fields $A_{\mu}(x)$ : thus, $U(y, x ; \mathcal{C})$ depends on the path $\mathcal{C}$ oriented from $x$ to $y$. In the differential geometry framework, one says that $U(y, x ; \mathcal{C})$ performs a parallel transport from $x$ to $y$ and that $A_{\mu}(x)$ is the corresponding connection. The prescription $P$ in $(1.245)$ is the path ordering operator, required in 
order to take into account the non-Abelian nature of QCD. Indeed, the gauge fields $A_{\mu}(x) \equiv A_{\mu}^{i}(x) \frac{\lambda^{i}}{2}$ are anti-commuting square matrices of order $N_{c}$.

A path $\mathcal{C}(\sigma)$ (so, a mapping function) can be parametrized as follows:

$$
\begin{gathered}
\mathcal{C}: \quad[0,1] \rightarrow \mathcal{M}^{4} \\
\sigma \rightarrow x^{\mu}(\sigma)
\end{gathered}
$$

where $\mathcal{M}^{4}$ is the $(3+1)$-dimensional Minkowski space-time with the flat metric tensor $\eta_{\mu \nu}=\operatorname{diag}(+1,-1,-1,-1)$ and the parameter $\sigma \in[0,1]$ such that $x^{\mu}(\sigma=0) \equiv x^{\mu}$ and $x^{\mu}(\sigma=1) \equiv y^{\mu}$. Moreover, $d x^{\mu}=\dot{x}^{\mu}(\sigma) d \sigma$ where $\dot{x}^{\mu}=\frac{d x^{\mu}}{d \sigma}$ is the $\mu^{\text {th }}$ component of the four-vector tangent to $\mathcal{C}$ at $x(\sigma)$. The gauge line (1.245) has then the following parametric representation:

$$
U(y, x ; \mathcal{C})=P e^{-i g \int_{0}^{1} d \sigma \dot{x}^{\mu}(\sigma) A_{\mu}(x(\sigma))}
$$

and is invariant:

- by reparametrization: $x(\sigma) \rightarrow x\left(\sigma^{\prime}\right)$ where $\sigma^{\prime}=f(\sigma)$ with $f^{\prime}(\sigma)>0$ in order to keep unchanged the point ordering along $\mathcal{C}$.

- under transformations of Poincaré's group (the flat space-time isometry group): $x^{\mu}(\sigma) \rightarrow x^{\prime \mu}(\sigma)=\Lambda^{\mu}{ }_{\nu} x^{\nu}(\sigma)+a^{\mu}$ where the Lorentz matrix $\Lambda^{\mu}{ }_{\nu}$ and the translation parameter $a^{\mu}$ are constant.

One sees that to each generator $\frac{\lambda^{i}}{2}$ is associated, via the gauge field $A_{\mu}^{i}(x(\sigma))$, one and only one value of $\sigma$ : the operator $P$ puts in order, through the parameter $\sigma$, the $s u\left(N_{C}\right)$ generators $\frac{\lambda^{i}}{2}$ 's along $\mathcal{C}$. Let us now divide the path $\mathcal{C}$ into infinitesimal straight lines $\delta \mathcal{C}$ 's, it is then possible to express the phase factor $U(y, x ; \mathcal{C})$ as an infinite product of elementary gauge lines, each of them being associated with its own contour $\delta \mathcal{C}$ and ordered along $\mathcal{C}$ according to the prescription $P$ :

$$
U(y, x ; \mathcal{C})=\lim _{n \rightarrow \infty} \prod_{n} U\left(x_{n}, x_{n-1} ; \delta \mathcal{C}\right)
$$

Another expression for $U(y, x ; \mathcal{C})$ makes use of the parametric representation $(1.247)$ :

$$
\begin{aligned}
U_{b}^{a}(y, x ; \mathcal{C})= & \sum_{n=0}^{\infty}\left(-\frac{i g}{\sqrt{2}}\right)^{n} \int_{0}^{1} d \sigma_{1} \ldots \int_{0}^{1} d \sigma_{n} \theta\left(\sigma_{1}-\sigma_{2}\right) \ldots \theta\left(\sigma_{n-1}-\sigma_{n}\right) \\
& \times \dot{x}^{\mu_{1}}\left(\sigma_{1}\right) \ldots \dot{x}^{\mu_{n}}\left(\sigma_{n}\right) A_{\mu_{1}{ }_{c_{1}}^{a}}\left(\sigma_{1}\right) \ldots A_{\mu_{n} b}^{c_{n-1}}\left(\sigma_{n}\right)
\end{aligned}
$$

where we have written $A_{\mu}(x(\sigma)) \equiv A_{\mu}(\sigma)$ for the sake of simplicity and defined the matrix element $\left(A_{\mu}\right)_{b}^{a}=\left(A_{\mu}^{i} \frac{\lambda^{i}}{2}\right)_{b}^{a} \equiv \frac{1}{\sqrt{2}} A_{\mu_{b}}^{a}\left(a, b=1,2, \ldots, N_{c}\right)$. At each order in the expansion, the operator $P$ puts in order the gauge field product from the right to the left by increasing value of $\sigma$. The Heaviside functions play the role of the prescription $P$ and compensate for the factors of $1 / n$ ! usually present when expanding the exponential function. (The 
expansion (1.249) is similar to the well-known QFT expansion of the evolution operator in interaction representation.)

Because the gauge line $U(y, x ; \mathcal{C})$ carries out a parallel transport from $x$ to $y$ along the path $\mathcal{C}(\sigma)$, the fields $q(y)$ and $U(y, x ; \mathcal{C}) q(x)$ satisfy the same transformation law (thus, the operator $\bar{q}(y) U(y, x ; \mathcal{C}) q(x)$ is manifestly gauge invariant) which implies the following gauge transformation for $U(y, x ; \mathcal{C})$ :

$$
U(y, x ; \mathcal{C}) \rightarrow U^{\prime}(y, x ; \mathcal{C})=\Omega(y) U(y, x ; \mathcal{C}) \Omega^{\dagger}(x)
$$

Then, the corresponding matrix element $U_{b}^{a}(y, x ; \mathcal{C})$ transforms as follows:

$$
U_{b}^{a}(y, x ; \mathcal{C}) \rightarrow U_{b}^{\prime a}(y, x ; \mathcal{C})=\Omega_{c}^{a}(y) U_{d}^{c}(y, x ; \mathcal{C}) \Omega_{b}^{d}(x)^{\dagger}
$$

from which can be easily obtained the infinitesimal gauge transformation:

$$
\delta_{G} U_{b}^{a}(y, x ; \mathcal{C})=i g\left[w_{c}^{a}(y) U_{b}^{c}(y, x ; \mathcal{C})-U_{c}^{a}(y, x ; \mathcal{C}) w_{b}^{c}(x)\right]
$$

\section{A.2 Mandelstam's formula}

Mandelstam's formula [51] is one of the most important equation when one attempts to rewrite Yang-Mills theory within the loop space formalism (defined as the set of all the continuous closed curves):

$$
\frac{\delta}{\delta \sigma^{\mu \nu}(z)} U(y, x ; \mathcal{C})=-i g U(y, z ; \mathcal{C}) G_{\mu \nu}(z) U(z, x ; \mathcal{C})
$$

with $G_{\mu \nu}(z)=\partial_{\mu} A_{\nu}(z)-\partial_{\nu} A_{\mu}(z)+i g\left[A_{\mu}, A_{\nu}\right](z)$ the non-Abelian strength field tensor. This relation can be derived as follows. Thanks to the path ordering prescription $P$, the gauge line (1.245) can be written as the product of three phase factors:

$$
U(y, x ; \mathcal{C})=U\left(y, x_{2} ; \mathcal{C}\right) U\left(x_{2}, x_{1} ; \mathcal{C}\right) U\left(x_{1}, x ; \mathcal{C}\right)
$$

where each phase factor corresponds to one of the three stretches $\left(x, x_{1}\right),\left(x_{1}, x_{2}\right)$ and $\left(x_{2}, y\right)$ of $\mathcal{C}$. The two points $x_{1}$ and $x_{2}$ are located anywhere between the endpoints $x$ and $y$ of the curve. Let us then perform an infinitesimal variation $\delta \mathcal{C}$ of the section $\left(x_{1}, x_{2}\right)$ :

$$
\left\{\begin{aligned}
\mathcal{C} & \rightarrow \mathcal{C}^{\prime}=\mathcal{C}+\delta \mathcal{C} \\
U\left(x_{2}, x_{1} ; \mathcal{C}\right) & \rightarrow U\left(x_{2}, x_{1} ; \mathcal{C}^{\prime}\right)
\end{aligned}\right.
$$

The gauge line corresponding to the new curve $\mathcal{C}^{\prime}$ reads:

$$
U\left(y, x ; \mathcal{C}^{\prime}\right)=U\left(y, x_{2} ; \mathcal{C}\right) U\left(x_{2}, x_{1} ; \mathcal{C}+\delta \mathcal{C}\right) U\left(x_{1}, x ; \mathcal{C}\right)
$$


One defines the variation of the gauge line when passing from the curves $\mathcal{C}$ to $\mathcal{C}^{\prime}$ by the difference:

$$
\begin{aligned}
\delta_{\mathcal{C}} U(y, x ; \mathcal{C}) & =U\left(y, x ; \mathcal{C}^{\prime}\right)-U(y, x ; \mathcal{C}) \\
& =U\left(y, x_{2} ; \mathcal{C}\right)\left[U\left(x_{2}, x_{1} ; \mathcal{C}+\delta \mathcal{C}\right)-U\left(x_{2}, x_{1} ; \mathcal{C}\right)\right] U\left(x_{1}, x ; \mathcal{C}\right) \\
\delta_{\mathcal{C}} U(y, x ; \mathcal{C}) & =U\left(y, x_{2} ; \mathcal{C}\right)\left[U\left(x_{2}, x_{1} ; \mathcal{C}+\delta \mathcal{C}\right) U^{-1}\left(x_{2}, x_{1} ; \mathcal{C}\right)-1\right] U\left(x_{2}, x_{1} ; \mathcal{C}\right) U\left(x_{1}, x ; \mathcal{C}\right)
\end{aligned}
$$

The phase factor $U^{-1}\left(x_{2}, x_{1} ; \mathcal{C}\right)$ corresponds to the section $\left(x_{1}, x_{2}\right)$ of the contour $\mathcal{C}$ but oriented from $x_{2}$ to $x_{1}: U^{-1}\left(x_{2}, x_{1} ; \mathcal{C}\right)=U\left(x_{1}, x_{2} ; \mathcal{C}\right)$. Consequently, the product $U\left(x_{2}, x_{1} ; \mathcal{C}+\delta \mathcal{C}\right) U^{-1}\left(x_{2}, x_{1} ; \mathcal{C}\right)$ in $(1.257)$ stands for a closed curve or loop, denoted $C$. If the modification $\delta \mathcal{C}$ of the contour $\mathcal{C}$ is infinitesimal, this loop $C$ gives rise to a surface of infinitesimal area $\delta S$. We can then apply the non-Abelian Stockes theorem valid for infinitesimal loops:

$$
e^{\oint_{C} A_{\mu} d x^{\mu}}=e^{\iint_{\delta S} d \sigma_{\mu \nu}(z) G^{\mu \nu}(z)}
$$

such that

$$
\begin{aligned}
U\left(x_{2}, x_{1} ; \mathcal{C}+\delta \mathcal{C}\right) U^{-1}\left(x_{2}, x_{1} ; \mathcal{C}\right) & =P e^{-i g \int_{\mathcal{C}+\delta \mathcal{C}} A_{\mu} d x^{\mu}} P e^{i g \int_{\mathcal{C}} A_{\mu} d x^{\mu}} \\
& =P e^{-i g \oint_{C} A_{\mu} d x^{\mu}} \\
U\left(x_{2}, x_{1} ; \mathcal{C}+\delta \mathcal{C}\right) U^{-1}\left(x_{2}, x_{1} ; \mathcal{C}\right) & =P e^{-i g \iint_{\delta S} d \sigma_{\mu \nu}(z) G^{\mu \nu}(z)}
\end{aligned}
$$

where $d \sigma_{\mu \nu}(z)=d z_{\mu} \wedge d z_{\nu}$ is the area element $\left(d \sigma_{\mu \nu}=-d \sigma_{\nu \mu}\right)$ with the internal point $z$ located between $x_{1}$ and $x_{2}$ along $\mathcal{C}$.

We see that $U\left(x_{2}, x_{1} ; \mathcal{C}+\delta \mathcal{C}\right) U^{-1}\left(x_{2}, x_{1} ; \mathcal{C}\right)$ performs in $(1.257)$ a rotation of the gauge field $A_{\mu}$ along the loop $C$. According to the non-Abelian Stockes theorem (1.258), this rotation is related to the flux (1.259) of the strength field tensor $G_{\mu \nu}$ through a surface with the loop $C$ as boundary (in the differential geometry formalism, $G_{\mu \nu}$ is the curvature tensor of the internal colour space). Then, Mandelstam's formula can be obtained by expanding (1.257) at the leading order in $\delta S$ :

$\delta_{\mathcal{C}} U(y, x ; \mathcal{C})=U\left(y, x_{2} ; \mathcal{C}\right)\left[1-i g \iint_{\delta S} d \sigma_{\mu \nu}(z) G^{\mu \nu}(z)-1\right] U\left(x_{2}, x_{1} ; \mathcal{C}\right) U\left(x_{1}, x ; \mathcal{C}\right)+O\left(\delta S^{2}\right)$

so that

$$
\frac{\delta}{\delta \sigma^{\mu \nu}(z)} U(y, x ; \mathcal{C})=-i g U(y, z ; \mathcal{C}) G_{\mu \nu}(z) U(z, x ; \mathcal{C})
$$

by deriving with respect to the area element at any point $z$ between $x_{1}$ and $x_{2}$. In brief, the area derivative $\delta / \delta \sigma^{\mu \nu}(z)$ consists in inserting, at the point $z$ along the contour $\mathcal{C}$, the non-Abelian strength field tensor $G_{\mu \nu}(z)$. 
Although elegant, this derivation presents a certain number of shortcomings: first of all, the demonstration is geometrical and depends on the form of the curves $\mathcal{C}$ and $\mathcal{C}^{\prime}$. Secondly, the functional derivation (1.261) is not mathematically well-defined because arbitrary. Indeed, there are several possible definitions $[52,53]$ such as:

$$
\frac{\delta}{\delta \sigma_{\mu \nu}(z)} U(y, x ; \mathcal{C}) \equiv \lim _{\left|\delta \sigma_{\mu \nu}\right| \rightarrow 0} \frac{1}{\left|\delta \sigma_{\mu \nu}\right|}\left(U\left(y, x ; \mathcal{C}+\delta \mathcal{C}_{\mu \nu}(z)\right)-U(y, x ; \mathcal{C})\right)
$$

as used by Mandelstam where $\delta \mathcal{C}_{\mu \nu}(z)$ is the infinitesimal loop oriented along the plane $(\mu, \nu)$ at the point $z$ and $\left|\delta \sigma_{\mu \nu}\right|$ is the area of the associated minimal surface. In order to avoid these difficulties, it is therefore interesting to find another way to derive Mandelstam's formula (1.261). The solution consists in working with the (geometry independent) parametric representation of the gauge line (1.247). Let us write the variation of $U(y, x ; \mathcal{C})$ under an arbitrary transformation of $\mathcal{C}$ following the notations $U\left(x(\sigma), x\left(\sigma^{\prime}\right)\right) \equiv U\left(\sigma, \sigma^{\prime}\right)$, $A_{\alpha}(x(\sigma)) \equiv A_{\alpha}(\sigma)$ and $G_{\beta \alpha}(x(\sigma)) \equiv G_{\beta \alpha}(\sigma)[54]$ :

$$
\begin{aligned}
\delta_{\mathcal{C}} U(y, x ; \mathcal{C})= & -i g \delta x^{\alpha}(1) A_{\alpha}(1) U(1,0)+i g U(1,0) A_{\alpha}(0) \delta x^{\alpha}(0) \\
& +i g \int_{0}^{1} d \sigma U(1, \sigma) \dot{x}^{\beta}(\sigma) G_{\beta \alpha}(\sigma) \delta x^{\alpha}(\sigma) U(\sigma, 0) .
\end{aligned}
$$

The first two terms on the r.h.s. of (1.263) correspond to the variations of the endpoints $y \equiv x(\sigma=1)$ and $x \equiv x(\sigma=0)$ respectively. The last term involves all the points $x(\sigma)$ of $\mathcal{C}$ and thus corresponds to the internal contribution associated with the global deformation of $\mathcal{C}$. Let us perform the functional derivation with respect to an internal point $x^{\alpha}(\sigma)$ along the contour $\mathcal{C}(0<\sigma<1$ such that only the last term in (1.263) contributes):

$$
\frac{\delta U(y, x ; \mathcal{C})}{\delta x^{\alpha}(\sigma)}=i g U(1, \sigma) \dot{x}^{\beta}(\sigma) G_{\beta \alpha}(\sigma) U(\sigma, 0)
$$

A partial derivative with respect to $\dot{x}^{\beta}(\sigma)$ gives then Mandelstam's formula:

$$
\frac{\partial}{\partial \dot{x}^{\beta}(\sigma)} \frac{\delta U(y, x ; \mathcal{C})}{\delta x^{\alpha}(\sigma)}=-i g U(1, \sigma) G_{\alpha \beta}(\sigma) U(\sigma, 0)
$$

where $x(\sigma)$ and $\dot{x}(\sigma)$ have to be considered as independent operators. Comparing (1.261) with $(1.265)$, we obtain the following relations between the surface and functional derivatives with respect to the point $x(\sigma)$ :

$$
\begin{aligned}
\frac{\partial}{\partial \dot{x}^{\beta}(\sigma)} \frac{\delta}{\delta x^{\alpha}(\sigma)} & \equiv \frac{\delta}{\delta \sigma^{\alpha \beta}(\sigma)} \\
\frac{\delta}{\delta x^{\alpha}(\sigma)} & \equiv \dot{x}^{\beta}(\sigma) \frac{\delta}{\delta \sigma^{\alpha \beta}(\sigma)} .
\end{aligned}
$$




\section{A.3 The equations of QCD}

It is worth going further in our study of the gauge line. Let us then calculate the partial derivative (or path derivative [55]) with respect to $x_{\lambda}(\sigma)$ of Mandelstam's formula (1.265). We get:

$$
\begin{aligned}
\frac{\partial}{\partial x_{\lambda}(\sigma)} \frac{\partial}{\partial \dot{x}^{\beta}(\sigma)} \frac{\delta U(y, x ; \mathcal{C})}{\delta x^{\alpha}(\sigma)}= & -i g\left[\frac{\partial U(1, \sigma)}{\partial x_{\lambda}(\sigma)} G_{\alpha \beta}(\sigma) U(\sigma, 0)+U(1, \sigma) G_{\alpha \beta}(\sigma) \frac{\partial U(\sigma, 0)}{\partial x_{\lambda}(\sigma)}\right. \\
& \left.+U(1, \sigma) \frac{\partial G_{\alpha \beta}(\sigma)}{\partial x_{\lambda}(\sigma)} U(\sigma, 0)\right] .
\end{aligned}
$$

On the other hand, from (1.263), one can infer the actions of $\frac{\delta}{\delta x_{\lambda}(\sigma)}$ on $U(1, \sigma)$ and $U(\sigma, 0)$ :

$$
\left\{\begin{array}{l}
\frac{\partial U(1, \sigma)}{\partial x_{\lambda}(\sigma)}=i g P U(1, \sigma) A^{\lambda}(\sigma) \\
\frac{\partial U(\sigma, 0)}{\partial x_{\lambda}(\sigma)}=-i g P A^{\lambda}(\sigma) U(\sigma, 0)
\end{array}\right.
$$

since contributes, in every case, only one boundary term. Eq.(1.268) becomes finally:

$$
\frac{\partial}{\partial x_{\lambda}(\sigma)} \frac{\partial}{\partial \dot{x}^{\beta}(\sigma)} \frac{\delta U(y, x ; \mathcal{C})}{\delta x^{\alpha}(\sigma)}=-i g U(1, \sigma)\left(\frac{\partial}{\partial x_{\lambda}(\sigma)} G_{\alpha \beta}(\sigma)+i g\left[A^{\lambda}(\sigma), G_{\alpha \beta}(\sigma)\right]\right) U(\sigma, 0)
$$

where one recognizes the expression of the covariant derivative:

$$
\frac{\partial}{\partial x_{\lambda}(\sigma)} \frac{\partial}{\partial \dot{x}^{\beta}(\sigma)} \frac{\delta U(y, x ; \mathcal{C})}{\delta x^{\alpha}(\sigma)}=-i g U(1, \sigma) D^{\lambda} G_{\alpha \beta}(\sigma) U(\sigma, 0)
$$

From this equation, it is possible to obtain two fundamental results:

- by cyclic permutation over all the Lorentz indices, we get with (1.266):

$$
\begin{array}{r}
\left(\frac{\partial}{\partial x_{\lambda}(\sigma)} \frac{\delta}{\delta \sigma^{\alpha \beta}(\sigma)}+\frac{\partial}{\partial x_{\beta}(\sigma)} \frac{\delta}{\delta \sigma^{\lambda \alpha}(\sigma)}+\frac{\partial}{\partial x_{\alpha}(\sigma)} \frac{\delta}{\delta \sigma^{\beta \lambda}(\sigma)}\right) U(y, x ; \mathcal{C})= \\
-i g U(1, \sigma)\left(D^{\lambda} G_{\alpha \beta}(\sigma)+D^{\beta} G_{\lambda \alpha}(\sigma)+D^{\alpha} G_{\beta \lambda}(\sigma)\right) U(\sigma, 0)
\end{array}
$$

which is nothing else than the Bianchi identity of Yang-Mills theory. The gauge line satisfies thus the following constraint:

$$
\frac{\partial}{\partial x_{\lambda}(\sigma)} \frac{\delta U(y, x ; \mathcal{C})}{\delta \sigma^{\alpha \beta}(\sigma)}+(\text { cyclic permutation })=0
$$

- by contracting in (1.271) the Lorentz indices $\lambda$ and $\alpha$, we find:

$$
\frac{\partial}{\partial x_{\alpha}(\sigma)} \frac{\delta U(y, x ; \mathcal{C})}{\delta \sigma^{\alpha \beta}(\sigma)}=-i g U(1, \sigma) D^{\alpha} G_{\alpha \beta}(\sigma) U(\sigma, 0)
$$

In this case, we recognize on the r.h.s the first term $D^{\alpha} G_{\alpha \beta}$ in the equation of motion of the Yang-Mills gauge field. 


\section{A.4 The Wilson loop: the colour trace of a closed gauge line}

When the gauge line endpoints of coordinates $x$ and $y$ coincide in (1.245), $x(\sigma=0)=$ $x(\sigma=1)$ and we obtain a closed gauge line $U(x, x ; \mathcal{C})$. Then, the trace on the colour space gives the so-called Wilson loop $\phi(\mathcal{C})$ :

$$
\phi(\mathcal{C}) \equiv \operatorname{Tr}_{c} U(x, x ; \mathcal{C})=\operatorname{Tr}_{c} P e^{-i g \oint_{\mathcal{C}} A_{\mu}(x) d x^{\mu}} .
$$

Let us pointing out that the Wilson loop does not depend in (1.275) on the point $x$ from which is parametrized the loop $\mathcal{C}$ because of the colour trace $\operatorname{Tr}_{c}$. Moreover, it is a manifestly gauge invariant functional. Indeed, under a gauge transformation, we have:

$$
U(x, x ; \mathcal{C}) \rightarrow U^{\prime}(x, x ; \mathcal{C})=\Omega(x) U(x, x ; \mathcal{C}) \Omega^{\dagger}(x)
$$

so that

$$
\begin{aligned}
\phi(\mathcal{C}) \rightarrow \phi^{\prime}(\mathcal{C}) & =\operatorname{Tr}_{c} U^{\prime}(x, x ; \mathcal{C}) \\
& =\operatorname{Tr}_{c}\left(\Omega(x) U(x, x ; \mathcal{C}) \Omega^{\dagger}(x)\right) \\
\phi^{\prime}(\mathcal{C}) & =\operatorname{Tr}_{c} U(x, x ; \mathcal{C})=\phi(\mathcal{C})
\end{aligned}
$$

by invoking the invariance of the trace under a cyclic permutation.

\section{A.5 The Migdal-Makeenko equation and the loop equation}

When one reformulates a non-Abelian gauge theory as QCD into the loop space [51, 56, $57,58]$, all the references to the gauge fields, gauge transformations, gauge-fixing terms, ghosts and so on and so forth are discarded and only remain gauge invariant functionals. The observables are then expressed in terms of such functionals and the equations of motion of the gauge fields describing QCD dynamics (without fermions) are replaced by functional equations. The loop space formalism being gauge invariant, the properties of the gauge group become functional constraints. Examples of such constraints are Mandelstam's constraints [59, 60], among which we have for instance:

- the reparametrization invariance:

$$
\phi(\mathcal{C})=\phi\left(\mathcal{C}^{\prime}\right)
$$

where $\mathcal{C}=\{\sigma \rightarrow x(\sigma)\}$ and $\mathcal{C}^{\prime}=\left\{\sigma^{\prime}=f(\sigma) \rightarrow x\left(\sigma^{\prime}\right)\right\}$ with $f(0)=0$ and $f(1)=1$.

- The reversal relation:

$$
\phi(\mathcal{C})=\phi\left(\mathcal{C}^{\prime}\right)^{-1}
$$

with $x\left(\sigma^{\prime}\right)=x(1-\sigma)$. 
Although this program gives rise to strong difficulties, a certain number of issues have been resolved. For example, it has been shown that the Yang-Mills equation of motion becomes, in loop space, a functional equation satisfied by the Wilson loop, the so-called Migdal-Makeenko equation [53, 61]:

$$
\frac{\partial}{\partial x^{\mu}} \frac{\delta}{\delta \sigma_{\mu \nu}(x)}\langle\phi(\mathcal{C})\rangle_{A}=-\frac{i g^{2}}{2} \oint_{\mathcal{C}} d y^{\nu} \delta^{4}(x-y)\left\langle\phi\left(\mathcal{C}_{y x}\right) \phi\left(\mathcal{C}_{x y}\right)-\frac{1}{N_{c}} \phi(\mathcal{C})\right\rangle_{A},
$$

the averaging being defined in the path-integral formalism. Before studying its most important properties, let us roughly check its derivation. For this, we consider the equation of motion (1.274) derived from Mandelstam's formula:

$$
\frac{\partial}{\partial x^{\mu}(\sigma)} \frac{\partial}{\partial \dot{x}_{\nu}(\sigma)} \frac{\delta \phi(\mathcal{C})}{\delta x_{\mu}(\sigma)}=-i g U(1, \sigma) D_{\mu} G^{\mu \nu}(\sigma) U(\sigma, 0)
$$

or, in tensorial notation:

$$
\frac{\partial}{\partial x^{\mu}(\sigma)} \frac{\partial}{\partial \dot{x}_{\nu}(\sigma)} \frac{\delta \phi(\mathcal{C})}{\delta x_{\mu}(\sigma)}=-\frac{i g}{\sqrt{2}} U_{c}^{a}(1, \sigma) D_{\mu_{d}^{c}}^{\mu} G_{e}^{d}(\sigma) U_{a}^{e}(\sigma, 0)
$$

where we have defined $\left(G_{\mu \nu}\right)_{b}^{a}=\left(G_{\mu \nu}^{i} \frac{\lambda^{i}}{2}\right)_{b}^{a} \equiv \frac{1}{\sqrt{2}} G_{\mu \nu b}^{a}$. In QCD, the equation of motion of the gluon field is:

$$
D_{\mu} G^{\mu \nu}(x)=j_{F}^{\nu}(x)+j_{g f}^{\nu}(x)+j_{F P}^{\nu}(x)
$$

with $j_{F}^{\nu}(x)$ the fermionic current and $j_{g f}^{\nu}(x)$ and $j_{F P}^{\nu}(x)$ the currents associated with the gauge-fixing term and the Faddeev-Popov ghosts respectively. If we remark that the functional derivative of a path integral vanishes:

$$
\frac{\delta}{\delta A}\langle Q[A]\rangle_{A}=0
$$

for any functional $Q[A]$ of the gauge field, then the equation of motion reads in the path-integral formalism as:

$$
\left\langle i \frac{\delta Q}{\delta A}\right\rangle_{A}=\left\langle\frac{\delta S}{\delta A} Q\right\rangle_{A}
$$

Without external fermionic source, $j_{F}^{\nu}(x)$ vanishes. Moreover, since the Wilson loop $\phi(\mathcal{C})$ is gauge invariant, the currents $j_{g f}^{\nu}(x)$ and $j_{F P}^{\nu}(x)$ cancel out each other (the Slanov-TaylorWard-Takahashi identity shows that $j_{g f}^{\nu}(x)+j_{F P}^{\nu}(x)=0$ in the path-integral formalism [61]). Therefore, we are led to write out:

$$
D_{\mu} G^{\mu \nu}(x) \equiv i \frac{\delta}{\delta A_{\nu}(x)}
$$

which should be understood in the weak sense, namely in terms of mean values. As a result, the equation (1.282) becomes in the path-integral formalism:

$$
\frac{\partial}{\partial x^{\mu}(\sigma)} \frac{\partial}{\partial \dot{x}_{\nu}(\sigma)} \frac{\delta\langle\phi(\mathcal{C})\rangle_{A}}{\delta x_{\mu}(\sigma)}=-\frac{i g}{\sqrt{2}}\left\langle P\left(i \frac{\delta}{\delta A_{\nu_{c}^{e}}^{e}(\sigma)}\right) U_{c}^{a}(1, \sigma) U_{a}^{e}(\sigma, 0)\right\rangle_{A}
$$


Expanding as before (1.249) the gauge line $U_{c}^{a}(1, \sigma)$ as a power series, the contribution of the order $O\left(g^{n}\right)$ involves the product of $n$ gauge fields:

$$
U_{c}^{a}(1, \sigma) U_{a}^{e}(\sigma, 0) \simeq A_{\mu_{1} c_{1}}^{a}\left(\sigma_{1}\right) A_{\mu_{2} c_{2}}^{c_{1}}\left(\sigma_{2}\right) A_{\mu_{3} c_{3}}^{c_{2}}\left(\sigma_{3}\right) A_{\mu_{4} c_{4}}^{c_{3}}\left(\sigma_{4}\right) \ldots A_{\mu_{n}}^{c_{n-1}}\left(\sigma_{n}\right) U_{a}^{e}(\sigma, 0)
$$

For the sake of argument, let us consider the contribution of the field $A_{\mu_{4} c_{4}}^{c_{3}}\left(\sigma_{4}\right)$. The functional derivative with respect to $\delta / \delta A_{\nu_{c}}^{e}(\sigma)$ gives:

$$
\begin{gathered}
\delta_{\mu_{4}}^{\nu} \delta^{4}\left(x\left(\sigma_{4}\right)-x(\sigma)\right)\left\{A_{\mu_{1} c_{1}}^{a}\left(\sigma_{1}\right) A_{\mu_{2} c_{2}}^{c_{1}}\left(\sigma_{2}\right) A_{\mu_{3} e}^{c_{2}}\left(\sigma_{3}\right)\left[A_{\mu_{5} c_{5}}^{c}\left(\sigma_{5}\right) \ldots A_{\mu_{n} c}^{c_{n-1}}\left(\sigma_{n}\right)\right]\right. \\
\left.-\frac{1}{N_{c}} A_{\mu_{1} c_{1}}^{a}\left(\sigma_{1}\right) A_{\mu_{2} c_{2}}^{c_{1}}\left(\sigma_{2}\right) A_{\mu_{3} c_{3}}^{c_{2}}\left(\sigma_{3}\right) A_{\mu_{5} c_{5}}^{c_{3}}\left(\sigma_{5}\right) \ldots A_{\mu_{n} e}^{c_{n-1}}\left(\sigma_{n}\right)\right\} U_{a}^{e}(\sigma, 0)
\end{gathered}
$$

according to

$$
\frac{\delta A_{\mu_{b}}^{a}(\sigma)}{\delta A_{d}^{\nu^{c}}\left(\sigma^{\prime}\right)}=\eta_{\mu \nu}\left(\delta_{c}^{a} \delta_{b}^{d}-\frac{1}{N_{c}} \delta_{b}^{a} \delta_{d}^{c}\right) \delta^{4}\left(x(\sigma)-x\left(\sigma^{\prime}\right)\right)
$$

where the tensorial structure on the colour indices comes from the fact that the gluon fields are traceless. Because of the delta function $\delta^{4}(x-y)$ in $(1.289)$, the points $x \equiv x(\sigma)$ and $y \equiv x\left(\sigma_{4}\right)$ coincide in space-time but can be different in loop space as they are associated with different values of parameter. In this case, only contributes the first term in (1.289) which corresponds to the creation of an internal loop at the point $x$ of $\mathcal{C}$ :

$$
\left[A_{\mu_{5} c_{5}}^{c}\left(\sigma_{4}\right) \ldots A_{\mu_{n} c}^{c_{n-1}}(\sigma)\right] .
$$

Thus, this contribution gives rise in (1.280) to the product of the two Wilson loops $\phi\left(\mathcal{C}_{y x}\right)$ and $\phi\left(\mathcal{C}_{x y}\right)$.

On the other hand, when the parameters are equal $\sigma=\sigma_{4}$, only contributes the second term in (1.289) which corresponds to only one Wilson loop of contour $\mathcal{C}$. Finally, we get the Migdal-Makeenko equation:

$$
\frac{\partial}{\partial x^{\mu}} \frac{\delta}{\delta \sigma_{\mu \nu}}\langle\phi(\mathcal{C})\rangle_{A}=-\frac{i g^{2}}{2} \oint_{\mathcal{C}} d y^{\nu} \delta^{4}(x-y)\left\langle\phi\left(\mathcal{C}_{y x}\right) \phi\left(\mathcal{C}_{x y}\right)-\frac{1}{N_{c}} \phi(\mathcal{C})\right\rangle_{A}
$$

where the current

$$
j^{\nu}(x) \equiv-\frac{i g^{2}}{2} \oint_{\mathcal{C}} d y^{\nu} \delta^{4}(x-y)\left\langle\phi\left(\mathcal{C}_{y x}\right) \phi\left(\mathcal{C}_{x y}\right)-\frac{1}{N_{c}} \phi(\mathcal{C})\right\rangle_{A}
$$

is conserved, $\partial_{\nu} j^{\nu}=0$, thanks to the antisymmetry property of the surface derivative $\delta / \delta \sigma_{\mu \nu}(x)$ in the exchange $\mu \leftrightarrow \nu$.

Let us define the following gauge invariant one- and two-loop functionals:

$$
\begin{aligned}
W(\mathcal{C}) & \equiv \frac{1}{N_{c}}\langle\phi(\mathcal{C})\rangle_{A} \\
W_{2}\left(\mathcal{C}_{1}, \mathcal{C}_{2}\right) & \equiv\left\langle\frac{1}{N_{c}} \phi\left(\mathcal{C}_{1}\right) \frac{1}{N_{c}} \phi\left(\mathcal{C}_{2}\right)\right\rangle_{A}
\end{aligned}
$$


as the vacuum expectation values, respectively of the Wilson loop $\phi(\mathcal{C})$ and of the product of two loops $\phi\left(\mathcal{C}_{1}\right) \phi\left(\mathcal{C}_{2}\right)$ (up to normalization factors in $1 / N_{c}$ ). The Migdal-Makeenko equation takes then the following form:

$$
\frac{\partial}{\partial x^{\mu}} \frac{\delta}{\delta \sigma_{\mu \nu}(x)} W(\mathcal{C})=-i \lambda \oint_{\mathcal{C}} d y^{\nu} \delta^{4}(x-y)\left(W_{2}\left(\mathcal{C}_{y x}, \mathcal{C}_{x y}\right)-\frac{1}{N_{c}^{2}} W(\mathcal{C})\right)
$$

with $\lambda \equiv g^{2} N_{c} / 2$. Eq. (1.296) involves the three loops $\mathcal{C}, \mathcal{C}_{y x}$ (oriented from $x$ to $y$ ) and $\mathcal{C}_{x y}$ (oriented from $y$ to $x$ ) such that $\mathcal{C}_{y x} \cup \mathcal{C}_{c y}=\mathcal{C}$ and is, consequently, a non-linear and non-closed functional equation: the one-loop functional $W(\mathcal{C})$ (also called, roughly speaking, Wilson loop) is related to the two-loop functional $W_{2}\left(\mathcal{C}_{y x}, \mathcal{C}_{y x}\right)$. The MigdalMakeenko equation is the first equation of an infinite set of functional equations relating the derivatives of $n$-loop functionals to the integrals of $(n-1)-, n-$ and $(n+1)$-loop functionals.

Most of the time, one finds, in the literature, the Migdal-Makeenko equation written in the Euclidean space-time $E^{4}$ with the metric tensor $\delta_{\mu \nu}=\operatorname{diag}(1,1,1,1)$. In this case, the Migdal-Makeenko equation (1.296) becomes:

$$
\frac{\partial}{\partial x_{\mu}} \frac{\delta}{\delta \sigma_{\mu \nu}(x)} W(\mathcal{C})=\lambda \oint_{\mathcal{C}} d y^{\nu} \delta^{4}(x-y)\left(W_{2}\left(\mathcal{C}_{y x}, \mathcal{C}_{x y}\right)-\frac{1}{N_{c}^{2}} W(\mathcal{C})\right) .
$$

There are two cases in which (1.297) can be simplified : in the Abelian case where $N_{c}=1$ and in the 't Hooft limit where $N_{c} \rightarrow \infty$ with $\lambda$ finite.

- When $N_{c}=1$, one deals with an Abelian $U(1)$ gauge theory with coupling constant $g^{\prime}$ [61]. The gauge field is no longer a matrix such that

$$
\frac{\delta A_{\mu}(\sigma)}{\delta A^{\nu}\left(\sigma^{\prime}\right)}=\delta_{\mu \nu} \delta^{4}\left(x(\sigma)-x\left(\sigma^{\prime}\right)\right)
$$

in (1.287). As a result, only the first term in (1.289) (without colour indices) contributes and only remains on the r.h.s of (1.297) the two-loop functional $W_{2}$. Moreover, the square matrices $\phi\left(\mathcal{C}_{x y}\right)$ and $\phi\left(\mathcal{C}_{y x}\right)$ of order $N_{c}$ become simple operators and $W_{2}\left(\mathcal{C}_{1}, \mathcal{C}_{2}\right)$ reduces to the Wilson loop $W(\mathcal{C})$. At the end of the day, the Abelian Migdal-Makeenko equation takes the form:

$$
\frac{\partial}{\partial x_{\mu}} \frac{\delta}{\delta \sigma_{\mu \nu}(x)} W(\mathcal{C})=g^{\prime 2} \oint_{\mathcal{C}} d y^{\nu} \delta^{4}(x-y) W(\mathcal{C})
$$

which has the solution:

$$
W(\mathcal{C})=e^{-\frac{g^{\prime 2}}{2} \oint_{\mathcal{C}} \oint_{\mathcal{C}} d x_{\mu} d y_{\nu} D_{\mu \nu}(x-y)}
$$

where $D_{\mu \nu}(x-y)$ is the coulombic propagator of the Abelian gauge field $A_{\mu}$ :

$$
D_{\mu \nu}(x-y)=\frac{\delta_{\mu \nu}}{4 \pi^{2}} \frac{1}{(x-y)^{2}}
$$


in the Feynman gauge.

- In the 't Hooft limit where $N_{c} \rightarrow \infty$ with $\lambda$ finite [62,63], one can invoke the following factorization property:

$$
W_{2}\left(\mathcal{C}_{1}, \mathcal{C}_{2}\right)=W\left(\mathcal{C}_{1}\right) W\left(\mathcal{C}_{2}\right)+O\left(\frac{1}{N_{c}^{2}}\right)
$$

in order to simplify (1.297). This property has been demonstrated at any order of Perturbation Theory: at each order, only remain, in the 't Hooft limit, the planar diagrams in which every gluon is emitted and absorbed by the same Wilson loop (in general, a gauge invariant operator). The diagrams connected by gluon exchanges (namely, when a gluon emitted by a Wilson loop is reabsorbed by another Wilson loop) are suppressed in $1 / N_{c}^{2}$. The factorization property has also been proved in non-perturbative regime. The equation (1.297) thus becomes:

$$
\frac{\partial}{\partial x_{\mu}} \frac{\delta}{\delta \sigma_{\mu \nu}(x)} W(\mathcal{C})=\lambda \oint_{\mathcal{C}} d y^{\nu} \delta^{4}(x-y) W\left(\mathcal{C}_{y x}\right) W\left(\mathcal{C}_{x y}\right) .
$$

This is the so-called loop equation which is a non-linear but closed functional equation since only one-loop functionals contribute.

It is worth summarizing the theory in loop space when $N_{c} \rightarrow \infty$ with $\lambda$ finite. We have the following two equations:

- the loop equation:

$$
\frac{\partial}{\partial x_{\mu}} \frac{\delta}{\delta \sigma_{\mu \nu}(x)} W(\mathcal{C})=\lambda \oint_{\mathcal{C}} d y^{\nu} \delta^{4}(x-y) W\left(\mathcal{C}_{y x}\right) W\left(\mathcal{C}_{x y}\right),
$$

- the Bianchi identity:

$$
\frac{\partial}{\partial x_{\alpha}} \frac{\delta}{\delta \sigma_{\mu \nu}(x)} W(\mathcal{C})+(\text { cyclic permutation })=0 .
$$

The initial condition when the loop shrinks into a point is:

$$
W\left(\mathbb{I}_{\mathcal{C}}\right)=1
$$

where $\mathbb{I}_{\mathcal{C}}=\{\sigma \rightarrow x(\sigma)=x\}$ is the identity.

Finally, we conclude this section by recapitulating the correspondence between the Yang-Mills theory and its formulation in loop space:

- the equation of motion:

$$
D_{\mu} G_{\mu \nu}(x)=0 \Rightarrow \frac{\partial}{\partial x_{\mu}} \frac{\delta}{\delta \sigma_{\mu \nu}(x)} W(\mathcal{C})=\lambda \oint_{\mathcal{C}} d y^{\nu} \delta^{4}(x-y)\left(W_{2}-\frac{1}{N_{c}^{2}} W\right),
$$

- the Bianchi identity:

$D_{\alpha} G_{\mu \nu}(x)+($ cyclic permutation $)=0 \Rightarrow \frac{\partial}{\partial x_{\alpha}} \frac{\delta}{\delta \sigma_{\mu \nu}(x)} W(\mathcal{C})+($ cyclic permutation $)=0$. 


\section{A.6 The renormalization of the Wilson loop}

When one evaluates, in Perturbation Theory, line integrals in the Wilson loop, one meets with singularities which have to be regularized. And yet, the renormalization of the Wilson loop is still far from being trivial since it behaves as a non-local object.

- When the loop $\mathcal{C}$ is smooth (i.e. differentiable) and simple (i.e. without nodes), the leading perturbative contribution to $W(\mathcal{C})$ corresponds to the one-gluon exchange (contribution of order $O\left(g^{2}\right)$ ). It diverges linearly in $\frac{\pi}{a} L(\mathcal{C})$ where $L(\mathcal{C})$ is the length of the contour $\mathcal{C}$ and $a$ is a short distance cutoff. In 1980, Polyakov [52], Dotsenko and Vergeles [64] proved that the Wilson loop is multiplicatively renormalizable for such a smooth and simple contour: at any order, linear divergences appear which can be gathered into a common factor $Z=e^{\text {const. } \frac{L(\mathcal{C})}{a}}$ where const. is of order $O(1)$. Beyond the second order, it also appears logarithmic divergences which can be then absorbed by renormalization of the strong coupling constant $g$. At the end of the day, we obtain:

$$
W(g, \mathcal{C})=Z W_{R}\left(g_{R}, \mathcal{C}\right)
$$

where $W_{R}\left(g_{R}, \mathcal{C}\right)$ is finite, provided that it is expressed in terms of the renormalized strong coupling constant $g_{R}$. The physical meaning of the linear divergence $Z$ is easily explained if we consider $\ln (W(\mathcal{C}))$ as the effective action of a test particle constrained to move along $\mathcal{C}$ : the factor $Z$ disappears with the renormalization of the mass.

- When the contour is no longer smooth but has a cusp of angle $\gamma$, the renormalization still remains multiplicative $[61,65]$ :

$$
W(g, \mathcal{C})=Z(\gamma) \tilde{W}(g, \mathcal{C})
$$

where $\tilde{W}(g, \mathcal{C})$ refers to the Wilson loop on the l.h.s of $(1.309)$. The factor $Z(\gamma)$ is an additional logarithmic divergence which depends locally on the loop $\mathcal{C}$ (at the vicinity of the cusp). Thus, this anomalous divergence cannot be absorbed by renormalization of $g$.

- Let us consider a loop $\mathcal{C}$ which intersects one or several times at the same space-time point and which is smooth everywhere else. At the beginning of the eighties, Brandt, Gocksch, Sato and Neri [61, 65] showed that the Wilson loop cannot be renormalized alone in this case. It must be renormalized by mixing with all the other loops $W_{n_{i}}\left(\left\{\mathcal{C}_{j}^{i}\right\}\right) \equiv$ $W^{i}\left(\left\{\mathcal{C}_{j}^{i}\right\}\right)$ where $i=1,2, \ldots, I$ ( $I$ is the number of sets) and $j=1,2, \ldots, n_{i}\left(n_{i}\right.$ is the number of loops $\mathcal{C}_{j}^{i}$ in the set $i$ ). Given a set $i$, the $n_{i}$ loops $\mathcal{C}_{j}^{i}$, s must be identical to the corresponding sections of $\mathcal{C}$, both in space-time (every $\mathcal{C}_{j}^{i}$ draws the same path than $\mathcal{C}$ ) and in direction (every $\mathcal{C}_{j}^{i}$ is oriented as $\mathcal{C}$ ).

In order to illustrate quickly the mechanism, let us consider the simplest case of a loop with only one node (there is thus only one independent angle $\gamma$ ). To the contour $\mathcal{C}$ is associated two sets: $\left\{\mathcal{C}_{1}^{1}\right\}$ which is nothing else than $\mathcal{C}$ when the path ordering prescription 
$P$ is taken into account and $\left\{\mathcal{C}_{1}^{2}, \mathcal{C}_{2}^{2}\right\}$ corresponding to the two sections of the loop on either side of the crossing point. The set of the Wilson loops $W^{1}\left(\mathcal{C}_{1}^{1}\right)=W(\mathcal{C})$ and $W^{2}\left(\mathcal{C}_{1}^{2}, \mathcal{C}_{2}^{2}\right)$ mix then together under renormalization through the square matrix of order $2 Z(\gamma)$.

The generalization to more complicated loops gives:

$$
W^{i}\left(g,\left\{\mathcal{C}_{j}^{i}\right\}\right)=\sum_{k=1}^{I} Z^{i k}(\gamma) \tilde{W}^{k}\left(g,\left\{\mathcal{C}_{l}^{k}\right\}\right)
$$

where $Z(\gamma)$ is a matrix of order $I$ depending on all the independent angles at the considered node.

\section{A.7 The Wilson loop in $\mathrm{QCD}_{2}$}

Since 't Hooft's seminal paper in 1974 [63], numerous physicists have sought to understand the properties of QCD in $(1+1)$-dimensional space-time (see, e.g. [66, 67]). As a matter of fact, the Migdal-Makeenko equation turns out to be, in this case, exactly solvable in the large $N_{c}$ limit. In the sequel, I will essentially refer to the review [53]; the interested reader could fruitfully read the pioneering works $[68,69]$ where is detailed the resolution.

Within the loop space formalism, the theory is manifestly gauge invariant: let us choose the axial gauge $n \cdot A=0$. In $(1+1)$-dimensional space-time, $n^{\mu}=(0,1)$, thus $A_{1}$ vanishes and only remains $A_{0}$. The interest of the axial gauge is twofold: first of all, it discards the gluon self-interactions (the commutator $\left[A_{\mu}, A_{\nu}\right]$ in the strength field tensor being then equal to zero). Secondly, the ghosts decouple from the theory and can be ignored. As a result, $\mathrm{QCD}_{2}$ in the axial gauge looks like, at first sight, an Abelian theory.

From the diagrammatic point of view, the Wilson loop $W(\mathcal{C})$ sums, in the 't Hooft limit, the disconnected planar diagrams, i.e. those which are only made up of free propagators (in the axial gauge, there are not three- or four-gluon interactions). In a first time, we consider the simplest case of a smooth and simple loop $\mathcal{C}$. One finds $\left(\lambda=g^{2} N_{c}\right)$ :

$$
W(\mathcal{C})=e^{-\frac{\lambda}{2} \oint_{\mathcal{C}} \oint_{\mathcal{C}} d x_{\mu} d y_{\nu} D_{\mu \nu}(x-y)}
$$

which strongly mimics the Abelian solution (1.300). The gluon propagator

$$
D_{\mu \nu}(x-y) \equiv \frac{1}{N_{c}} \operatorname{Tr}_{c}\left\langle 0\left|A_{\mu}(x) A_{\nu}(y)\right| 0\right\rangle
$$

reads in the axial gauge as

$$
D_{\mu \nu}(x-y)=\frac{1}{2} \delta_{\mu 0} \delta_{\nu 0}\left|x_{1}-y_{1}\right| \delta\left(x_{0}-y_{0}\right) .
$$

Because of the delta function $\delta\left(x_{0}-y_{0}\right)$ involving the time coordinates, the interaction is instantaneous. Although this result is valid in general, it is easier to demonstrate it 
in Perturbation Theory. For this, we expand the (non-renormalized) Wilson loop $W(\mathcal{C})$ in powers of $g[53,65]$ (we are unaware here of the existence of regularization schemes required in order to deal with well-defined calculations):

$$
W(\mathcal{C})=1+\sum_{n=2}^{\infty}(-i g)^{n} \oint_{\mathcal{C}} d x_{1}^{\mu_{1}} \times \ldots \times \oint_{\mathcal{C}} d x_{n}^{\mu_{n}} \theta(\mathcal{C} ; 1, \ldots, n) G_{\mu_{1} \ldots \mu_{n}}^{(n)}\left(x_{1}, \ldots, x_{n}\right)
$$

The prescription $\theta(\mathcal{C} ; 1, \ldots, n)$ puts in order the points $x_{1}, \ldots, x_{n}$ along the loop $\mathcal{C}$ and $G^{(n)}$ is the Green function with $n$ external legs attached to $\mathcal{C}$ :

$$
G_{\mu_{1} \ldots \mu_{n}}^{(n)}\left(x_{1}, \ldots, x_{n}\right) \equiv \frac{1}{N_{c}} \operatorname{Tr}_{c}\left\langle 0\left|A_{\mu_{1}}\left(x_{1}\right) \ldots A_{\mu_{n}}\left(x_{n}\right)\right| 0\right\rangle
$$

satisfying the normalization condition $G^{(0)}(0)=1$. Since the theory behaves, in the axial gauge, as an Abelian theory, the gauge fields do not interact each other such that $n$ must be even $n=2 k$. The expansion (1.315) then becomes:

$$
\begin{aligned}
W(\mathcal{C})= & 1+\sum_{\substack{k=1 \\
\infty}}^{\infty}(-i g)^{2 k} \oint_{\mathcal{C}} d x_{1}^{\mu_{1}} \times \ldots \times \oint_{\mathcal{C}} d x_{2 k}^{\mu_{2 k}} \theta(\mathcal{C} ; 1, \ldots, 2 k) \\
& \times N_{c}^{k-1} D_{\mu_{1} \mu_{2}}\left(x_{1}-x_{2}\right) \ldots D_{\mu_{2 k-1} \mu_{2 k}}\left(x_{2 k-1}-x_{2 k}\right) .
\end{aligned}
$$

At the lowest order, we have:

$$
W(\mathcal{C})=1+(-i g)^{2} \oint_{\mathcal{C}} d x_{1}^{\mu_{1}} \oint_{\mathcal{C}} d x_{2}^{\mu_{2}} \theta(\mathcal{C} ; 1,2) N_{c} D_{\mu_{1} \mu_{2}}\left(x_{1}-x_{2}\right)+O\left(g^{4}\right)
$$

Moreover, $D_{\mu_{1} \mu_{2}}\left(x_{1}-x_{2}\right)=D_{\mu_{2} \mu_{1}}\left(x_{2}-x_{1}\right)$ and $\theta(\mathcal{C} ; 1,2)+\theta(\mathcal{C} ; 2,1)=1$ such that

$$
W(\mathcal{C})=1-\frac{g^{2} N_{c}}{2} \oint_{\mathcal{C}} d x_{1}^{\mu_{1}} \oint_{\mathcal{C}} d x_{2}^{\mu_{2}} D_{\mu_{1} \mu_{2}}\left(x_{1}-x_{2}\right)+O\left(g^{4}\right) .
$$

One can generalize this mechanism at any order to obtain the solution (1.312) by taking the exponential of the expansion of $W(\mathcal{C})$.

For a smooth and simple loop $\mathcal{C}$, the exponential factor in (1.312) is easily evaluated:

$$
\oint_{\mathcal{C}} \oint_{\mathcal{C}} d x_{\mu} d y_{\nu} D_{\mu \nu}(x-y)=A(\mathcal{C})
$$

where $A(\mathcal{C})$ represents the area of the (plane) surface with $\mathcal{C}$ as boundary. The Wilson loop takes then the form:

$$
W(\mathcal{C})=e^{-\frac{\lambda}{2} A(\mathcal{C})}
$$

which is the area law in $\mathrm{QCD}_{2}$. It is worth pointing out that this behaviour (1.321) is valid in the non-Abelian case as well, the difference appearing only for more complicated loops. So, let us focus on a contour having one node. We have then two configurations: the first configuration gives similar results in the Abelian and non-Abelian theories: $A(\mathcal{C})=$ 
$A_{1}+A_{2}$ and $W(\mathcal{C})=e^{-\frac{\lambda}{2}\left(A_{1}+A_{2}\right)}$. The second configuration gives $A(\mathcal{C})=A_{1}+4 A_{2}$ but $W(\mathcal{C})=e^{-\frac{\lambda}{2}\left(A_{1}+4 A_{2}\right)}$ in the Abelian case whereas $W(\mathcal{C})=\left(1-\lambda A_{2}\right) e^{-\frac{\lambda}{2}\left(A_{1}+2 A_{2}\right)}$ in the non-Abelian case where contribute only the planar diagrams in the 't Hooft limit. The generalization for any loop gives in $\mathrm{QCD}_{2}$ :

$$
W(\mathcal{C})=\sum_{i} P_{i}\left(A_{1}, \ldots, A_{n}\right)
$$

where the $P_{i}$ 's are exponential functions of the $n$ surfaces of areas $A_{i}(i=1, \ldots, n)$ which make up $A(\mathcal{C})$.

\section{References}

[1] J. M. Maldacena, The Large N limit of superconformal field theories and supergravity, Adv. Theor. Math. Phys. 2 (1998) 231 [Int. J. Theor. Phys. 38 (1999) 1113] [arXiv:hep-th/9711200].

[2] E. Witten, Anti-de Sitter space and holography, Adv. Ther. Math. Phys. 2 (1998) 253 [arXiv:hep-th/9802150].

[3] S. S. Gubser, I. R. Klenanov \& A. M. Polyakov, Gauge theory correlators from noncritical string theory, Phys. Lett. B428 (1998) 105 [arXiv:hep-th/9802109].

[4] J. M. Maldacena, Wilson loops in large $N$ field theories, Phys. Rev. Lett. 80 (1998) 4859 [arXiv:hep-th/9803002].

[5] O. Aharony, S. S. Gubser, J. M. Maldacena, H. Ooguri \& Y. Oz, Large N field theories, string theory and gravity, Phys. Rept. 323 (2000) 183 [arXiv:hep-th/9905111]; D. Mateos, String Theory and Quantum Chromodynamics, Class. Quant. Grav. 24 (2007) S713 [arXiv:hep-th/0709.1523].

[6] G. T. Horowitz \& A. Strominger, Black strings and P-branes, Nucl. Phys. B360 (1991) 197.

[7] A. Strominger, Black hole entropy from near horizon microstates, JHEP 9802 (1998) 009 [arXiv:hep-th/9712251]; V. Balasubramanian \& F. Larsen, Near horizon geometry and black holes in four-dimensions, Nucl. Phys. B528 (1998) 229 [arXiv:hepth/9802198]; W. Mueck \& K. S. Viswanathan, Conformal field theory correlators from classical scalar field theory on $A d S_{d+1}$, Phys. Rev. D58 (1998) 041901 [arXiv:hepth/9804035]; D. Z. Freedman, S. D. Mathur, A. Matusis \& L. Rastelli, Correlation functions in the $C F T_{d} / A d S_{d+1}$ correspondence Nucl. Phys. B546 (1999) 96 
[arXiv:hep-th/9804058]; S. Lee, S. Minwalla, M. Rangamani \& N. Seiberg, Three point functions of chiral operators in $D=4, \mathcal{N}=4 S Y M$ at large $N$, Adv. Theor. Math. Phys. 2 (1998) 697 [arXiv:hep-th/9806074]; H. Liu \& A.A. Tseytlin, On four point functions in the CFT/AdS correspondence, Phys. Rev. D59 (1999) 086002 [arXiv:hep-th/9807097]; E. D'Hoker \& D. Z. Freedman, Field theory tests for correlators in the AdS/CFT correspondence, Phys. Rev. D59 (1999) 045008 [arXiv:hepth/9807098].

[8] E. Witten, Anti-de Sitter space, thermal phase transition, and confinement in gauge theories, Adv. Theor. Math. Phys. 2 (1998) 505 [arXiv:hep-th/9803131].

[9] J. Polchinski \& M.J. Strassler, Hard scattering and gauge/string duality, Phys. Rev. Lett. 88 (2002) 031601 [arXiv:hep-th/0109174].

[10] J. Erlich, E. Katz, D.T. Son \& M.A. Stephanov, QCD and a holographic model of hadrons, Phys. Rev. Lett. 95 (2005) 261602 [arXiv:hep-ph/0501128].

[11] L. Da Rold \& A. Pomarol, Chiral symmetry breaking from five dimensional spaces, Nucl. Phys. B721 (2005) 79 [arXiv:hep-ph/0501218].

[12] L. Da Rold \& A. Pomarol, The scalar and pseudoscalar sector in a five-dimensional approach to chiral symmetry breaking, JHEP 0601 (2006) 157 [arXiv:hepph/0510268]; S. J. Brodsky \& G. F. de Teramond, Hadronic spectra and light-front wavefunctions in holographic QCD, Phys. Rev. Lett. 96 (2006) 201601 [arXiv:hepph/0602252]; S. J. Brodsky, Hadron Spectroscopy and Structure from AdS/CFT, Eur. Phys. J. A31 (2007) 638 [arXiv:hep-ph/0610115]; H. Boschi-Filho, N. R.F. Braga \& H. L. Carrion, Glueball Regge trajectories from gauge/string duality and the Pomeron, Phys. Rev. D73 (2006) 047901 [arXiv:hep-th/0507063]; H. Forkel, Holographic glueball structure, Phys. Rev. D78 (2008) 025001 [arXiv:hep-ph/0711.1179]; D. K. Hong, T. Inami \& H.-U. Yee, Baryons in AdS/QCD, Phys. Lett. B646 (2007) 165 [arXiv:hep-ph/0609270]; A. Pomarol \& A. Wulzer, Baryon Physics in Holographic QCD, Nucl. Phys. B809 (2009) 347 [arXiv:hep-ph/0807.0316].

[13] S. J. Brodsky \& G. F. de Teramond, Light-Front Dynamics and AdS/QCD Correspondence: The Pion Form Factor in the Space- and Time-Like Regions, Phys. Rev. D77 (2008) 056007 [arXiv:hep-ph/0707.3859]; H. R. Grigoryan \& A. V. Radyushkin, Form Factors and Wave Functions of Vector Mesons in Holographic QCD, Phys. Lett. B650 (2007) 421 [arXiv:hep-ph/0703069]; Pion form-factor in chiral limit of hard-wall AdS/QCD model, Phys. Rev. D76 (2007) 115007 [arXiv:hep-ph/0709.0500]; Anomalous Form Factor of the Neutral Pion in Extended AdS/QCD Model with 
Chern-Simons Term, Phys. Rev. D77 (2008) 115024 [arXiv:hep-ph/0803.1143]; H. J. Kwee \& R. F. Lebed, Pion form-factors in holographic QCD, JHEP 0801 (2008) 027 [arXiv:hep-ph/0708.4054]; Z. Abidin \& C. E. Carlson, Gravitational form factors of vector mesons in an AdS/QCD model, Phys. Rev. D77 (2008) 095007 [arXiv:hepph/0801.3839]; Gravitational Form Factors in the Axial Sector from an AdS/QCD Model, Phys. Rev. D77 (2008) 115021 [arXiv:hep-ph/0804.0214]; G. Panico \& A. Wulzer, Nucleon Form Factors from 5D Skyrmions [arXiv:hep-ph/0811.2211].

[14] A. Krikun, On two-point correlation functions in AdS/QCD, Phys. Rev. D77 (2008) 126014 [arXiv:hep-th/0801.4215].

[15] C. A. Ballon Bayona, H. Boschi-Filho, N. R. F. Braga, Deep inelastic structure functions from supergravity at small x, JHEP 0810 (2008) 088 [arXivhep-th/:0712.3530].

[16] E. Katz \& M. D. Schwartz, An Eta primer: Solving the U(1) problem with AdS/QCD, JHEP 0708 (2007) 077 [arXiv:hep-ph/0705.0534]; Y. Kim \& H. K. Lee, Consequences of the partial restoration of chiral symmetry in AdS/QCD, Phys. Rev. D77 (2008) 096011 [arXiv:hep-ph/0802.2409].

[17] A. Karch, E. Katz, D.T. Son \& M.A. Stephanov, Linear confinement and AdS/QCD, Phys. Rev. D74 (2006) 015005 [arXiv:hep-ph/0602229].

[18] N. Evans \& A. Tedder, Perfecting the Ultra-violet of Holographic Descriptions of QCD, Phys. Lett. B642 (2006) 546 [arXiv:hep-ph/0609112]; P. Colangelo, F. De Fazio, F. Jugeau \& S. Nicotri, On the light glueball spectrum in a holographic description of QCD, Phys. Lett. B652 (2007) 73 [arxiv:hep-ph/0703316]; Investigating AdS/QCD duality through scalar glueball correlators [arXiv:hep-ph/0711.4747]; F. Jugeau, Holographic description of glueballs in a deformed AdS-dilaton background, AIP Conf. Proc. 964 (2007) 151 [arXiv:hep-ph/0709.1093]; Y. Kim, J.-P. Lee \& S. H. Lee, Heavy quarkonium in a holographic QCD model, Phys. Rev. D75 (2007) 114008 [arXiv:hep-ph/0703172]; H. R. Grigoryan \& A. V. Radyushkin, Structure of vector mesons in holographic model with linear confinement, Phys. Rev. D76 (2007) 095007 [arXiv:hep-ph/0706.1543]; T. Huang \& F. Zuo, Couplings of the Rho Meson in a Holographic dual of QCD with Regge Trajectories, Eur. Phys. J. C56 (2008) 75 [arXiv:hep-ph/0708.0936]; C. A. Ballon Bayona, H. Boschi-Filho, N. R.F. Braga, Deep inelastic scattering from gauge string duality in the soft wall model, JHEP 0803 (2008) 064 [arXiv:hep-th/0711.0221]; J. Erdmenger, N. Evans, I. Kirsch \& E. Threlfall, Mesons in Gauge/Gravity Duals - A Review, Eur. Phys. J. A35 (2008) 81 [arXiv:hep-th/0711.4467]; H. J. Kwee \& R. F. Lebed, Pion Form Factor in Improved Holographic QCD Backgrounds, Phys. Rev. D77 (2008) 115007 [arXiv:hep- 
ph/0712.1811]; B. Batell \& T. Gherghetta, Dynamical Soft Wall AdS/QCD, Phys. Rev. D78 (2008) 026002 [arXiv:hep-ph/0801.4383]; B. Batell, T. Gherghetta \& D. Sword, The Soft-Wall Standard Model [arXiv:hep-ph/0808.3977]; A. Vega \& I. Schmidt, Scalar hadrons in $A d S_{5} \times S^{5}$, Phys. Rev. D78 (2008) 017703 [arXiv:hep$\mathrm{ph} / 0806.2267]$.

[19] P. Colangelo, F. De Fazio, F. Giannuzzi, F. Jugeau \& S. Nicotri, Light scalar mesons in the soft-wall model of AdS/QCD, Phys. Rev. D78 (2008) 055009 [arXiv:hep$\mathrm{ph} / 0807.1054]$.

[20] M. Peter, The static quark-anti-quark potential in QCD to three loops, Phys. Rev. Lett. 78 (1997) 602 [arXiv:hep-ph/9610209], The Static potential in QCD: A Full two loop calculation, Nucl. Phys. B501 (1997) 471 [arXiv:hep-ph/9702245]; Y. Schroder, The Static potential in QCD to two loops, Phys. Lett. B447 (1999) 321 [arXiv:hep$\mathrm{ph} / 9812205]$.

[21] E. Eichten, K. Gottfried, T. Kinoshita, K. D. Lane \& T.-M. Yan, Charmonium: The Model, Phys. Rev. D17 (1978) 3090 [Erratum-ibid. D21 (1980) 313].

[22] W. Lucha, F. F. Schoberl \& D. Gromes, Bound states of quarks, Phys. Rept. 200 (1991) 127.

[23] G. S. Bali, QCD forces and heavy quark bound states, Phys. Rept. 343 (2001) 1 [arXiv:hep-ph/0001312].

[24] L. S. Brown \& W. I. Weisberger, Remarks on the static potential in quantum chromdynamics, Phys. Rev. D20 (1979) 3239.

[25] K. G. Wilson, Confinement of quarks, Phys. Rev. D10 (1974) 2445.

[26] D. LaCourse \& M. G. Olsson, String potential model: Spinless quarks, Phys. Rev. D39 (1989) 2751; C. Olson, M. G. Olsson \& K. Williams, QCD, relativistic fulx tubes and potential models, Phys. Rev. D45 (1992) 4307; M. G. Olsson \& K. Williams, QCD and the relativistic flux tube with fermionic ends, Phys. Rev. D48 (1993) 417.

[27] S. W. Hawking \& D. N. Page, Thermodynamics Of Black Holes In Anti-de Sitter Space, Commun. Math. Phys. 87 (1983) 577.

[28] S. S. Gubser, I. R. Klebanov \& A. A. Tseytlin, Coupling Constant Dependence in the Thermodynamics of $N=4$ Supersymmetric Yang-Mills Theory, [arXiv:hepth/9805156]. 
[29] A. Brandhuber, N. Itzhaki, J. Sonnenschein \& S. Yankielowicz, Wilson loops in the large $N$ limit at finite temperature, Phys. Lett. B434 (1998) 36 [arXiv:hepth/9803137].

[30] S.-J. Rey, S. Theisen \& J.-T. Yee, Wilson-Polyakov loop at finite temperature in large $N$ gauge theory and anti-de Sitter supergravity, Nucl. Phys. B527 (1998) 171 [arXiv:hep-th/9803135].

[31] J. Greensite \& P. Olesen, Remarks on the heavy quark potential in the supergravity approach, JHEP 9808 (1998) 009 [arXiv:hep-th/9806235].

[32] A. Brandhuber, N. Itzhaki, J. Sonnenschein \& S. Yankielowicz, Wilson loops, confinement, and phase transitions in large $N$ gauge theories from supergravity, JHEP 9806 (1998) 001 [arXiv:hep-th/9803263].

[33] M. J. Teper, SU(N) gauge theories in (2+1)-dimensions, Phys. Rev. D59 (1999) 014512 [arXiv:hep-lat/9804008].

[34] M. Luscher, K. Symanzik \& P. Weisz, Anomalies of the Free Loop Wave Equation in the WKB Approximation, Nucl. Phys. B173 (1980) 365.

[35] D. J. Gross \& H. Ooguri, Aspects of large $N$ gauge theory dynamics as seen by string theory, Phys. Rev. D58 (1998) 106002 [arXiv:hep-th/9805129].

[36] J. Greensite \& P. Olesen, World sheet fluctuations and the heavy quark potential in the AdS/CFT approach, JHEP 9904 (1999) 001 [arXiv:hep-th/9901057].

[37] N. Itzhaki, J. M. Maldacena, J. Sonnenschein \& S. Yankielowicz, Supergravity and the large $N$ limit of theories with sixteen supercharges, Phys. Rev. D58 (1998) 046004 [arXiv:hep-th/9802042].

[38] O. Andreev, 1/q2 corrections and gauge/string duality, Phys. Rev. D73 (2006) 107901 [arXiv:hep-th/0603170].

[39] O. Andreev \& V. I. Zakharov, Heavy-quark potentials and AdS/QCD, Phys. Rev. D74 (2006) 025023 [arXiv:hep-ph/0604204].

[40] O. Andreev \& V. I. Zakharov, The Spatial String Tension, Thermal Phase Transition, and $A d S / Q C D$, Phys. Lett. B645 (2007) 437 [arXiv:hep-ph/0607026].

[41] O. Andreev \& V. I. Zakharov, On Heavy-Quark Free Energies, Entropies, Polyakov Loop, and AdS/QCD, JHEP 0704 (2007) 100 [arXiv:hep-ph/0611304]. 
[42] O. Andreev, Some Multi-Quark Potentials, Pseudo-Potentials and AdS/QCD, Phys. Rev. D78 (2008) 065007 [arXiv:hep-ph/0804.4756].

[43] J. P. Shock, F. Wu, Y.-L. Wu, Z.-F. Xie, AdS/QCD Phenomenological Models from a Back-Reacted Geometry, JHEP 0703 (2007) 064 [arXiv:hep-ph/0611227].

[44] C D White, The Cornell potential from general geometries in AdS/QCD, Phys. Lett. B652 (2007) 79 [arXiv:hep-ph/0701157].

[45] L. Randall \& R. Sundrum, A Large mass hierarchy from a small extra dimension, Phys. Rev. Lett. 83 (1999) 3370 [arXiv:hep-ph/9905221]; An Alternative to compactification, Phys. Rev. Lett. 83 (1999) 4690 [arXiv:hep-th/9906064].

[46] H. Boschi-Filho, N. R.F. Braga \& C. N. Ferreira, Static strings in RandallSundrum scenarios and the quark anti-quark potential, Phys. Rev. D73 (2006) 106006 [arXiv:hep-th/0512295]; Erratum, Phys. Rev. D74 (2006) 089903 [arXiv:hepth/0610131].

[47] H. Boschi-Filho, N. R.F. Braga \& C. N. Ferreira, Heavy quark potential at finite temperature from gauge/string duality, Phys. Rev. D74 (2006) 086001 [arXiv:hepth/0607038].

[48] U. Gursoy, E. Kiritsis \& F. Nitti, Exploring improved holographic theories for QCD: Part II, JHEP 0802 (2008) 019 [arXiv:hep-th/0707.1349].

[49] E. Witten, Baryons and branes in Anti-de-Sitter space, JHEP 9807 (1998) 006 [arXiv:hep-th/9805112].

[50] A. Brandhuber, N. Itzhaki, J. Sonnenschein \& S. Yankielowicz, Baryons from Supergravity, JHEP 9807 (1998) 020 [arXiv:hep-th/9806158].

[51] S. Mandelstam, Feynman rules for electromagnetic and Yang-Mills fields from the gauge-independent field-theoretic formalism, Phys. Rev. 175 (1968) 1580.

[52] A. M. Polyakov, Gauge fields as rings of glue, Nucl. Phys. B164 (1979) 171.

[53] Yu. M. Makeenko, Large- $N$ gauge theories, Lectures given at NATO Advanced Study Institute on Quantum Geometry, Akureyri, Iceland (1999) [arXiv:hep-th/0001047].

[54] F. Jugeau \& H. Sazdjian, Bound-state equation in the Wilson loop approach with minimal surfaces, Nucl. Phys. B670 (2003) 221 [arXiv:hep-ph/0305021].

[55] A. A. Migdal, Loop equations and 1/N expansion, Phys. Rep. 102 (1983) 199. 
[56] A. M. Polyakov, String representations and hidden symmetries for gauge fields, Phys. Lett. 82 (1979) 255.

[57] T. Nambu, QCD and the string model, Phys. Lett. B80 (1979) 372.

[58] J. L. Gervais \& A. Neveu, The quantum dual string wave functional in Yang-Mills theories, Phys. Lett. B80 (1979) 255.

[59] S. Mandelstam, Charge-monopole duality and the phases of non-Abelian gauge theories, Phys. Rev. D19 (1979) 2391.

[60] R. Giles, Reconstruction of gauge potentials from Wilson loops, Phys. Rev. D24 (1981) 2160.

[61] R. A. Brandt, A. Gocksch, F. Neri \& M.-A. Sato, Loop space, Phys. Rev. D26 (1982) 3611.

[62] G. 't Hooft, A planar diagram theory for strong interactions, Nucl. Phys. B72 (1974) 461.

[63] G. 't Hooft, A two-dimensional model for mesons, Nucl. Phys. B75 (1974) 461.

[64] V. S. Dotsenko \& S. N. Vergeles, Renormalizability of phase factors in non-abelian gauge theory, Nucl. Phys. B169 (1980) 527.

[65] R. A. Brandt, F. Neri \& M.-A. Sato, Renormalization of loop functions for all loops, Phys. Rev. D24 (1981) 879.

[66] Y. Frishman \& J. Sonnenschein, Bosonization and QCD in two dimensions, Phys. Rept. 223 (1993) 309.

[67] E. Abdalla \& M.C.B. Abdalla, Updating $Q C D_{2}$, Phys. Rept. 265 (1996) 253.

[68] V. A. Kazakov \& I. K. Kostov, Non-linear strings in two-dimensional $U(\infty)$ gauge theory, Nucl. Phys. B176 (1980) 199.

[69] V. A. Kazakov, Wilson loop average for an arbitrary contour in two-dimensional $U(N)$ gauge theory, Nucl. Phys. B179 (1981) 283. 